Aus der Abteilung Allgemeinmedizin

(Prof. Dr. med. M. M. Kochen, MPH, FRCGP)

im Zentrum Innere Medizin

der Medizinischen Fakultät der Universität Göttingen

\title{
Leitlinienadhärenz hausärztlicher Pharmakotherapie der chronischen Herzinsuffizienz
}

\begin{abstract}
Einfluss von Diagnosesicherung und Therapieempfehlungen an der stationär-ambulanten Schnittstelle
\end{abstract}

\author{
INAUGURAL - DISSERTATION \\ zur Erlangung des Doktorgrades \\ der Medizinischen Fakultät \\ der Georg-August-Universität zu Göttingen
}

\author{
vorgelegt von \\ Christian Pfeil
}

aus

Rostock

Göttingen 2006 
D e k a n:

Prof. Dr. med. C. Frömmel

I. Berichterstatter: Prof. Dr. med. M. M. Kochen, MPH, FRCGP

II. Berichterstatter/in:

III. Berichterstatterlin:

Tag der mündlichen Prüfung: 


\section{Inhalt}

1 EINLEITUNG

2 STAND DER FORSCHUNG. 3

2.1 Grundlagen der chronischen Herzinsuffizienz ................................................3

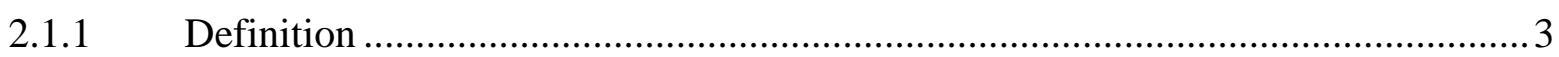

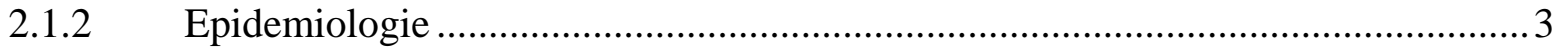

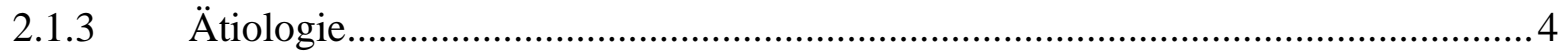

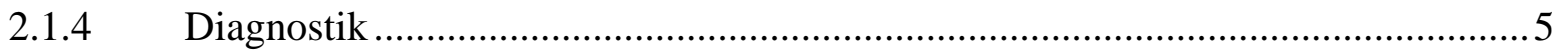

2.2 Therapeutische Ansätze in der Behandlung der Herzinsuffizienz...........................7

2.2.1 Therapeutische Ansätze nach Evidenzkriterien ...............................................

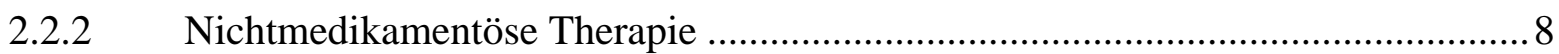

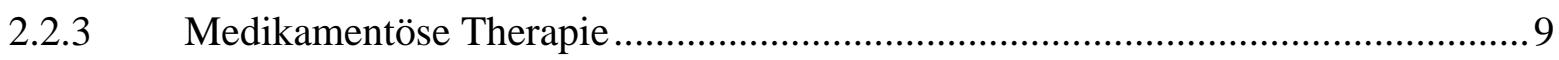

2.3 Hausärztliche Versorgung von Patienten mit Herzinsuffizienz ...........................14

2.3.1 Bedeutung und Besonderheiten hausärztlicher Versorgung ................................ 14

2.3.2 Der Hausarzt im Behandlungskontinuum .................................................... 15

3 FRAGESTELLUNG UND ZIELSETZUNG DER UNTERSUCHUNG ............17

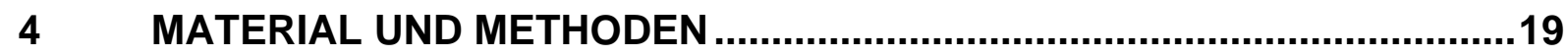

4.1 Methodische Anlage der Untersuchung .........................................................19

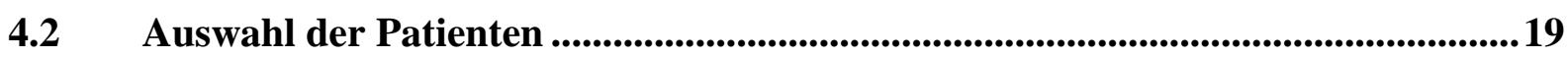

4.2.1 Patienten der poststationären Gruppe ......................................................... 19

4.2.2 Patienten der Kontrollgruppe ..................................................................... 21

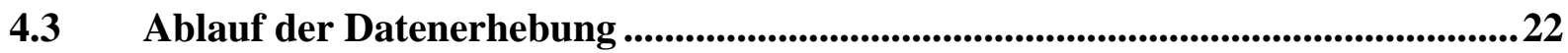

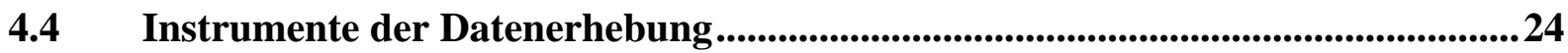

4.5 Beurteilung der Medikation im Verlauf nach Leitlinienadhärenz ........................25

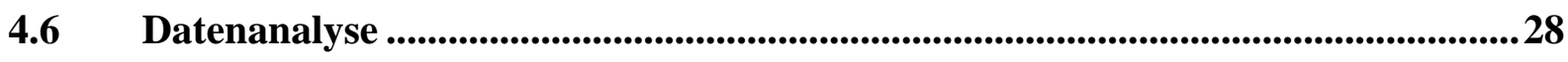


4.7 Datenschutz und Genehmigung durch die Ethik-Kommission.

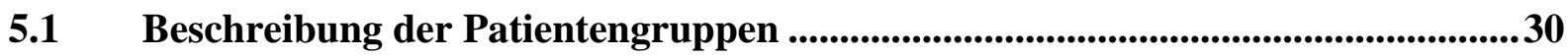

5.1.1 Patienten der poststationären Gruppe ........................................................ 30

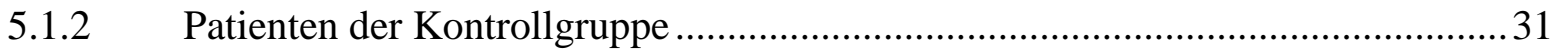

5.2 Vergleich der Patientengruppen bezüglich entscheidender soziodemographischer und klinischer Daten 31

5.3 Überblick über die kardiovaskuläre Medikation im zeitlichen Verlauf ..............33

5.3.1 Verwendete Einzelstoffe der kardiovaskulären Medikation .................................33

5.3.2 Baseline-Erhebung: Verschreibungshäufigkeit in beiden Gruppen......................35

5.3.3 Follow-Up-Erhebung: Verschreibungshäufigkeit in beiden Gruppen ...................36

5.4 Verordnungshäufigkeiten von Medikamenten mit Verbesserung der Prognose (Evidenz-A-Medikamente)..............................................................................38

5.5 Dosierungsbereiche mit Blick auf Zieldosierungen aus Empfehlungen der Leitlinien.........................................................................................................

5.6 Hospitalisierung und Krankheitsverlauf im Untersuchungszeitraum .................43

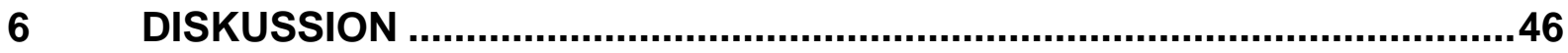

6.1 Zielsetzung und Hauptergebnisse der Studie..................................................46

6.2 Leitlinienadhärenz der Pharmakotherapie an der stationär - ambulanten Schnittstelle ..................................................................................................................4 47

6.2.1 Verordnungsraten kardiovaskulärer Medikation im zeitlichen Verlauf ...............47

6.2.2 Mögliche Gründe der mangelnden Leitlinienadhärenz......................................50

6.2.3 Dosierung kardiovaskulärer Medikation im zeitlichen Verlauf...........................55

6.3 Methodische Diskussion der Ergebnisse .....................................................60

6.3.1 Repräsentativität und Vergleichbarkeit der untersuchten Patientengruppen ........60

6.3.2 Güte der eingesetzten Erhebungsinstrumente .................................................61

6.3.3 Limitationen des Untersuchungsdesigns.....................................................63 
6.4 Bewertung von Leitlinien-Empfehlungen als „Goldstandard“ in der

Pharmakotherapie primärärztlich versorgter Patienten. .64

6.5 Schlussfolgerung und Ausblick 66

7 ZUSAMMENFASSUNG .68

8 LITERATURVERZEICHNIS .70

9 ANHANG 78

A.1 Vergleich der Verordnungen mit Daten anderer Studien ...................................... 78

A.2 Vergleich von empfohlenen Zieldosen und maximalen Tagesdosen der verwendeten Medikamente 78

A.3 Vergleich der durchschnittlichen Arzneimittelkosten für ACE-Hemmer und BetaBlocker (aus: Rote Liste Service GmbH, Stand: 27.07.2006) 79

A.4 Flussdiagramm zur Pharmakotherapie nach klinischen Gesichtspunkten (aus: Leitliniengruppe Hessen: Hausärztliche Leitlinie Chronische Herzinsuffizienz 2003, S.14) .80

A.5 Aktuelle Empfehlungen zur Therapie der Herzinsuffizienz.................................. 81

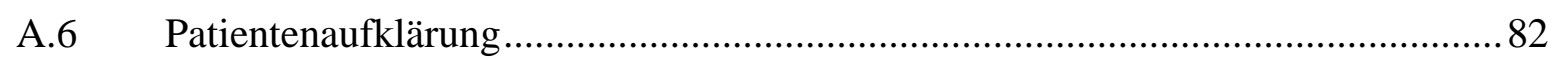

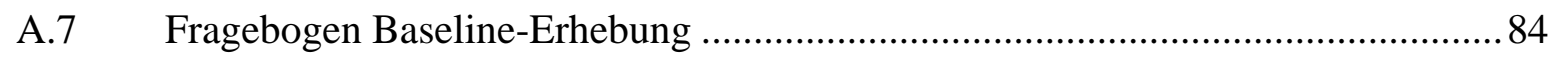

A.8 Telefoninterview (exemplarisch aus der Follow-Up-Untersuchung).....................87

A.9 Fragebogen (Auszug aus der Follow-Up-Untersuchung) ....................................97

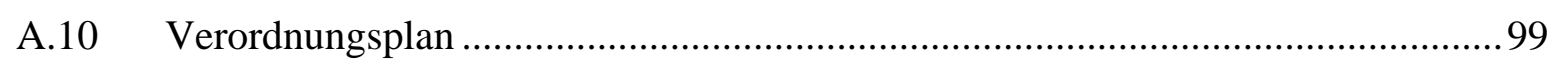




\section{Tabellen- und Abbildungsverzeichnis}

Tabelle 1 : Ursachen der Herzinsuffizienz (modifiziert nach: Leitliniengruppe Hessen 2003, S.26)

Tabelle 2 : Einteilung der Schwere der Herzinsuffizienz nach klinischen Kriterien (modifiziert nach: Alexander et al. 1999, S.1070) 6

Tabelle 3 : Behandlungsziele (Deutsche Gesellschaft für Kardiologie 2001, S.3)..................7

Tabelle 4 : Evidenz-Kriterien (Leitliniengruppe Hessen 2003, S.4) ....................................... 8

Tabelle 5 : Allgemeine Maßnahmen zur Behandlung der Herzinsuffizienz (Auswahl nach: Leitliniengruppe Hessen 2003, S.12) ................................................................. 9

Tabelle 6 : Medikamente mit Verbesserung der Prognose (Evidenz A) .................................10

Tabelle 7 : Referenzleitlinien zum inhaltlichen Vergleich ...................................................27

Tabelle 8 : Kohorten im Verlauf der Datenerhebung .............................................................30

Tabelle 9 : Soziodemographische und klinische Charakteristika der poststationären und der Kontrollgruppe zur Baseline-Erhebung ...............................................................32

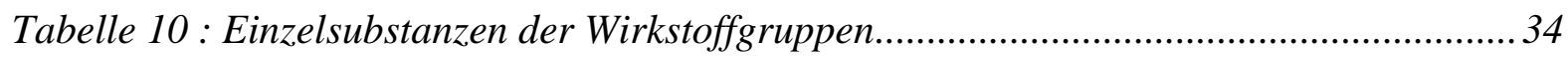

Tabelle 11 : Verordnung von Medikamenten zum Zeitpunkt Null (Baseline-Erhebung) und nach 12 Monaten (Follow-Up-Erhebung) in beiden Gruppen ............................38

Tabelle 12 : Verschreibung von Medikamenten mit Verbesserung der Prognose ....................39

Tabelle 13 : Gründe für den Therapieabbruch mit ACE-Inhibitoren in der poststationären Kohorte $(n=15$ von 43$)$.

Tabelle 14 : Dosierung von ACE-Inhibitoren und Betaadrenozeptor-Antagonisten in Bezug auf empfohlene Zieldosierungen

Tabelle 15 : Krankenhausaufenthalte der Patienten im Untersuchungszeitraum von zwölf Monaten 


\section{$1 \quad$ Einleitung}

Der große Arzt und Wissenschaftler Professor Bernhard Lown hatte nicht nur als Kardiologe mit seiner Klassifikation der ventrikulären Extrasystolen und seiner Erfindung der Defibrillation Weltruhm erlangt. Durch sein weitgefächertes soziales Engagement erhielt er für die Gründung der Organisation „International Physicians for the Prevention of Nuclear War“ (IPPNW) und der damit verbundenen Aufklärung und Meinungsbildung in der Bevölkerung gemeinsam mit seinem russischen Kollegen Chazow 1985 in Oslo den Friedensnobelpreis. Als herausragender Lehrer beschreibt er in seinem Buch „Die verlorene Kunst des Heilens“ (Lown 2002) eine seiner Patientinnen zu Beginn seiner Laufbahn im Jahre 1950 wie folgt: „... Frau M. befand sich im Zustand des Herzversagens mit Flüssigkeitsansammlung in den Lungen und massiv ödematös angeschwollenen Beinen. Das Einatmen von Sauerstoff vermochte ihre rasche und angestrengte Atmung nicht zu bessern. Eine Ursache für die Lungenstauung war ihr außerordentlich schneller Puls - ein Stakkato von hundertneunzig Schlägen pro Minute. Selbst ein gesundes Herz dekompensiert, wenn es dreimal so schnell wie normal schlagen muss. Frau M.'s Herz war aber bereits durch ein in der Kindheit durchgemachtes rheumatisches Fieber schwer geschädigt... . Am nächsten Morgen raste ich auf die Station, um nach Frau M. zu schauen, aber ihr Bett war leer. Sie war während der Nacht gestorben... ." (Lown 2002, S.136).

In dieser Zeit sah man sich größtenteils noch außerstande, derartig schwerkranke Patienten längerfristig erfolgreich zu behandeln. Eine Pharmakotherapie mit Diuretika und Digitalispräparaten konnte jedoch schon damals die belastenden Symptome lindern und dem Patienten Erleichterung verschaffen. Bernhard Lown berichtet seinerzeit von den „wahrhaften Wunderdrogen“: „... Exzessive Flüssigkeit, die in die Körperhöhlen abgeschieden wird und sich in geschwollenen Knöcheln manifestiert, wird ausgeschieden. Durch die Flüssigkeitseinlagerung bedingtes krankhaftes Übergewicht wird rasch und umfassend reduziert. Die Atmung wird verbessert. Das Rasen des Pulses wird gezügelt. Der Patient kann ohne zu keuchen herumlaufen. Das Sichausstrecken im Bett wird nicht länger von erstickendem Husten begleitet. Erstmals seit Wochen kann der Patient eine Nacht durchschlafen. Die lähmende Müdigkeit, welche die kleinste Anstrengung zur unüberwindlichen Aufgabe werden lässt, verschwindet... ." (Lown 2002, S.136). 
Seit den ersten Erfahrungen Lowns auf diesem Gebiet ist ein halbes Jahrhundert vergangen. Seither hat es in der Medizin besonders in der Pharmakotherapie enorme Fortschritte gegeben. Dennoch bleibt die chronische Herzinsuffizienz auch im 21. Jahrhundert eine schwere und lebensbedrohliche Erkrankung. Neben der Verbesserung der symptomatischen und Akuttherapie zielen die Behandlungskonzepte heute außerdem auf eine Verbesserung der Langzeitprognose ab. Aber wie gehen wir mit unseren neuen Möglichkeiten um? Bedeutet der Fortschritt der Medizin auch einen Vorteil für jeden einzelnen Patienten? Diese und andere Fragen zur gegenwärtigen Versorgung von Patienten mit Herzinsuffizienz besonders im hausärztlichen Bereich sowie mögliche Antworten werden in der vorliegenden Arbeit aufgegriffen. 


\section{$2 \quad$ Stand der Forschung}

\subsection{Grundlagen der chronischen Herzinsuffizienz}

\subsubsection{Definition}

Als Herzinsuffizienz (engl.: [congestive] heart failure, CHF) wird die Unfähigkeit des Herzens, das vom Organismus benötigte Herzzeitvolumen bei normalem enddiastolischen Ventrikeldruck zu fördern, verstanden (Alexander et al. 1999). Die Weltgesundheitsorganisation (WHO) definiert Herzinsuffizienz als die verminderte körperliche Belastbarkeit aufgrund einer ventrikulären Funktionsstörung (McMurray 1996). Die Herzinsuffizienz ist dabei aber überwiegend als ein klinisches Symptom unterschiedlichster Ätiologie und weniger als eigenständige Erkrankung zu verstehen. Damit liegt das Hauptaugenmerk noch vor einer Therapie der chronischen Herzinsuffizienz selbst auch auf der primären und sekundären Prävention von Risikofaktoren und ursächlichen Erkrankungen.

\subsubsection{Epidemiologie}

Die chronische Herzinsuffizienz stellt ein bedeutendes und wachsendes Gesundheitsproblem in der westlichen Welt dar. Die Inzidenz steigt exponentiell mit zunehmendem Alter und beträgt zirka $0,2 \%$ bei den 54 - bis 64 -Jährigen und bereits $1 \%$ bei den 75- bis 84-Jährigen. Männer erkranken häufiger als Frauen (1\% der Männer und $0,6 \%$ der Frauen der 75 - bis $84-J a ̈ h r i g e n)$. Die Prävalenz zeigt sich ebenfalls altersabhängig mit 3\% in der sechsten Lebensdekade bis hin zu 10\% im achtzigsten Lebensjahr (Murray-Thomas und Cowie 2003). Laut den Autoren der Leitlinie "Chronische Herzinsuffizienz" der Deutschen Gesellschaft für Allgemeinmedizin und Familienmedizin (DEGAM) sind in Deutschland derzeit bis zu 1,6 Millionen Menschen betroffen (Kompetenznetz Herzinsuffizienz 2006).

Bedingt vor allem durch gehäufte und verlängerte Krankenhausaufenthalte erfordert die Behandlung der Herzinsuffizienz schon heute etwa 1-2\% der Ausgaben der Gesundheitssysteme westlicher Länder (Komajda et al. 2001). Aufgrund der zuneh- 
menden Überalterung der Bevölkerung sind steigende Neuerkrankungsraten und damit höhere Ausgaben noch zu erwarten.

Die Prognose der Herzinsuffizienz ist schlecht. Mit einer mittleren Überlebenszeit von 1,7 Jahren bei Männern und 3,2 Jahren bei Frauen sowie einer 5-JahresÜberlebensrate von $25 \%$ bei Männern und $38 \%$ bei Frauen ist die Letalität hoch (Ho et al. 1993). Auch heute haben sich trotz aller Bemühungen und neuen Ansätze im Bereich der Pharmakotherapie die 5-Jahres-Überlebensraten mit 50\% über alle Altersgruppen noch nicht zufriedenstellend verbessert (Varela-Roman et al. 2005).

\subsection{3 Ätiologie}

Die Herzinsuffizienz stellt das Endstadium unterschiedlicher Erkrankungen dar (Tabelle 1, S.5). Prinzipiell lassen sich systolische, diastolische, rhythmogene und extrakardiale Störungen als Ursachen unterscheiden. Nach der Framingham-Studie (Ho et al. 1993) liegen in der Hälfte aller Fälle arterielle Hypertonie und koronare Herzkrankheit (KHK) zugrunde. Wilhelmsen et al. (2001) fanden bei 937 Patienten mit Herzinsuffizienz als Ursachen in 58,8\% der Fälle eine KHK mit oder ohne Hypertension und in 20,3\% der Fälle einen Hypertonus allein (zusammen 79,1\%). Erkrankungen wie Diabetes mellitus (4,5\% der Fälle) oder Herzklappenerkrankungen (5,8\% der Fälle) spielten eher eine untergeordnete Rolle. 
Tabelle 1 : Ursachen der Herzinsuffizienz (modifiziert nach: Leitliniengruppe Hessen 2003, S.26)

\begin{tabular}{|c|c|}
\hline Ursachen & Besonderheiten \\
\hline \multirow[t]{2}{*}{ Koronare Herzkrankheit } & Herzinfarkt \\
\hline & Myokardischämie \\
\hline \multicolumn{2}{|l|}{ Hypertonie } \\
\hline \multirow[t]{3}{*}{ Kardiomyopathien } & Dilatative Kardiomyopathie \\
\hline & Hypertrophe/obstruktive Kardiomyopathie \\
\hline & $\begin{array}{l}\text { Restriktive Kardiomyopathie (z. B. Amyloidose, Sarkoido- } \\
\text { se, Hämochromatose) }\end{array}$ \\
\hline \multirow[t]{3}{*}{ Erworbene und angeborene Herzvitien } & Mitralklappenerkrankung \\
\hline & Aortenklappenerkrankung \\
\hline & Vorhofseptum- / Ventrikelseptumdefekt \\
\hline \multirow[t]{2}{*}{ Herzrhythmusstörungen } & Tachykarde Form \\
\hline & Bradykarde Form \\
\hline \multirow[t]{3}{*}{ Toxische und medikamentöse Ursachen } & Alkohol \\
\hline & Drogen \\
\hline & Medikamente \\
\hline \multirow[t]{3}{*}{ "High Output" Herzinsuffizienz } & Anämie \\
\hline & Thyreotoxikose \\
\hline & AV-Fistel \\
\hline \multirow[t]{2}{*}{ Erkrankungen des Perikards } & Perikarderguss \\
\hline & Pericarditis constrictiva \\
\hline \multirow[t]{4}{*}{ Primäre Rechtsherzinsuffizienz } & Pulmonale Hypertonie \\
\hline & Cor pulmonale \\
\hline & Lungenembolie \\
\hline & Trikuspidalinsuffizienz \\
\hline
\end{tabular}

\subsubsection{Diagnostik}

Die Verdachtsdiagnose "Herzinsuffizienz" leitet sich aus einer typischen Anamnese (z.B. Leistungsminderung, Belastungsdyspnoe, nächtlicher Husten, Nykturie) sowie aus dem klinischen Untersuchungsbefund (z.B. Unterschenkelödeme, Einflussstauung, Tachykardie, pathologische Herzgeräusche, pulmonale Rasselgeräusche) ab. Anhand dieser klinischen Parameter kann bereits eine Abschätzung des Schwere- 
grades der Erkrankung nach der Stadieneinteilung der New York Heart Association (NYHA) oder nach den Kriterien der American Heart Association (AHA) erfolgen (Tabelle 2). Im Folgenden muss neben der Abklärung eventueller Grunderkrankungen zunächst eine strukturelle Herzerkrankung zur Sicherung der Diagnose nachgewiesen werden. Hierbei stehen technische Untersuchungen wie Elektrokardiographie, eine Röntgenaufnahme des Thorax in zwei Ebenen sowie Laboruntersuchungen zur Verfügung. Als entscheidende Untersuchung zum Nachweis einer strukturellen Herzerkrankung hat sich die Doppler-Echokardiographie bzw. eine Herzkatheteruntersuchung, speziell des linken Herzens, erwiesen. Hierbei kann neben Wandbewegungsstörungen und Vitien eine kardiale Pumpschwäche mit verminderter Auswurfleistung über die Bestimmung der Ejektionsfraktion (EF) festgestellt werden. Zur Einleitung einer adäquaten und ggf. sogar kausalen Therapie ist eine derartige Diagnostik unabdingbar (European Society of Cardiology 2001, S.7).

Tabelle 2 : Einteilung der Schwere der Herzinsuffizienz nach klinischen Kriterien (modifiziert nach: Alexander et al. 1999, S.1070)

\begin{tabular}{|c|c|}
\hline \multicolumn{2}{|l|}{ NYHA - Stadien } \\
\hline I & Beschwerdefreiheit, normale körperliche Belastung möglich \\
\hline II & Belastungsinsuffizienz, Beschwerden bei stärkerer körperlicher Belastung \\
\hline III & $\begin{array}{l}\text { Beginnende Ruheinsuffizienz, Beschwerden bei leichter körperlicher Belas- } \\
\text { tung }\end{array}$ \\
\hline IV & Manifeste Ruheinsuffizienz, Beschwerden in Ruhe \\
\hline \multicolumn{2}{|l|}{ AHA - Stadien } \\
\hline$A$ & Patienten ohne Symptome, aber mit Risikofaktoren für eine Herzinsuffizienz \\
\hline $\begin{array}{c}\text { B } \\
\text { (entspricht NYHA I) }\end{array}$ & $\begin{array}{l}\text { Keine Symptome einer Herzinsuffizienz, aber Zeichen einer strukturellen } \\
\text { Herzschädigung }\end{array}$ \\
\hline $\begin{array}{c}C \\
\text { (entspricht NYHA II+III) }\end{array}$ & $\begin{array}{l}\text { Strukturelle Herzschäden in Verbindung mit Symptomen einer Herzinsuffi- } \\
\text { zienz }\end{array}$ \\
\hline $\begin{array}{c}D \\
\text { (entspricht NYHA IV) }\end{array}$ & Terminale Herzinsuffizienz \\
\hline
\end{tabular}

NYHA: New York Heart Association, AHA: American Heart Association 


\subsection{Therapeutische Ansätze in der Behandlung der Herzinsuffi- zienz}

\subsubsection{Therapeutische Ansätze nach Evidenzkriterien}

Die intensive Erforschung der pathophysiologischen Mechanismen der chronischen Herzinsuffizienz hat in den letzten Jahren zu entscheidenden Änderungen in den Therapiekonzepten geführt. Durch den Nachweis der Senkung der Letalität durch Angiotensin-Converting-Enzyme-Inhibitoren (ACE-Hemmer), BetaadrenozeptorAntagonisten (Beta-Blocker) und Aldosteron-Antagonisten soll vor allem eine Besserung der schlechten Prognose der Herzinsuffizienz erreicht werden.

Generell orientiert sich die Behandlung der Herzinsuffizienz an den von verschiedenen Organisationen (z.B. Deutsche Gesellschaft für Kardiologie) formulierten Therapiezielen (Tabelle 3). Hierbei steht nicht nur die Verbesserung der Prognose im Vordergrund der Bemühungen. In besonderem Maße soll der betroffene Patient auch allägliche Aufgaben (wie beispielweise die eigene Körperpflege, das Ankleiden, etc.) wieder selbst übernehmen können. Eine eventuell fortschreitende Pflegebedürftigkeit soll möglichst lange hinausgezögert werden.

Tabelle 3 : Behandlungsziele (Deutsche Gesellschaft für Kardiologie 2001, S.3)

\begin{tabular}{lll}
\hline \hline Hauptkriterien & $\bullet$ & Verbesserung der Prognose (Senkung der Letalität) \\
& $\bullet$ & Verbesserung der Symptomatik (Verbesserung der Lebensqualität) \\
Nebenkriterien & - Progressionshemmung \\
& - Senkung der Hospitalisierungsrate \\
& $\bullet \quad$ Verbesserung hämodynamischer Parameter \\
\hline \hline
\end{tabular}

Die nachstehenden Evidenzkriterien (Tabelle 4, S.8) sind von der US Agency for Health Care Policy and Research (AHCPR) des US Department of Health and Human Service entwickelt worden und dienen der evidenzbasierten Bewertung therapeutischer Maßnahmen (AHCPR 1993). In der vorliegenden Arbeit werden im besonderen Maße Maßnahmen der Kategorie „A“ von Bedeutung sein, deren Wirksamkeit durch Metaanalysen von randomisierten kontrollierten klinischen Studien belegt werden konnte (Tabelle 4). 
Tabelle 4 : Evidenz-Kriterien (Leitliniengruppe Hessen 2003, S.4)

\begin{tabular}{ll}
\hline \hline Evidenz A & $\begin{array}{l}\text { Evidenz aufgrund von Metaanalysen randomisierter kontrollierter klinischer Studien. } \\
\text { Evidenz ist zuverlässig belegt. }\end{array}$ \\
Evidenz B & $\begin{array}{l}\text { Evidenz aufgrund von gut angelegten, nicht randomisierten klinischen Studien bzw. } \\
\text { klinischer Erfahrung. Evidenz ist gut belegt. }\end{array}$ \\
Evidenz C & $\begin{array}{l}\text { Keine sicheren Studienergebnisse, die eine günstige oder schädigende Wirkung be- } \\
\text { legen. Eventuell Evidenz aufgrund von Expertenmeinungen, Konsensuskonferenzen, } \\
\text { klinischer Erfahrung anerkannter Autoritäten. }\end{array}$ \\
\hline \hline
\end{tabular}

\subsubsection{Nichtmedikamentöse Therapie}

Grundlegende therapeutische Ansätze zielen zuallererst auf eine kausale Therapie der Grunderkrankung ab. Wird die Diagnose „Herzinsuffizienz“ gestellt, müssen mögliche Ursachen wie arterielle Hypertonie oder KHK abgeklärt und behandelt werden, da bereits allein durch diese Maßnahmen die Schwere der Erkrankung gemindert werden kann. Darüber hinaus bzw. begleitend zu einer spezifischen Pharmakotherapie sollten Allgemeinmaßnahmen eingeleitet werden (Leitliniengruppe Hessen 2003, S.9).

Alle in dieser Arbeit beachteten Leitlinien empfehlen als Verhaltensregeln für den Patienten beispielsweise Gewichtsreduktion, Salz- und Flüssigkeitsrestriktion sowie mäßige körperliche Aktivität (z.B. European Society of Cardiology 2001, S.3). Arbeiten von D'Alto et al. zeigten, dass die Aufklärung des Patienten über seine Erkrankung und die Vermittlung einfacher Verhaltensweisen wie regelmäßige Gewichtskontrollen einen positiven Einfluss auf den Verlauf einer bestehenden Herzinsuffizienz und auf die Re-Hospitalisierungsrate haben (D'Alto et al. 2003). In Tabelle 5 werden die wichtigsten allgemeinen Maßnahmen dargestellt. 
Tabelle 5 : Allgemeine Maßnahmen zur Behandlung der Herzinsuffizienz (Auswahl nach: Leitliniengruppe Hessen 2003, S.12)

\begin{tabular}{ll}
\hline \hline Maßnahmen & Evidenz \\
\hline Gewichtsnormalisierung & $\mathrm{C}$ \\
Salzarme Diät (2-3g/Tag) & $\mathrm{C}$ \\
Flüssigkeitsrestriktion (1-2 L/Tag) & $\mathrm{C}$ \\
Pneumokokkenimpfung & $\mathrm{B}$ \\
Grippeimpfungen & $\mathrm{B}$ \\
Tägliche Gewichtskontrolle unter gleichen Bedingungen & $\mathrm{C}$ \\
Regelmäßige, leichte körperliche Aktivität & $\mathrm{A}$ \\
\hline \hline
\end{tabular}

\subsubsection{Medikamentöse Therapie}

Eine medikamentöse Therapie stellt den entscheidenden Grundpfeiler zur Behandlung der Patienten dar.

Zur Verlangsamung der Progression der Erkrankung und damit zur Senkung der Letalität kommen vor allem die nachweislich letalitätssenkenden Evidenz-AMedikamente (Tabelle 6, S.10) zum Einsatz. Des Weiteren sollen aber auch, wie bereits erwähnt, die für den Patienten besonders belastenden Symptome gemildert und damit die Lebensqualität und die Selbstständigkeit verbessert werden. Hierbei müssen Medikamente auch ohne nachweisliche Senkung der Letalität (z.B. Diuretika) angewendet werden. Die Einleitung einer medikamentösen Therapie sollte nach gestellter Diagnose frühzeitig erfolgen, da nur so schwerwiegende strukturelle Herzerkrankungen (wie z.B. progressive Kardiomyopathie) vermieden werden können. Aus diesem Grund ist eine medikamentöse Therapie sowohl bei asymptomatischen Patienten (NYHA I) zur ausschließlichen Prognoseverbesserung wie auch bei Patienten mit Beschwerden zusätzlich zur Symptom- und Befindlichkeitsbesserung indiziert. Gerade die Behandlung scheinbar gesunder Patienten stellt für Arzt und Patient gleichermaßen ein Problem dar.

Bevorzugt sollten Medikamente mit nachgewiesener Verbesserung der Prognose eingesetzt werden. Hierzu gehören ACE-Hemmer oder alternativ bei Unverträglichkeit AT1-Rezeptor-Antagonisten (AT1-Blocker), die Beta-Blocker Bisoprolol, Metopro- 
lol und Carvedilol sowie der Aldosteron-Antagonist Spironolacton. Tabelle 6 gibt einen Überblick über die für die Behandlung der chronischen Herzinsuffizienz zugelassenen Medikamente mit Verbesserung der Prognose. Des Weiteren sollten die in den Richtlinien empfohlenen Zieldosen angestrebt werden, da aus Letalitätsstudien positiv korrelierte Dosis-Wirkungs-Beziehungen abgeleitet werden konnten.

Weitere Medikamente zur Behandlung der Herzinsuffizienz sind vor allem Diuretika (Thiazide und Schleifendiuretika), aber auch Digitalispräparate. Zwar konnte bisher für diese Präparate keine Verbesserung der Prognose, wohl aber eine Verbesserung der Symptomatik nachgewiesen werden, was ihren Einsatz in der stufenweisen Therapie der Herzinsuffizienz rechtfertigt.

Tabelle 6 : Medikamente mit Verbesserung der Prognose (Evidenz A)

\begin{tabular}{lll}
\hline \hline Stoffklasse & Substanz & Prognosebesserung durch Studien \\
\hline ACE-Hemmer & alle & The SOLVD-Investigators (1991) \\
& & Pfeffer et al. (1992) (Save) \\
AT1-Blocker & Losartan & Pitt et al. (2000) (ELITE-II) \\
Beta-Blocker & Bisoprolol & CIBIS-II-Investigators and Committees (1999) \\
& Metoprolol & MERIT-HF-Study-Group (1999) \\
& Carvedilol & Poole-Wilson et al.(2003) (COMET) \\
Aldosteron-Antagonisten & Spironolacton & Pitt et al. (1999) (Rales) \\
\hline \hline
\end{tabular}

\section{ACE-Hemmer}

Die Therapie mit ACE-Hemmern gilt als Goldstandard in der Behandlung der Herzinsuffizienz (Remme et al. 2004).

Neben zahlreichen anderen zeigten die klinischen Studien von The SOLVDInvestigators (1991) an einem Kollektiv von 2569 herzinsuffizienten Patienten eine signifikant niedrigere Letalität der Enalapril-Gruppe (35,2\%; 452 von 1285 Patienten) als Teilnehmer der Placebo-Gruppe (39,7\%; 510 von 1284 Patienten). Ähnlich positive Effekte konnten im Weiteren für eine große Zahl anderer ACE-Hemmer gezeigt werden, so dass davon ausgegangen wird, dass alle ACE-Hemmer die Letalität der Herzinsuffizienz signifikant senken. Dass umgekehrt die Beendigung einer Therapie mit ACE-Hemmern sich negativ auf den Krankheitsverlauf auswirkt, konnten Pflugfel- 
der et al. (1993) zeigen. Sie untersuchten in einer klinischen placebokontrollierten Studie 224 Patienten mit Herzinsuffizienz NYHA II und III bezüglich der Auswirkungen des Aussetzens der ACE-Hemmer-Therapie (Placebogruppe) in einem Zeitraum von 16 Wochen. Es wurde festgestellt, dass sich NYHA-Klasse und "qualitiy-of-lifescore" in der Placebogruppe deutlich verschlechterten. Zudem mussten im Gegensatz zu Patienten der Verumgruppe, die den Wirkstoff Quinalapril weiter erhielten, mehr Patienten wegen sich stark verschlechternder Herzinsuffizienz (NYHA IV) ausgeschlossen werden ( $n=18$ vs. $n=5$ ). Die Beendigung einer Therapie mit ACEHemmern trägt also zur Verschlechterung der bestehenden Erkrankung bei.

\section{AT1-Blocker}

Für AT1-Blocker als Substanzen ebenfalls mit Wirkung auf das Renin-AngiotensinAldosteron-System wurden Effekte ähnlich denen der ACE-Hemmern, nur mit geringeren Nebenwirkungen (Reizhusten) unterstellt. Heute werden sie aber ausschließlich als Alternative zu ACE-Hemmern bei Unverträglichkeit oder Kontraindikationen angesehen. Vertreter dieser Stoffgruppe können im Vergleich zum Placebo die Belastungstoleranz und Symptomatik herzinsuffizienter Patienten signifikant verbessern. Diese Effekte, wie auch die Senkung der Hospitalisationsrate aufgrund einer dekompensierten Herzinsuffizienz, waren in den NYHA-Stadien II-IV vergleichbar mit einer ACE-Hemmer-Therapie oder einer Kombinationstherapie mit ACE-Hemmern und AT1-Blockern (McKelvie et al. 1999, Pitt et al. 1997).

Eine vergleichende Letalitätsstudie zwischen einem AT1-Blocker (Losartan) und einem ACE-Hemmer (Captopril) bei älteren Patienten (> 60 Jahre) mit systolischer Herzinsuffizienz im Stadium NYHA II-IV (EF $\leq 40 \%$ ) zeigte keinen Unterschied hinsichtlich der Gesamtsterblichkeit, der plötzlichen Herztodesrate oder Reanimationshäufigkeit (Pitt et al. 2000). Therapieabbrüche waren unter AT1-Blockern aufgrund einer niedrigeren Nebenwirkungsrate signifikant seltener als unter ACE-Hemmern. Insgesamt konnte aber keine Überlegenheit der AT1-Blocker gegenüber den ACEHemmern gezeigt werden. Aus diesem Grund wird weiterhin empfohlen, AT1-Blocker ausschließlich bei Unverträglichkeit von ACE-Hemmern zu verordneten. ACEHemmer sollen weiterhin Medikamente der ersten Wahl bleiben. 


\section{Beta-Blocker}

Für Beta-Blocker ist die chronische Herzinsuffizienz eine relativ neue Indikation (Hauptstudien s. Tabelle 6). Laut aktueller pharmakologischer Literatur können sie bei kompensierten Krankheitsstadien sowohl die Prognose, als auch die Symptomatik verbessern (Karow, Lang-Roth 2005, S. 239f.). Im Gegensatz zu den ACEHemmern waren zum Zeitpunkt der vorliegenden Untersuchung prognoseverbessernde Eigenschaften lediglich für Metoprolol, Bisoprolol und Carvedilol gezeigt worden.

Die Wirksamkeit von Metoprolol im Vergleich zu Placebo wurde unter anderem von der MERIT-HF-Study-Group (1999) beschrieben. Untersucht wurden 3391 Patienten mit chronischer Herzinsuffizienz NYHA II-IV (EF<40\%) in einer placebokontrollierten, doppelblinden Studie. Die Verumgruppe $(n=1990)$ erhielt, auftitriert über acht Wochen, 200 mg Metoprolol und wurde im Verlauf eines Jahres hinsichtlich der Mortalität beobachtet. In der Metoprololgruppe wurden eine niedrigere Gesamtmortalität (7,2\% vs. $11,0 \%$ ), weniger plötzliche Todesfälle ( $n=97$ vs. $n=135)$ und weniger Todesfälle aufgrund sich verschlechternder Herzinsuffizienz ( $n=30$ vs. $n=58$ ) registriert. Ähnliche Ergebnisse konnten CIBIS II Investigators and Committees (1999) für den Wirkstoff Bisoprolol liefern. Unter nahezu gleichen Bedingungen wurden 2647 Patienten untersucht, die, mit niedrigen Initialdosen beginnend, $10 \mathrm{mg}$ Bisoprolol pro Tag oder ein Placebo erhielten. Auch hierbei konnten nach gut einjähriger Beobachtung eine geringere Gesamtmortalität, (11,8\% vs. $17,3 \%)$ und eine niedrigere Rate an plötzlichen Todesfällen (3,6\% vs. 6,3\%) beobachtet werden.

Besondere Aufmerksamkeit erlangt seit neuestem der Wirkstoff Carvedilol, ein „Hybridblocker" mit Wirkung auf Alpha- und Betarezeptoren. Poole-Wilson et al. (2003) untersuchten an 1511 Patienten mit Herzinsuffizienz (NYHA II-IV) die Gesamtmortalitäten unter Metoprolol oder Carvedilol gegen Placebo (Beta ${ }_{1}$-kardioselektiver gegen nichtselektiven Beta- und Alpha ${ }_{1}$-Betaadrenozeptor-Antagonist). Nach 58 Monaten zeigte sich für die Carvedilol-Gruppe eine insgesamt niedrigere Gesamtmortalität (34\%; 512 von 1511 Patienten) als für die mit Metoprolol behandelten Patienten (40\%; 600 von 1511 Patienten). Carvedilol konnte also gegenüber Metoprolol die Mortalität weiter signifikant senken.

Aufgrund der Datenlage der erwähnten Studien haben Beta-Blocker mittlerweile einen festen Platz in der Behandlung der kompensierten chronischen Herzinsuffizienz Stadium NYHA II-IV. 


\section{Aldosteron-Antagonisten}

Als letzter Pfeiler der Herzinsuffizienztherapie werden Aldosteron-Antagonisten betrachtet. Entscheidend scheint hierbei nicht der diuretische Effekt, sondern der bremsende Eingriff in das Renin-Angiotensin-Aldosteron-System zu sein (Karow, LangRoth 2005, S. 237f.). Aus diesen theoretischen Überlegungen heraus konnte für den Wirkstoff Spironolacton in weiteren Letalitätsstudien Wirksamkeit in der Behandlung der Herzinsuffizienz gezeigt werden. Pitt et al. (1999) verabreichten insgesamt 1663 Patienten zusätzlich zu ihrer bestehenden Medikation (ACE-Hemmer, Schleifendiuretikum, teilweise Digitalispräparat) $25 \mathrm{mg} / \mathrm{d}$ Spironolacton $(\mathrm{n}=822)$ bzw. ein Placebo $(n=841)$. Nach 24 Monaten kam es in der Verumgruppe zu einer insgesamt niedrigeren Gesamtletalität (35\% vs. 46\%), sowie zu einer um 35\% verminderten Hospitalisierungsrate im Untersuchungszeitraum. Es konnte also gezeigt werden, dass Spironolacton zusätzlich zur Standardtherapie bei schwerer chronischer Herzinsuffizienz die Morbidität und die Letalität weiter senken konnte. Deshalb wird die Gabe von Spironolacton bei fortgeschrittenen Erkrankungsstadien (ab NYHA III) empfohlen.

Durch eine konsequente medikamentöse Therapie der symptomatischen und asymptomatischen Herzinsuffizienz sollen die Progression der Erkrankung, die Hospitalisationsrate und die hohe Letalität gesenkt werden. Durch die Besserung der Symptomatik soll der betroffene Patient wieder an Lebensqualität gewinnen. Die lebenslange Behandlung mit zum Teil erheblichen Nebenwirkungen der Medikamente (z.B. Reizhusten, Hypotonie, Impotenz, Gynäkomastie, etc.) erfordert gleichzeitig eine verantwortungsvolle Betreuung durch den Hausarzt sowie ein solides Arzt-PatientenVerhältnis mit guter Compliance der Patienten. 


\subsection{Hausärztliche Versorgung von Patienten mit Herzinsuffizienz}

\subsubsection{Bedeutung und Besonderheiten hausärztlicher Versorgung}

Typische Symptome einer bestehenden Herzinsuffizienz (z.B. Leistungsschwäche, Dyspnoe, Unterschenkelödeme) - ganz gleich ob als erstmalig auftretende Beschwerden oder als Zeichen einer beginnenden Dekompensation - führen den Patienten zunächst zum Hausarzt. Eine Untersuchung von Saudubray et al. (2005) aus Frankreich zeigt, dass die Prävalenz der chronischen Herzinsuffizienz in der hausärztlichen Praxis hoch ist. In der Befragung stellten 434 Hausärzte Daten von insgesamt 1797 Patienten, älter als 60 Jahre, zur Verfügung. Über Versicherungsdaten wurde anschließend eine Prävalenz der chronischen Herzinsuffizienz von $11,9 \%$ in der hausärztlichen Praxis im Vergleich zu 2,2\% in der Gesamtpopulation der über 60-jährigen berechnet. Bei den über 80-jährigen Patienten der Hausärzte litten mehr als ein Fünftel von ihnen an der Erkrankung. Damit obliegt dem Hausarzt die langjährige Betreuung der überwiegenden Mehrheit der Patienten. Diese Tatsache erlangt umso mehr Bedeutung vor dem Hintergrund der bekannten suboptimalen Versorgung von Patienten mit Herzinsuffizienz im hausärztlichen Bereich.

Zahlreiche Arbeiten zur primärärztlichen Versorgung zeigten enorme Differenzen zwischen evidenzbasierter und empfohlener Therapie und deren Umsetzung in der Praxis. Die „IMPROVEMENT-Studie“ (Cleland et al. 2002) untersuchten 11062 hausärztlich versorgte Patienten mit Herzinsuffizienz aus 15 europäischen Ländern unter anderem hinsichtlich Medikation und Tagesdosen. Dabei wurde festgestellt, dass nur in $60 \%$ der Fälle ACE-Hemmer und nur in 34\% der Fälle Beta-Blocker verschrieben wurden. Die Tagesdosen unterschritten in 50\% der Verschreibungsfälle die empfohlenen Werte. Diese Ergebnisse erhalten noch mehr Gewicht im Vergleich zur Versorgung durch Fachärzte im Krankenhaus. Rutten et al. (2003) verglichen eine Gruppe von 103 Patienten mit Herzinsuffizienz aus 31 hausärztlichen Praxen mit 99 Patienten von kardiologischen Stationen aus neun niederländischen Kliniken hinsichtlich Diagnostik und Therapie der bestehenden Erkrankung. Dabei stellte sich heraus, dass Hausärzte nicht nur weniger diagnostische Maßnahmen wie Thorax-Röntgen (Hausärzte: $51 \%$ vs. Fachärzte: $84 \%)$, EKG (39\% vs. 100\%) und DopplerEchokardiographie (12\% vs. 97\%) veranlassten. Sie verordneten auch weniger der potentiell vorteilhaften Medikamente wie ACE-Hemmer (40\% vs. $76 \%$ ), Beta-Blocker ( $9 \%$ vs. $30 \%$ ), Spironolacton (11\% vs. $32 \%$ ) und AT1-Blocker (6\% vs. $13 \%)$. Zum 
einen scheint also eine allgemeine diagnostische Unsicherheit zu bestehen bzw. fehlen Möglichkeiten, diagnostische Maßnahmen durchzuführen, oder die vorhandenen Möglichkeiten (z.B. Überweisung zum Kardiologen) werden zu wenig genutzt. Zum anderen bestehen Unsicherheiten bezüglich der richtigen Therapie vor allem mit ACE-Hemmern. Toal und Walker (2000) bestätigen nach einer Befragung von 160 Hausärzten, dass diese zwar sehr motiviert waren ACE-Hemmer zu verschreiben, sich jedoch sicherer durch eine zuvor durchgeführte Echokardiographie fühlen würden. Die Autoren fordern deshalb ein Untersuchungs- und Therapieprotokoll, um die diagnostischen und therapeutischen Unsicherheiten seitens der Hausärzte zu beseitigen und so die langfristige Therapie der Patienten zu verbessern.

Die Rolle derartiger Protokolle könnten Leitlinien übernehmen. Leitlinien erfüllen die Forderungen von Toal und Walker (2000), da sie dem Arzt einfache Regeln und Algorithmen, die auf gut dokumentierten, evidenzbasierten Daten beruhen, an die Hand geben. Diese sollen als Entscheidungshilfen dem Hausarzt therapeutische Handlungsempfehlungen geben und ihn bei seinem breitgefächerten und damit schwierigen Versorgungsauftrag unterstützen. Durch regelmäßige Überarbeitung und Neuauflagen bleibt der Arzt „up to date“, ohne kontinuierlich medizinische Fachzeitschriften durcharbeiten zu müssen. Das kostenlose Angebot der Leitlinien im Internet ermöglicht darüber hinaus den einfachen Zugang. Da praktikable Leitlinien gleichermaßen evidenzbasiert und zudem in der Praxis möglichem Standard entsprechen sollten (Tierney et al. 2003), sind in die Erarbeitung unter anderem auch Hausärzte selbst miteinbezogen, um klinische Studien und neue Erkenntnisse für die tägliche Praxis nutzbar zu machen (Leitliniengruppe Hessen 2003, S.2).

\subsubsection{Der Hausarzt im Behandlungskontinuum}

Zusammenfassend muss also - um einen optimalen Behandlungserfolg für Patienten mit Herzinsuffizienz zu erzielen - zum einen die Diagnose gesichert und zum anderen eine evidenzbasierte Therapie eingeleitet werden. Bei der Umsetzung dieser Forderungen können und müssen Spezialisten miteinbezogen werden. Nun liefert aber die alleinige fachärztliche Betreuung offenbar auch keine optimalen Ergebnisse, da der Hausarzt am besten mit der individuellen Situation der Patienten vertraut ist. 
Ausschlaggebend scheint vor allem ein Behandlungskontinuum zu sein, das von der hausärztlichen Vorsorgung bzw. der notfallmäßigen Aufnahme über die Überweisung zum Spezialisten bzw. der Einweisung in ein Krankenhaus und zurück in die allgemeinmedizinische Betreuung reicht. Ahmed et al. (2003) beschreiben für die Betreuung im Behandlungskontinuum im Vergleich zur hausärztlichen einerseits, aber auch zur alleinigen fachärztlichen Behandlung andererseits signifikante Unterschiede in Diagnostik und Therapie. Von 1075 Patienten mit Herzinsuffizienz wurden 32\% sowohl von Hausärzten als auch von Kardiologen gemeinsam betreut. Aus dieser Gruppe wurde bei mehr Patienten (75\%) die linksventrikuläre Ejektionsfraktion (Diagnosesicherung) bestimmt (nur Hausarzt: 36\%, nur Kardiologe: 53\%). Zudem wurden mehr Patienten (71\%) mit ACE-Hemmern (Therapieoptimierung) behandelt (nur Hausarzt, nur Kardiologe: je 54\%). Die beste Versorgung erhielten also Patienten, die im Kontinuum Hausarzt-Facharzt betreut wurden. 


\section{$3 \quad$ Fragestellung und Zielsetzung der Untersuchung}

Aus den Ergebnissen der dargestellten Untersuchungen lassen sich die im Folgenden aufgeführten Eckpunkte und Kernprobleme in der hausärztlichen Versorgung von Patienten mit chronischer Herzinsuffizienz formulieren:

- Die langjährige Betreuung von Patienten mit chronischer Herzinsuffizienz ist mehrheitlich die Domäne des Hausarztes.

- Hausärztlich betreute Patienten erhalten generell weniger Diagnostik und sind mit prognoseverbessernden Medikamenten unterversorgt.

- Durch Spezialisten (in der Klinik) betreute Patienten erhalten standardmäßig Diagnostik und sind wesentlich besser mit prognoseverbessernden Medikamenten versorgt.

- Gute Ergebnisse lassen sich durch eine Betreuung der Patienten im Behandlungskontinuum erzielen.

- Als Diagnostik- und Therapieprotokoll können praxisbezogene und evidenzbasierte Leitlinien den Hausarzt bei der weiterführenden Therapie unterstützen.

Die gefundenen Arbeiten bilden die Situation in der Klinik, unter niedergelassenen Fachärzten sowie im hausärztlichen Bereich ab. Des Weiteren beschäftigten sich die zitierten Studien mit der stationär-ambulanten Schnittstelle besonders zwischen Hausärzten und niedergelassenen Fachkollegen. Jedoch ist wenig bekannt über die Schnittstelle zwischen Fachkollegen der Klinik und Hausarzt und vor allem über die langfristigen Auswirkungen dieser Zusammenarbeit auf die hausärztliche Versorgungsqualität bei Patienten mit chronischer Herzinsuffizienz. In der vorliegenden Untersuchung sollen deshalb folgende Aussagen überprüft werden:

- Die Qualität der stationär-fachärztlichen Entlassungsmedikation von Patienten mit Herzinsuffizienz ist in Bezug auf die Leitlinien höher als die der Medikation ambulant-hausärztlich versorgter Patienten.

- Die Qualität der Medikation poststationärer Patienten nach den Leitlinien ist auch nach zwölf Monaten noch höher als die von Patienten ohne stationären Aufenthalt, und zwar bezüglich 
1. der Verordnungshäufigkeit der in den Leitlinien empfohlenen Wirkstoffe 2. der in den Leitlinien empfohlenen Zieldosierungen.

Die vorliegende Arbeit überprüft, ob die hausärztliche Therapie durch Beseitigung von diagnostischen und therapeutischen Unsicherheiten seitens des Hausarztes optimiert werden kann. 


\section{$4 \quad$ Material und Methoden}

\subsection{Methodische Anlage der Untersuchung}

Bei der vorliegenden Studie handelt es sich um eine Fall-Kontroll-Studie. Das Patientenkollektiv umfasste zwei Kohorten, um die Unterschiede zwischen ausschließlich hausärztlich versorgten Patienten (=Kontrollgruppe) und solchen nach krankheitsspezifischem stationären Aufenthalt (=poststationäre Gruppe) zu untersuchen.

Die Befragungen fanden einmal zu Beginn der Erhebung und nach zwölf Monaten statt, um den langfristigen Einfluss des stationären Aufenthaltes auf die Medikation der poststationären Gruppe zu evaluieren.

\subsection{Auswahl der Patienten}

\subsubsection{Patienten der poststationären Gruppe}

Die Teilnehmer der poststationären Kohorte wurden von internistisch-kardiologischen Stationen des Universitätsklinikum Göttingen rekrutiert. Nach der Auswertung der soziodemographischen Daten wurden dann zu einem späteren Zeitpunkt passende Patienten aus Hausarztpraxen, deren Daten bereits aus einer anderen Studie zur Verfügung standen, als Kontrollgruppe ausgewählt.

Für die Rekrutierung von Patienten für die poststationäre Kohorte wurden nach telefonischer Vorankündigung zunächst die Stationsärzte der kardiologischen Stationen aufgesucht. Gemeinsam mit dem behandelnden Arzt wurden Patienten ausgewählt, die als Haupt- oder Nebendiagnose an einer chronischen Herzinsuffizienz litten. Für die Studie kamen hierbei nur solche Patienten in Frage, bei denen im Sinne einer Sicherung der Diagnose die Bestimmung der Ejektionsfraktion (EF) entweder durch Linksherzkatheter oder durch Echokardiographie erfolgt war. Des Weiteren wurde darum gebeten, nach der Entlassung des Patienten in die hausärztliche Versorgung bzw. in eine Rehabilitationsklinik eine Kopie des Entlassungsbriefes an die Abteilung Allgemeinmedizin zur Auswertung der Entlassungsmedikation (Therapieempfehlungen) zu senden. 
Im Anschluss an die Vorauswahl und nach Einsicht in die Krankenakten wurden die entsprechenden Patienten mit der Diagnose „Herzinsuffizienz“ auf den kardiologischen Stationen aufgesucht und um ihre Teilnahme an der Studie gebeten. In diesem Zusammenhang wurde der Inhalt der Erhebung, der zeitliche Rahmen und die Art und Weise der Befragung vorgestellt und mit den Patienten besprochen.

Erklärte ein Patient seine Bereitschaft zur Teilnahme an der Studie, wurden durch eine kurze Befragung neben weiteren Daten zunächst die Medikation vor dem stationären Aufenthalt sowie etwaige Unverträglichkeiten, Allergien oder Kontraindikationen gegenüber Medikamenten erfasst.

Im zweiten Teil des Dokumentationsbogens (Anhang A.7, S.84) wurden Daten wie Einweisungsdiagnose bzw. bekannte Diagnose einer Herzinsuffizienz sowie beschriebene Symptome einer Herzinsuffizienz aus den Einweisungsscheinen bzw. den Überweisungs- und Verlegungsbriefen entnommen. An dieser Stelle wurde auch erneut nach bekannten Kontraindikationen oder Überempfindlichkeitsreaktionen gegenüber Medikamenten gesucht, um Patienten mit positiver Anamnese von beispielsweise ACE-Hemmer-Kontraindikationen von vornherein zu erkennen.

Im letzten Abschnitt des Fragebogens wurden zur Vervollständigung entscheidende Daten wie EF und aktuelle Medikation aus den Krankenakten übernommen.

Neben der Dokumentation der Angaben durch die Untersucher wurde der Patient gebeten, in einem Fragebogen seine derzeitige Belastbarkeit um den Zeitpunkt der Entlassung selbst zu beurteilen. Im Gespräch mit den Teilnehmern erfolgte abschließend der Hinweis auf den weiteren Fortgang der Studie im Hinblick auf die erneute Konsultation im Verlauf.

Nach Abschluss der Rekrutierung wurden nur Patienten in die Studie aufgenommen, die den Einschlusskriterien entsprachen. Diese beinhalteten eine apparativ (EF $<45 \%$ mittels Echokardiographie oder Linksherzkatheter) und klinisch gesicherte Diagnose einer Herzinsuffizienz (laut Einweisungsschein des Hausarztes oder durch den Stationsarzt) jeder Genese und jeden Schweregrades. Die Teilnehmer mussten das 18. Lebensjahr vollendet und ihr Einverständnis zur Teilnahme an der Studie gegeben haben.

Ausgeschlossen wurden Patienten mit terminalen oder schweren dekompensierten Erkrankungen zum Zeitpunkt der Rekrutierung sowie solche, die nicht in die ambulante Betreuung entlassen wurden. Hierzu zählten Patienten, die beispielsweise zu Operationen innerhalb von maximal sieben Tagen wiederaufgenommen oder verlegt 
wurden, aber nicht solche, an deren Behandlung sich ein Aufenthalt in einer Rehabilitationsklinik anschloss. Ausgeschlossen wurden weiterhin Patienten mit bekannter Aortenstenose und dekompensierter Niereninsuffizienz, da es sich hierbei um relativ häufige Kontraindikationen für ACE-Hemmer handelte. Weitere Ausschlusskriterien waren eine fehlende Einwilligungs- und Kommunikationsfähigkeit, beispielsweise wegen schlechter Deutschkenntnisse oder aufgrund von Aphasien.

\subsubsection{Patienten der Kontrollgruppe}

Für die Kontrollgruppe sollten im Anschluss an die erste, stationär/poststationäre Datenerhebung Patienten mit Herzinsuffizienz gefunden werden, die einerseits den Patienten der poststationären Kohorte in wichtigen Merkmalen entsprachen, die jedoch initial nicht im Krankenhaus behandelt wurden. Auf diese Weise sollte ein aussagekräftiger Vergleich prinzipiell ähnlicher Gruppen mit unterschiedlichen Behandlungsvoraussetzungen möglich gemacht werden.

Im Rahmen des Projekts „Medizinische Versorgung in der Praxis“ (MedViP) der Abteilung Allgemeinmedizin der Universität Göttingen wurden neben der poststationären Kohorte von Patienten mit Herzinsuffizienz, wie oben bereits beschriebenen, auch Praxis-Routinedaten erhoben. Ausgewählte Diagnosen, Verordnungen, Leistungen und Überweisungen wurden rückwirkend über die Behandlungs-Daten-Träger (BDT)-Schnittstelle der Praxis-Software in anonymisierter Form exportiert. Alle persönlichen Patientendaten (z.B. Adressen) wurden durch ein vorgeschaltetes Filterprogramm gelöscht oder pseudonymisiert (Ersetzen von Namen durch Codes). Eine Re-Identifizierung individueller Patienten war nur durch den betreuenden Hausarzt anhand einer speziell gesicherten Code-Liste möglich. In einzelnen Teilprojekten wurden jeweils ergänzende Datenerhebungen durchgeführt, wie beispielsweise Telefoninterviews zum Behandlungsverlauf (Wetzel et al. 2003).

Für ein Teilprojekt zur Verlaufsbeobachtung der hausärztlichen Behandlung von Herzinsuffizienz wurden Patienten mit hausärztlich dokumentierter Herzinsuffizienzdiagnose zufällig aus den BDT-Daten ausgewählt und um ihr Einverständnis zur Teilnahme an der Studie gebeten. Der Kontakt wurde aus Datenschutzgründen über den Hausarzt hergestellt. 
Insgesamt wurden dabei 4120 Herzinsuffizienz-Patienten aus 44 Praxen identifiziert. Im Zeitraum von April 2003 bis Juli 2004 erhielten die Hausärzte Listen mit den Patientennummern und Geburtsdaten, denen anhand der BDT - Daten die Diagnose „Herzinsuffizienz“ zugeordnet worden war. Sie wurden gebeten, die Patienten in ihrem Praxis-Softwaresystem zu re-identifizieren und auf Ausschlusskriterien zu überprüfen.

Ausgeschlossen wurden Patienten bei denen sich die Diagnose Herzinsuffizienz als nicht zutreffend herausstellte (erlebte Anamnese), die terminal krank oder verstorben waren, die nur vertretungsweise die jeweilige Praxis aufgesucht hatten, die verzogen oder pflegebedürftig waren, die an einer malignen Erkrankung litten oder die mangelnde Sprachkenntnisse aufwiesen.

Mithilfe derselben, bereits in der poststationären Kohorte verwendeten Fragebögen (Anhang A.9, S 97-98) bzw. Telefoninterviews (Anhang A.8, S. 87-96) wurden das aktuelle Stadium bzw. die Schwere der Herzinsuffizienz sowie die aktuelle Medikation erfragt und dokumentiert. Zusammen mit den Daten der hausärztlichen Routinedokumentation lagen ausreichend Informationen vor, um aus den teilnehmenden $\mathrm{Pa}$ tienten geeignete Probanden für die Kontrollgruppe zu identifizieren.

Die Parallelisierung der beiden Gruppen erfolgte nach den Parametern Alter, Geschlecht, NYHA-Stadium (Selbstbeurteilung durch den Patienten) und relevante Komorbiditäten (Hypertonie, Diabetes mellitus, Hyperlipidämie, Koronare Herzkrankheit, kompensierte Niereninsuffizienz, chronisch-obstruktive Lungenerkrankung).

\subsection{Ablauf der Datenerhebung}

In der poststationären Gruppe wurde zunächst das NYHA-Stadium mittels eines Fragebogens (Selbstbeurteilung durch den Patienten) bestimmt. Zur endgültigen Diagnosesicherung wurde die durch Linksherzkatheter oder Echokardiographie bestimmte EF aus der Krankenakte übernommen. Im weiteren Verlauf wurden die Entlassungsbriefe der jeweiligen Klinik im Hinblick auf die weiter empfohlene medikamentöse Therapie ausgewertet. Die hier dokumentierte Medikation wurde als Grundlage für die weitere Versorgung durch den Hausarzt angesehen (Therapieempfehlung). 
Besondere Berücksichtigung bei der Erhebung der Entlassungsmedikation fanden vor allem auch die Therapieempfehlungen der Rehabilitationskliniken und Kliniken zur Anschlussheilbehandlung. Da sich bei Klinikaufenthalten mit entsprechenden poststationären Maßnahmen die Entlassungsmedikation teilweise dramatisch änderte, waren gerade diese Empfehlungen als Basis für den Hausarzt und damit als stationär-ambulante Schnittstelle anzusehen.

Nach zwölf Monaten wurden die Teilnehmer erneut in gleicher Weise telefonisch befragt und anschließend um die Bearbeitung und Rücksendung des zugeschickten Fragebogens gebeten. Hierbei wurde besonderes Augenmerk auf die derzeitig aktuelle Medikation und Gründe für eine veränderte Behandlung gelegt, um die hausärztliche Therapie im Vergleich zur Entlassungsmedikation beurteilen zu können. Des Weiteren konnte aus den Angaben der Patienten erneut das aktuelle NYHA-Stadium bestimmt werden. Darüber hinaus wurden Klinikaufenthalte aufgrund kardiologischer Zwischenfälle dokumentiert.

Die Patienten der Kontrollgruppe wurden analog zur poststationären Kohorte sowohl zum Zeitpunkt Null (Entlassungszeitpunkt der poststationären Patienten) als auch erneut nach zwölf Monaten kontaktiert. Die entsprechenden Daten wurden zur Baseline-Erhebung mittels des erwähnten Fragebogens in Verbindung mit einem durch den Patienten auszufüllenden Verordnungsplan erhoben. Die Follow-UpUntersuchung erfolgte per Telefoninterview und Fragebogen. Der gesamte zeitliche und inhaltliche Verlauf ist in Abbildung 1 (S.24) dargestellt. 
Abbildung 1 : Zeitlicher und inhaltlicher Ablauf der Datenerhebung

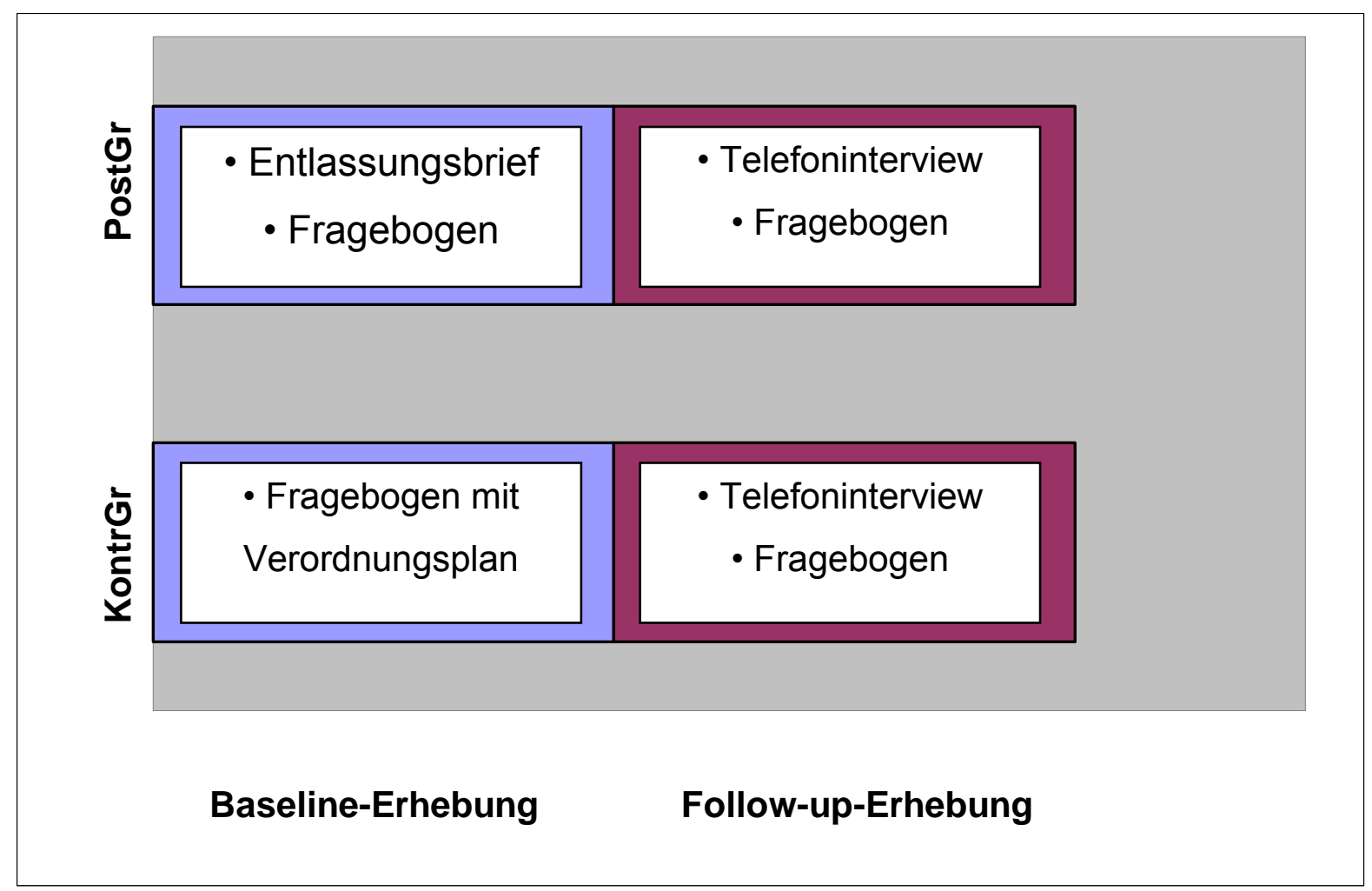

PostGr: Patienten der poststationären Kohorte, KontrGr: Patienten der Kontrollgruppe

\subsection{Instrumente der Datenerhebung}

Zur Erhebung der Daten fanden zwei standardisierte Fragebögen Verwendung (Anhang A.9, S. 97-98; A.8, S.87-96). Einer der Bögen wurde den Patienten zur selbstständigen Beantwortung zugesandt, eine zweite Befragung wurde mithilfe eines telefonischen Interviews durchgeführt.

Die aktuelle Medikation sowie Gründe für veränderte Verordnungen wurden mit dem Patienten persönlich mittels des standardisierten Telefoninterviews besprochen. Hierbei wurde explizit nach Name, Stärke und Dosis des Medikaments gefragt. Die Überprüfung der Vollständigkeit der Angaben erfolgte durch einen Vergleich mit der aus früheren Befragungen (stationärer Aufenthalt bzw. Baseline-Erhebung) erhobenen Vormedikation. Des Weiteren wurden Krankheitsverlauf und Krankenhausaufenthalte im zurückliegenden Untersuchungszeitraum erfasst. Die Patienten wurden zur Dauer des stationären Aufenthalts, nach dem Grund der Einweisung und nach durchgeführten Maßnahmen befragt. 
Die aktuelle Belastbarkeit sowie Beschwerden im Zusammenhang mit der Herzinsuffizienz wurden durch Selbsteinschätzung des Patienten mithilfe des zugeschickten Fragebogens bestimmt. Hierbei musste der Patient im Sinne eines Flussdiagramms Fragen zur Befindlichkeit bei der Ausführung bestimmter Tätigkeiten beantworten. Diese Patientenangaben bildeten die Grundlage für die Klassifikation nach NYHAStadien.

Darüber hinaus erfolgte die Erhebung weiterer Informationen, die in der vorliegenden Arbeit keine Verwendung fanden. Die kompletten Fragebögen können im Anhang eingesehen werden.

Die soziodemographischen Daten, die Komorbiditäten sowie die Entlassungsmedikation der poststationären Kohorte wurden aus den Entlassungsbriefen sowie aus den Krankenakten direkt in die Datenbanken übernommen.

Die Datenerhebung in der Kontrollgruppe erfolgte in gleicher Weise einerseits durch telefonisches Interview und andererseits durch die Beantwortung eines Fragebogens seitens der Patienten. Die soziodemgraphischen Daten wurden der BDT-Datenbank entnommen.

\subsection{Beurteilung der Medikation im Verlauf nach Leitlinienadhä- renz}

Als Referenzleitlinie zur leitliniengerechten medikamentösen Behandlung der chronischen Herzinsuffizienz wurde für die vorliegende Studie die „Hausärztliche Leitlinie“ der Leitliniengruppe Hessen, letzte Auflage 2003, ausgewählt. Diese und Leitlinien zu anderen Erkrankungen können kostenlos unter http://www.leitlinien.de eingesehen werden. Die Wahl fiel auf dieses Werk, da es sich um eine nationale Leitlinie handelte, die von Hausärzten für Hausärzte konzipiert wurde. Weitere Kriterien waren die gute Praktikabilität, die knappe, aber wesentliche Darstellung, meist mithilfe von Tabellen und Schemata sowie die Verdeutlichung der Therapieempfehlungen anhand einfach nachzuvollziehender Entscheidungsbäume. Anwenderfreundlich, aber im Sinne einer Operationalisierung der Leitlinienadhärenz schlecht auswert- und beurteilbar waren die symptomorientierten Empfehlungen zur Gabe der entscheidenden Medikamente gemäß eines dichotomen Entscheidungsbaumes (Anhang A.4, S.80). Auf eine NYHA-stadiengerechte Behandlung nach dem Vorbild internationaler 
Leitlinien wurde hier wegen der besseren Praktikabilität für den behandelnden Arzt verzichtet.

Um für die vorliegende Untersuchung allgemeingültige Standards anwenden zu können, wurde im Vorfeld ein Vergleich mehrerer international anerkannter und viel zitierter Leitlinien bezüglich der grundlegenden Therapieempfehlungen durchgeführt. Hierbei konnten keine wesentlichen Unterschiede gefunden werden, sodass die Inhalte der Leitliniegruppe Hessen (2003) als allgemein akzeptiert und gültig angesehen wurden. Der Vergleich der einzelnen Leitlinien mit der Referenzleitlinie ist in Tabelle 7 (S.27) dargestellt.

In den Empfehlungen der Leitliniengruppe Hessen werden ACE-Hemmer als Medikamente der „ersten Wahl“ (first-line), auch bei asymptomatischer Herzinsuffizienz, angesehen. Sie sind demnach bei nachgewiesener struktureller Herzerkrankung (verminderte EF) in jedem Falle, außer bei Unverträglichkeit oder Kontraindikationen, zu verordnen. Alternativ werden bei ACE-Hemmer-Intoleranz AT1-Blocker empfohlen. Bei persistierenden Symptomen kommen neben Diuretika die Beta-Blocker Metoprolol, Bisoprolol und Carvedilol und im weiteren Verlauf der Aldosteron-Antagonist Spironolacton zum Einsatz. Neben der Verordnung der genannten Wirkstoffe sind die empfohlenen Zieldosen anzustreben (Leitliniengruppe Hessen 2003, S. 13f.). 
Tabelle 7 : Referenzleitlinien zum inhaltlichen Vergleich

\begin{tabular}{|c|c|c|c|c|}
\hline $\begin{array}{l}\text { Bezeichnung, } \\
\text { (Letzte Auflage) }\end{array}$ & $\begin{array}{l}\text { ACC/AHA-Guideline } \\
(\text { USA, 2001) }\end{array}$ & $\begin{array}{l}\text { NICE-Guideline } \\
(U K, 2003)^{6}\end{array}$ & $\begin{array}{l}\text { ESC Guideline } \\
(E U, 2001)^{7}\end{array}$ & Nationale Leitlinien (LI.) \\
\hline Organisationen & $\begin{array}{l}\text { American College of } \\
\text { Cardiology/ American } \\
\text { Heart Association }\end{array}$ & $\begin{array}{l}\text { Guideline by National } \\
\text { Institute of Clinical Ex- } \\
\text { cellence (NICE) }\end{array}$ & European Society of Cardiology & $\begin{array}{l}\text {-LI. der Deutsche Gesellschaft für Kardiologie }{ }^{8} \\
\text { - LI der Arzneimittelkommission d. dt. Ärzteschaft }{ }^{9} \\
\text { - LI. der Leitliniengruppe Hessen }{ }^{10}\end{array}$ \\
\hline $\begin{array}{l}\text { Diagnostische } \\
\text { Kriterien }\end{array}$ & $\begin{array}{l}- \text { AHA-Kriterien } \\
\text {-EF }<40 \% \text { (Echo) }\end{array}$ & -NYHA-Stadien & $\begin{array}{l}\text {-Framingham-Kriterien } \\
\text { •systolische. Dysfkt. (Echo) }\end{array}$ & •NYHA-Stadien, Symptomkontrolle \\
\hline
\end{tabular}

Therapie nach Evidenz A (Letalitätssenkung in Metaanalysen randomisierter kontrollierter klinischer Studien (Tabelle 4, S.8)

\begin{tabular}{|c|c|c|c|c|}
\hline ACE-Hemmer & $\begin{array}{l}\bullet A H A \text { A-D }{ }^{1} \\
\bullet N Y H A \text { I-IV } \\
\bullet \text { kardiovask. Risikopat. }\end{array}$ & $\begin{array}{l}\bullet \text { NYHA I-IV } \\
\bullet Z_{\text {Zieldosierungen }}^{2}\end{array}$ & $\begin{array}{l}\bullet \text { NYHA I-IV } \\
\bullet Z^{2} \text { ieldosierungen }{ }^{2}\end{array}$ & $\begin{array}{l}\text { •NYHA I-IV } \\
\text { •Stufentherapie nach Symptomen } \\
\text { •Zieldosierungen }{ }^{2}\end{array}$ \\
\hline Beta-Blocker & $\begin{array}{l}\text {-ab AHA B }{ }^{1}(\mathrm{NYHA} \text { I) } \\
\text {-Me,Bi,Carve } \\
\text {-Zieldosierungen }{ }^{2}\end{array}$ & $\begin{array}{l}\bullet \text { NYHA I-IV } \\
\bullet \text { Bisoprolol, Carvedilol } \\
\text {-Metoprolol nicht zugel. } \\
\text { •Zieldosierungen }{ }^{2}\end{array}$ & $\begin{array}{l}\text {-NYHA II-IV } \\
\text { •ab NYHA I nur nach Infarkt } \\
\text { •Me,Bi,Carve } \\
\text { •Zieldosierungen }{ }^{2}\end{array}$ & $\begin{array}{l}\text {-alle symptomatischen Pat. mit persistierenden Insuffi- } \\
\text { zienzzeichen unter ACEH } \\
\text { •Me,Bi,Carve }{ }^{3} \\
\text {-Zieldosierungen }{ }^{2}\end{array}$ \\
\hline AT1-Blocker & •bei ACEH-Intoleranz & •nicht zugelassen & •bei ACEH-Intoleranz & •bei ACEH-Intoleranz \\
\hline Spironolacton & $\begin{array}{l}\text { •NYHA IV zusätzlich zu } \\
\text { ACEH }\end{array}$ & $\begin{array}{l}\text { •NYHA III-IV zusätzlich } \\
\text { zu ACEH + Diuretikum }\end{array}$ & $\begin{array}{l}\bullet N Y H A \text { III-IV zusätzlich zu } \\
\text { ACEH + Diuretikum }\end{array}$ & $\begin{array}{l}\text {-alle symptomatischen Pat. mit persistierenden Insuffi- } \\
\text { zienzzeichen unter } \mathrm{ACEH}+\mathrm{BB}+\text { Diuretikum }\end{array}$ \\
\hline
\end{tabular}

LI: Leitlinien, EF: Ejektionsfraktion, Echo: Echokardiographie, ACEH: ACE- ${ }^{5}$ ACC/AHA Guidelines for the Evaluation and Management of Chronic Heart
Hemmer, BB: Beta-Blocker

${ }^{1}$ Stadieneinteilung Herzinsuffizienz nach American Heart Association (Alexander et al. 1999), Stadien A-D failure in the Adult (2001)

${ }^{6}$ National Institute of Clinical Excellence (2003)

${ }^{7}$ European Society of Cardiology (2001)

2 aus Letalitätsstudien abgeleitete und in Leitlinien empfohlene Zieldosierungen (Hochdosistherapie)

${ }^{8}$ Deutsche Gesellschaft für Kardiologie (2001)

${ }^{3}$ Beta-Blocker mit Evidenz A (Metoprolol, Bisoprolol, Carvedilol)

${ }^{9}$ Arzneimittelkommission der deutschen Ärzteschaft (2001)

${ }^{10}$ Leitliniengruppe Hessen (2003) 


\subsection{Datenanalyse}

Zur Auswertung der Arztbriefe sowie der beschriebenen Telefoninterviews und Fragebögen der poststationären Kohorte wurden mithilfe des Programms „MS Access“ Datenbanken erstellt, in die über eine spezifische Eingabemaske alle Daten eingegeben wurden. Hierbei handelte es sich um soziodemographische Patientendaten, um Angaben zur jeweils aktuellen Medikation, zu Begleiterkrankungen, zum Kenntnisstand des eigenen Wissens zur Herzinsuffizienz sowie zum Arzt-PatientenVerhältnis. Die Patientendaten wurden anonymisiert über eine Patienten-ID gespeichert. Die gestellten Diagnosen in Bezug auf die Komorbiditäten wurden nach der ICD-10-Klassifikation (Weltgesundheitsorganisation (WHO) 2005), die verordneten Medikamente gemäß dem ATC-Code (Anatomisch-therapeutisch-chemische Klassifikation für den deutschen Arzneimittelmarkt von Fricke et al. 2004) eingeteilt und kodiert. Es wurden verschiedene Datenbanken für die einzelnen Erhebungszeitpunkte erstellt, die über die Patienten-ID verknüpft waren. Zur Analyse wurden ausgewählte Datensätze in das Statistikprogramm SPSS (Statistical Package for the Social Sciences) für Windows (Version 12.0.1) importiert.

Die Daten der ausgewählten Patienten der Kontrollgruppe wurden der BDTDatenbank entnommen und ergänzend in die zuvor erstellte SPSS-Datenbank integriert.

Die Überprüfung von Unterschiedshypothesen erfolgte mit Hilfe des $\chi^{2}$ Vierfeldertests bzw. des t-Tests für unabhängige Stichproben (zweiseitig getestet bei $\alpha=0,05)$.

\subsection{Datenschutz und Genehmigung durch die Ethik-Kommission}

Patientinnen und Patienten, die für die einzelnen Kohorten in Frage kamen, wurden über die Studie vollständig aufgeklärt und um ihre Einwilligung zur Teilnahme gebeten (Patientenaufklärung: Anhang A.6, S.82f.). Die Anonymität der Patientendaten blieb erhalten, da die Zusammenführung lediglich über eine Patienten-Kennnummer 
erfolgte. Ein positives Votum der Ethikkommission und des Datenschutzbeauftragten lagen vor. 


\section{$5 \quad$ Ergebnisse}

\subsection{Beschreibung der Patientengruppen}

\subsubsection{Patienten der poststationären Gruppe}

Durch eine Vorauswahl anhand der Diagnose „Herzinsuffizienz" und nach Einsicht in die Krankenakten kamen 302 der stationären Patienten für die Untersuchung in Frage. Nach Abschluss der Rekrutierung konnten insgesamt 91 der angesprochenen Patienten für die Studie gewonnen werden. Im Nachhinein mussten zwei Patienten wegen fehlender Dokumentation der EF ausgeschlossen werden. In einem weiteren Falle betrug die EF $69 \%$ und entsprach damit nicht den Einschlusskriterien, sodass zu Beginn der Erhebung letztendlich die Daten von 88 Patienten zur Verfügung standen, die sich poststationär auf 80 Praxen verteilten.

Nach zwölf Monaten, d.h. zum Zeitpunkt der zweiten Datenaufnahme, hatte sich die Patientenzahl in der poststationären Kohorte auf 73 Teilnehmer reduziert (Tabelle 8). Sieben Patienten waren aufgrund kardiovaskulärer Erkrankungen verstorben, ein Patient war dialysepflichtig und ein weiterer nach Angaben der Angehörigen schwer pflegebedürftig geworden. Des Weiteren sahen sich zwei Patienten nicht in der Lage, die ihnen zugeschickten Fragebögen auszufüllen und vier Patienten wollten im Telefoninterview ohne Angabe von Gründen nicht mehr an der Studie teilnehmen.

Tabelle 8 : Kohorten im Verlauf der Datenerhebung

\begin{tabular}{lcccccc}
\hline \hline \multicolumn{1}{c}{ Zeitpunkt } & \multicolumn{3}{c}{ Poststationäre Kohorte } & \multicolumn{3}{c}{ Kontrollgruppe } \\
& $n$ & $\%$ & $\begin{array}{c}\text { Anzahl der } \\
\text { Praxen }\end{array}$ & $n$ & $\%$ & $\begin{array}{c}\text { Anzahl der } \\
\text { Praxen }\end{array}$ \\
\hline $\begin{array}{l}\text { Zeitpunkt Null } \\
\text { (Baseline-Erhebung) }\end{array}$ & 88 & 100 & 80 & 88 & 100 & 33 \\
$\begin{array}{l}12 \text { Monate } \\
\text { (Follow-Up-Erhebung) }\end{array}$ & 73 & 83 & 67 & 86 & 98 & 32 \\
\hline \hline
\end{tabular}




\subsubsection{Patienten der Kontrollgruppe}

Im Rahmen des MedVip-Teilprojektes (Abschnitt 4.2.2, S.21) wurden insgesamt 1093 Personen in die ursprüngliche Studie eingeschlossenen. 422 gaben ihre Einwilligung zur Fragebogenuntersuchung. 59 Patienten wurden nachträglich ausgeschlossen. 21 von ihnen widerriefen nachträglich die zuvor ausgesprochene Einwilligung, von 34 Patienten wurde der Fragebogen nicht zurückgesandt, bei zwei Patienten erwies sich die Diagnose Herzinsuffizienz als falsch und bei zwei weiteren Patienten konnte eine Befragung aufgrund sprachlicher Barrieren nicht durchgeführt werden.

Für die vorliegende Studie wurden aus diesem Pool von 363 Patienten 88 Patienten als Kontrollgruppe nach den genannten Kriterien passend zur poststationären Gruppe ausgewählt.

In Verlauf reduzierte sich die Zahl von anfänglich ebenfalls 88 Patienten aus 33 Hausarztpraxen auf 86 Patienten, da zwei von ihnen im Verlauf von zwölf Monaten verstorben waren (Tabelle 8, S.30).

\subsection{Vergleich der Patientengruppen bezüglich entscheidender soziodemographischer und klinischer Daten}

Bei der Auswahl der Patienten der Kontrollgruppe wurde großer Wert auf die Parallelisierung der beiden Gruppen bezüglich wesentlicher Parameter gelegt. Die statistische Überprüfung möglicher Gruppenunterschiede ergab für keine der untersuchten Variablen einen signifikanten Unterschied (t-Test oder $\chi^{2}$-Test; alle $p$-Werte $>0,08$; Tabelle 9, S.32).

Die Mehrheit der Patienten waren zwischen 60 und 80 Jahren alt. Teilnehmer der poststationären Kohorte waren im Mittel 71 Jahre alt; in der Kontrollgruppe betrug das Durchschnittsalter 72 Jahre. Es wurden in beiden Gruppen etwa fünfmal soviel Männer wie Frauen untersucht (74:14).

Ein großer Anteil der Patienten litt an leichteren Formen von chronischer Herzinsuffizienz. Knapp 80\% der Patienten in beiden Gruppen waren den NYHA-Stadien I und Il zuzuordnen. Rund ein Fünftel der Teilnehmer in jeder Gruppe waren schwer erkrankt (NHYA III und IV). 
Als häufige kardiovaskuläre Komorbiditäten bestanden bei über der Hälfte der Patienten eine koronare Herzkrankheit (poststationäre Kohorte: 53\%, Kontrollgruppe: $39 \%$ ) und/oder eine arterielle Hypertonie (57\% vs. 67\%). Ein Diabetes mellitus konnte bei rund einem Drittel der Patienten gefunden werden (22\% vs. 30\%); Niereninsuffizienz ( $2 \%$ in beiden Gruppen) und chronisch obstruktive Lungenerkrankungen (5\% vs. $9 \%$ ) als relative Kontraindikationen verschiedener kardiovaskulärer Medikamente spielten eher eine untergeordnete Rolle. Eine detaillierte Ausführung der Daten kann Tabelle 9 entnommen werden.

Tabelle 9 : Soziodemographische und klinische Charakteristika der poststationären und der Kontrollgruppe zur Baseline-Erhebung

\begin{tabular}{|c|c|c|c|c|c|}
\hline Charakteristika & $\begin{array}{c}\text { Post- } \\
\text { stationäre } \\
\text { Patienten } \\
(n=88)\end{array}$ & $\begin{array}{c}\text { Patienten } \\
\text { der Kon- } \\
\text { trollgruppe } \\
\quad(n=88)\end{array}$ & Statis & stik $^{*}$ & P-Wert \\
\hline Alter, Jahre, MW $( \pm S D)$ & $70,5( \pm 10,7)$ & $72,1( \pm 8,4)$ & $\mathrm{t}[174]=$ & -1.42 & 0.157 \\
\hline Geschlecht, m:w & $74: 14$ & $74: 14$ & $\chi^{2}[1]=$ & 0.00 & 1.000 \\
\hline Schulabschluss, HS:RS:Abi ${ }^{1}, \mathrm{n}$ & $61: 13: 11$ & $67: 13: 5$ & $\chi^{2}[2]=$ & 2.53 & 0.282 \\
\hline NYHA-Klasse, $1: 2: 3: 4^{2}, \mathrm{n}$ & $41: 27: 10: 10$ & $42: 27: 15: 4$ & $\chi^{2}[3]=$ & 3.58 & 0.310 \\
\hline KHK, \% & 53 & 39 & $\chi^{2}[1]=$ & 3.04 & 0.081 \\
\hline Hypertonie, \% & 57 & 67 & $\chi^{2}[1]=$ & 1.41 & 0.236 \\
\hline Diabetes mellitus, \% & 22 & 30 & $\chi^{2}[1]=$ & 1.26 & 0.262 \\
\hline Hyperlipidämie, \% & 33 & 32 & $\chi^{2}[1]=$ & 0.03 & 0.863 \\
\hline Kompensierte Niereninsuffizienz, \% & 2 & 2 & $\chi^{2}[1]=$ & 0.05 & 0.830 \\
\hline COPD, $\%$ & 5 & 9 & $\chi^{2}[1]=$ & 1.06 & 0.303 \\
\hline
\end{tabular}

${ }^{*} \mathrm{t}$-Test oder $\chi^{2}$-Test.

KHK: Koronare Herzkrankheit, COPD: Chronische obstruktive Lungenkrankheit, MW: Mittelwert, SD: Standardabweichung, n: Anzahl der Patienten (absolute Werte)

${ }^{1}$ HS: Volksschul-/Hauptschulabschluss, RS: Realschulabschluss, Abi: Hochschulreife.

2 Selbstauskunft des Patienten: 1: keine Luftnot; 2: Luftnot bei leichter körperlicher Belastung; 3: Luftnot bei geringster körperlicher Belastung; 4: Luftnot in Ruhe. 


\section{3 Überblick über die kardiovaskuläre Medikation im zeitlichen Verlauf}

\subsubsection{Verwendete Einzelstoffe der kardiovaskulären Medikation}

Erhoben wurden alle verordneten Medikamente aus den Entlassungsbriefen (poststationäre Kohorte) oder der zum damaligen Zeitpunkt aktuellen hausärztlichen Medikation (Kontrollgruppe) zum Zeitpunkt Null (Baseline-Erhebung) und durch Telefoninterviews nach zwölf Monaten (beide Gruppen, Follow-Up-Erhebung). Zur Auswertung für die vorliegende Arbeit ausgewählt wurden aber nur diejenigen Stoffgruppen, die relevant für die Behandlung der chronischen Herzinsuffizienz sind. Beachtung fanden weiterhin Medikamente mit Einfluss auf das Herzkreislaufsystem, um einerseits mögliche Medikamenteninteraktionen abschätzen zu können und um andererseits eventuell Rückschlüsse aus dem generellen Verschreibungsverhalten von Ärzten ziehen zu können. Von besonderem Interesse waren die Veränderungen der nachweislich prognoseverbessernden Wirkstoffe im Vergleich zur übrigen Medikation innerhalb des Beobachtungszeitraums. Eine Auflistung aller in der vorliegenden Studie identifizierten Einzelsubstanzen ist Tabelle 10 (S.34) zu entnehmen. 
Tabelle 10 : Einzelsubstanzen der Wirkstoffgruppen

\begin{tabular}{|c|c|}
\hline Stoffgruppe & Einzelstoffe \\
\hline \multirow[t]{7}{*}{ ACE-Hemmer } & Benazepril $^{\star}$ \\
\hline & Captopril ${ }^{*}$ \\
\hline & Enalapril* \\
\hline & Fosinopril $^{*}$ \\
\hline & Lisinopril ${ }^{*}$ \\
\hline & Quinapril* \\
\hline & Ramipril $^{*}$ \\
\hline Aldosteron-Antagonisten & Spironolacton* \\
\hline \multirow[t]{5}{*}{ AT1-Blocker (Sartane) } & Candesartan** \\
\hline & Eprosartan** \\
\hline & Irbesartan** \\
\hline & Losartan** \\
\hline & Valsartan ${ }^{* *}$ \\
\hline \multirow[t]{5}{*}{ Beta-Blocker } & Atenolol \\
\hline & Bisoprolol $^{*}$ \\
\hline & Carvedilol* $^{*}$ \\
\hline & Metoprolol* $^{*}$ \\
\hline & Nebivolol \\
\hline \multirow[t]{4}{*}{ Diuretika } & Furosemid \\
\hline & HCT \\
\hline & Torasemid \\
\hline & Xipamid \\
\hline \multirow[t]{2}{*}{ Digitalisglykoside } & Digitoxin \\
\hline & Digoxin \\
\hline \multirow[t]{3}{*}{ Nitrate } & Isosorbitmononitrat \\
\hline & Isosorbittrinitrat \\
\hline & Molsidomin \\
\hline \multirow[t]{4}{*}{ Kalzium-Antagonisten } & Felodipin \\
\hline & Lercanidipin \\
\hline & Nifedipin \\
\hline & Verapamil \\
\hline
\end{tabular}

\footnotetext{
*Evidenz-A-Medikamente mit Letalitätsreduktion

${ }^{* *}$ Evidenz-A-Medikamente mit Letalitätsreduktion (alternativer Einsatz bei ACE-HemmerUnverträglichkeit)
} 


\subsubsection{Baseline-Erhebung: Verschreibungshäufigkeit in beiden Gruppen}

In beiden Gruppen waren Diuretika die am häufigsten verordneten Medikamente, wobei zur Baseline-Erhebung in der poststationären Gruppe deutlich mehr diuretisch wirkende Substanzen verordnet wurden als in der Kontrollgruppe (poststationäre Kohorte: $89,8 \%$ vs. Kontrollgruppe: $\left.68,2 \% ; \chi^{2}=6,129, p<0,001\right)$. Schleifendiuretika kamen in beiden Gruppen bei etwa der Hälfte der Patienten zum Einsatz (poststationäre Kohorte: $50,0 \%$ vs. Kontrollgruppe: 47,7\%), gefolgt von Thiaziden in mehr als einem Drittel der Fälle (poststationäre Kohorte: 46,0\% vs. Kontrollgruppe: 28,2\%). Spironolacton wird wegen seiner Bedeutung in der evidenzbasierten Therapie der Herzinsuffizienz gesondert aufgeführt (Abschnitt 5.4, S.37).

Beta-Blocker wurden häufig, insbesondere in der poststationären Gruppe, angewendet. Bei knapp 90\% dieser Patienten wurde eine Therapie mit einem Vertreter dieser Stoffgruppe zumindest stationär begonnen und im Entlassungsbrief als Dauertherapie empfohlen. Die Hälfte aller hausärztlich versorgten Patienten der Kontrollgruppe nahmen einen Beta-Blocker ein (poststationäre Kohorte: $87,5 \%$ vs. Kontrollgruppe: $50,0 \%$ ). Ingesamt wurde auch für diese Stoffgruppe ein hochsignifikanter Unterschied zwischen beiden Gruppen zugunsten der poststationären Kohorte festgestellt $\left(\chi^{2}=28,800, p<0,001\right)$.

Eine Behandlung mit ACE-Hemmern wurde bei drei Viertel der Patienten der poststationären Kohorte $(75,0 \%)$ und bei zwei Drittel der Patienten der Kontrollgruppe $(58,0 \%)$ beobachtet. Dadurch ergaben sich zu Beginn der durchgeführten Untersuchung signifikant höhere Verschreibungsraten für Patienten der poststationären Kohorte $\left(\chi^{2}=6,474, p=0,011\right)$.

AT1-Blocker (Sartane), die als Therapiealternative bei ACE-Hemmer-Intoleranz oder Unverträglichkeit gelten, spielten bei den Verschreibungen in beiden Gruppen eher eine marginale Rolle. Nur durchschnittlich einer von zehn Patienten erhielt einen AT1-Blocker (poststationäre Kohorte: 11,4\% vs. Kontrollgruppe: 13,6\%). Signifikante Unterschiede in der Verschreibungshäufigkeit zwischen den Gruppen konnten hier nicht gefunden werden $\left(\chi^{2}=0,208, p=0,867\right)$.

Die zur Behandlung kardiovaskulärer Erkrankungen eingesetzten Digitalispräparate wurden häufiger in der poststationären Gruppe verordnet. Die Hälfte dieser Patienten erhielten bei Entlassung ein Digoxin- oder Digitoxinpräparat $(49,8 \%)$. Hausärztlich 
behandelte Patienten nahmen weitaus weniger Digitalispräparate ein $(19,3 \%)$, $\left(\chi^{2}=8,844, p=0,003\right)$.

Nitrate wurden in beiden Gruppen ähnlich häufig, bei etwa einem Fünftel der Patienten, angewendet (poststationäre Kohorte: 19,3\% vs. Kontrollgruppe: 18,2\%, $\left.\chi^{2}=0,037, p=0,847\right)$.

Kalzium-Antagonisten fanden in der ausschließlich hausärztlich betreuten Kohorte mehr Verwendung und wurden von Klinikärzten nur noch in Einzelfällen verschrieben (poststationäre Kohorte: $20,5 \%$ vs. Kontrollgruppe: $6,8 \%, \chi^{2}=6,947, p=0,008$ ). Tabelle 11 (S.38) zeigt alle oben dargestellten Daten.

Zusammenfassend kann gesagt werden, dass insbesondere in Bezug auf Substanzen mit prognoseverbessernden Eigenschaften in allen Stoffgruppen zu Beginn der Untersuchung eine signifikant häufigere Verordnung in der poststationären Kohorte erfolgte.

\subsubsection{Follow-Up-Erhebung: Verschreibungshäufigkeit in beiden Gruppen}

Diuretika waren auch zum zweiten Erhebungszeitpunkt (Follow-Up-Erhebung) weiterhin in beiden Kohorten die am häufigsten verordneten Medikamente (poststationäre Kohorte: $86,3 \%$ vs. Kontrollgruppe: $62,8 \%$ ). Da sich die Verschreibungen in beiden Gruppen nur geringfügig änderten, kam es weiterhin zu deutlich mehr Verordnungen in der poststationären Kohorte $\left(\chi^{2}=6,244, p=0,002\right)$.

Beta-Blocker wurden in der poststationären Kohorte weiterhin deutlich häufiger verschrieben. Trotz eines Rückgangs der Verschreibungsquote um elf Prozentpunkte auf $76,7 \%$ in der poststationären Kohorte ergab sich bei weitestgehend unveränderten Verschreibungszahlen in der Kontrollgruppe von $52,3 \%$ nach wie vor auch hier ein hochsignifikanter Unterschied zwischen den Gruppen $\left(\chi^{2}=10,134, p=0,001\right)$.

Für die Stoffgruppe der ACE-Hemmer lässt sich zum zweiten Untersuchungszeitpunkt kein signifikanter Unterschied mehr zur Kontrollgruppe feststellen (58,9\% vs. $\left.61,6 \% ; \chi^{2}=0,122, p=0,726\right)$. Verantwortlich dafür ist ein Verschreibungsrückgang in der Entlassungsmedikation der poststationären Gruppe von $75 \%$ auf $57 \%$ in der Follow-Up-Erhebung bei annähernd konstanten Verschreibungsraten in der Kontrollgruppe (Baseline-Erhebung: 58,0\% Follow-Up-Erhebung: 61,6\%). 
AT1-Blocker spielten auch weiterhin bei Verschreibungsraten zwischen 14\% (poststationäre Kohorte) und 13\% (Kontrollgruppe) in beiden Gruppen eine marginale Rolle. Im Vergleich zur Voruntersuchung ergaben sich keine relevanten Veränderungen, sodass in der Follow-Up-Erhebung wiederum keine signifikanten Unterschiede zwischen den Gruppen gefunden werden konnten $\left(\chi^{2}=0,386, p=0,534\right)$.

Digitalispräparate wurden auch in der Follow-Up-Erhebung in der poststationären Gruppe häufiger verschrieben (poststationäre Gruppe: $35,6 \%$ vs. Kontrollgruppe: $20,0 \%$ ). Trotz eines Verschreibungsrückgangs um $14 \%$ blieb ein signifikanter Unterschied zugunsten der poststationären Gruppe bestehen $\left(\chi^{2}=4,835, p=0,028\right)$. Nitrate wurden nach wie vor annähernd gleich häufig in beiden Gruppen verschrieben (poststationäre Gruppe: $13,7 \%$ vs. Kontrollgruppe: $16,3 \% ; \chi^{2}=0,205, p=0,651$ ). Ebenfalls wenig Veränderungen im Vergleich zur Baseline-Erhebung waren bei den KalziumAntagonisten zu verbuchen. Hierbei kam es noch immer zu mehr Verschreibungen in der Kontrollgruppe $(20,9 \%)$ als in der poststationären Gruppe $(9,6 \%)\left(\chi^{2}=3,833\right.$, $\mathrm{p}=0,05)$. Eine Auflistung der erhobenen Daten zeigt Tabelle 11 (S.38).

Zusammenfassend wurden in der Baseline-Erhebung signifikant mehr Diuretika, Beta-Blocker, ACE-Hemmer und Digitalisglycoside in der poststationären Gruppe als in der Kontrollgruppe verschrieben. Mit Ausnahme der ACE-Hemmer ließen sich diese Mehrverschreibungen auch noch in der Follow-Up-Erhebung nachweisen. 
Tabelle 11 : Verordnung von Medikamenten zum Zeitpunkt Null (Baseline-Erhebung) und nach 12 Monaten (Follow-Up-Erhebung) in beiden Gruppen

\begin{tabular}{|c|c|c|c|c|c|c|}
\hline \multirow{3}{*}{$\begin{array}{l}\text { Wirkstoff } \\
\text { Diuretika }^{1}, \%\end{array}$} & \multicolumn{3}{|c|}{ Baseline-Erhebung } & \multicolumn{3}{|c|}{ Follow-Up-Erhebung } \\
\hline & \multirow{2}{*}{$\begin{array}{c}\text { Poststationäre } \\
\begin{array}{c}\text { Patienten } \\
(\mathrm{n}=88)\end{array} \\
89,8\end{array}$} & \multicolumn{2}{|c|}{$\begin{array}{l}\text { Patienten der } \\
\text { Kontrollgruppe } \\
\qquad(n=88)\end{array}$} & \multirow{2}{*}{$\begin{array}{c}\text { Poststationäre } \\
\begin{array}{c}\text { Patienten } \\
(\mathrm{n}=73)\end{array} \\
86,3\end{array}$} & \multicolumn{2}{|c|}{$\begin{array}{l}\text { Patienten der } \\
\text { Kontrollgruppe } \\
\quad(n=85)\end{array}$} \\
\hline & & 68,2 & ** & & 62,8 & * \\
\hline Beta-Blocker ${ }^{2}, \%$ & 87,5 & 50,0 & ** & 76,7 & 52,3 & ** \\
\hline ACE-Hemmer ${ }^{3}, \%$ & 75,0 & 58,0 & * & 58,9 & 61,6 & \\
\hline AT1-Blocker ${ }^{4}, \%$ & 11,4 & 13,6 & & 16,4 & 12,9 & \\
\hline Digitalispräparate, \% & 49,8 & 19,3 & ** & 35,6 & 20,0 & * \\
\hline Nitropräparate, \% & 19,3 & 18,2 & & 13,7 & 16,3 & \\
\hline Ca-Antagonisten ${ }^{5}, \%$ & 6,8 & 20,5 & ** & 9,6 & 20,9 & ** \\
\hline \multicolumn{7}{|c|}{ Alle Angaben in \% der Gruppe } \\
\hline \multicolumn{7}{|c|}{$\chi^{2}$-Test; ${ }^{*} p<0.05 ;{ }^{* *} p<0.01$} \\
\hline \multicolumn{7}{|c|}{${ }^{1}$ Schleifendiuretika, Thiazide und/oder kaliumsparende Diuretika } \\
\hline \multicolumn{7}{|c|}{2 Betaadrenozeptor-Antagonisten (wirkstoffunabhängig) } \\
\hline \multicolumn{7}{|c|}{${ }^{3}$ Angiotensin-Converting-Enzyme-Inihibitoren (wirkstoffunabhängig) } \\
\hline \multicolumn{7}{|c|}{${ }^{4}$ Angiotensin-II-Rezeptor-Antagonisten (wirkstoffunabhängig) } \\
\hline
\end{tabular}

\subsection{Verordnungshäufigkeiten von Medikamenten mit Verbesse- rung der Prognose (Evidenz-A-Medikamente)}

Tabelle 12 (S.39) zeigt die Häufigkeiten der Verschreibungen der prognosebessernden Medikamente zur Baseline- und zur Follow-Up-Erhebung. Bezüglich der BetaBlocker wurden in beiden Gruppen zur Baseline-Erhebung überwiegend Evidenz-AWirkstoffe (Metoprolol, Bisoprolol, Carvedilol) verordnet (poststationäre Gruppe: $94,8 \%$ vs. Kontrollgruppe: 84,4\%). Auch in der Follow-Up-Erhebung entschieden sich Hausärzte in der Mehrzahl der Fälle für diese Substanzen (poststationäre Gruppe: 92,9\% vs. Kontrollgruppe: 86,7\%). Im Gruppenvergleich zeigt sich, dass in der poststationären Kohorte sowohl zum ersten Zeitpunkt als auch zwölf Monate später signifikant mehr dieser Medikamente verordnet wurden als in der Kontrollgruppe (Baseline-Erhebung: poststationäre Gruppe: 83,0\% vs. Kontrollgruppe: 42,0\%; Follow-UpErhebung: poststationäre Gruppe: $71,2 \%$ vs. Kontrollgruppe: 45,3\%). Zu beiden Er- 
hebungszeitpunkten bestanden signifikante Unterschiede zwischen den Gruppen (Baseline-Erhebung: $\quad \chi^{2}=30,817, \quad p<0,001$; Follow-Up-Erhebung: $\chi^{2}=11,200$, $p=0,004)$.

Der Aldosteron-Antagonist Spironolacton wurde zu Beginn der Studie in der poststationären Kohorte sechsmal häufiger verschrieben als in der Kontrollgruppe $\left(\chi^{2}=22,290, p<0,001\right)$. Trotz eines Rückgangs um 13\% fielen die Verordnungen auch hier nach zwölf Monaten nicht auf das Niveau der Kontrollgruppe ab, sodass weiterhin signifikante Unterschiede bestanden $\left(\chi^{2}=8,762, p<0,002\right)$.

Tabelle 12 : Verschreibung von Medikamenten mit Verbesserung der Prognose

\begin{tabular}{|c|c|c|c|c|c|c|}
\hline \multirow[b]{2}{*}{ Wirkstoff } & \multicolumn{3}{|c|}{ Baseline-Erhebung } & \multicolumn{3}{|c|}{ Follow-Up-Erhebung } \\
\hline & $\begin{array}{c}\text { Poststationäre } \\
\text { Patienten } \\
(n=88)\end{array}$ & $\begin{array}{l}\text { Patiente } \\
\text { Kontrollg } \\
\quad(n=8\end{array}$ & & $\begin{array}{c}\text { Poststationäre } \\
\text { Patienten } \\
(n=73)\end{array}$ & $\begin{array}{l}\text { Patienter } \\
\text { Kontrollgr } \\
\quad(n=8\end{array}$ & \\
\hline $\begin{array}{l}\text { Beta-Blocker }^{1} \\
\text { (nur Evidenz A), \% }\end{array}$ & 83,0 & 42,0 & ** & 71,2 & 45,3 & ** \\
\hline Spironolacton, \% & 34,1 & 5,7 & ** & 21,9 & 5,9 & ** \\
\hline ACEI ${ }^{2}$ oder $A T 1^{3}$-Antagonist, $\%$ & 86,4 & 70,5 & ** & 75,3 & 74,4 & \\
\hline \multicolumn{7}{|l|}{ Alle Angaben in \% der Gruppe } \\
\hline \multicolumn{7}{|l|}{$\chi^{2}$-Test; ${ }^{*} \mathrm{p}<0.05 ;{ }^{*} \mathrm{p}<0.01$} \\
\hline \multicolumn{7}{|c|}{${ }^{1}$ Betaadrenozeptor-Antagonisten (nur Metoprolol, Bisoprolol, Carvedilol) } \\
\hline \multicolumn{7}{|c|}{${ }^{2}$ Angiotensin-Converting-Enzyme-Inhibitoren (alle Wirkstoffe) } \\
\hline${ }^{3}$ Angiotensin-II-1-Rezeptor-Ant & agonisten (alle $\mathrm{V}$ & (irkstoffe) & & & & \\
\hline
\end{tabular}

Beschrieben wurde bereits der Verlauf der Behandlung mit ACE-Hemmern (Abschnitt 5.3.3, S.36; Tabelle 11, S.38). In der Follow-Up-Untersuchung kam es zu einem deutlichen Rückgang der Verschreibungszahlen in der poststationären Kohorte, was sich als eine Angleichung an die Verordnungsraten der Kontrollgruppe ausdrückte (poststationäre Gruppe: 58,9\% vs. Kontrollgruppe: 61,6\%). Die in der BaselineUntersuchung signifikanten Unterschiede in der Verschreibungshäufigkeit konnten nach zwölf Monaten nicht mehr festgestellt werden

In diesem Zusammenhang wurde die Stoffgruppe der AT1-Blocker untersucht, da sie im Falle einer ACE-Hemmer-Unverträglichkeit als Ersatzmedikamente anzuwenden sind. Inwiefern die Verordnung von AT1-Blockern als Alternative zur Behandlung mit 
ACE-Hemmern in der vorliegenden Studie Bedeutung erlangte, ist in Tabelle 12 (S.39) dargestellt. Die Verschreibung von Sartanen erfolgte in den meisten Fällen in Abwesenheit eines ACE-Hemmers. Nur in einem Fall wurden Präparate beider Stoffgruppen gemeinsam verordnet. Zu Beginn der Erhebung erhielten auch unter Berücksichtigung der Verschreibungshäufigkeit entweder eines ACE-Hemmers oder alternativ eines AT1-Blockers signifikant mehr Patienten der poststationären Kohorte einen Vertreter der beiden Stoffgruppen $(86,4 \%)$ als Patienten der Kontrollgruppe $(70,5 \%)\left(\chi^{2}=6,578, p=0,01\right)$. Nach Ablauf eines Jahres erhielten noch jeweils drei Viertel der Patienten beider Gruppen Verordnungen aus den genannten Stoffgruppen (poststationäre Kohorte: $75,3 \%$ vs. Kontrollgruppe: $74,4 \%$ ). Damit bestanden trotz der leicht erhöhten Verschreibungsrate der Sartane in der poststationären Gruppe in der Follow-Up-Erhebung um fünf Prozentpunkte weiterhin keine signifikanten Unterschiede in den Verordnungen $\left(\chi^{2}=0,018, p=0,894\right)$. Es konnte also auch unter Berücksichtigung der AT1-Blocker kein signifikanter Unterschied in der Verschreibungshäufigkeit von Substanzen mit Wirkung auf das Renin-Angiotensin-AldosteronSystem zwischen den Gruppen nach einem Jahr gefunden werden.

Nach weiteren möglichen Gründen für einen Therapieabbruch mit ACE-Hemmern wurde in Fällen gesucht, in denen eine ACE-Hemmer-Therapie zwar bei Entlassung bestand, in der Follow-Up-Erhebung aber nicht mehr gefunden werden konnte $(n=15)$. Im Telefoninterview wurden die Patienten standardmäßig im Falle von Absetzen von Medikamenten oder bei Neuverordnungen zu den Gründen für diese Maßnahmen befragt (Abschnitt 4.3, S.22). Die von den Patienten aus einer Liste auszuwählenden bzw. frei zu formulierenden Gründe sind ihrer Häufigkeit nach in Tabelle 13 (S.41) aufgeführt. Es zeigte sich, dass in der Hälfte der Fälle ACE-Hemmer ohne erkennbaren Grund bzw. ohne für den Patienten ersichtliche Gründe durch den Hausarzt absetzt wurden. Von sechs Patienten wurden Gründe wie Unverträglichkeit oder Ersatz durch einen AT1-Blocker (wie oben beschrieben) angegeben. 
Tabelle 13 : Gründe für den Therapieabbruch mit ACE-Inhibitoren in der poststationären Kohorte ( $n=15$ von 43 )

\begin{tabular}{|c|c|c|}
\hline \multicolumn{2}{|c|}{ Grund des Therapieabbruchs nach Selbstauskunft des Patienten } & $n$ \\
\hline „,nicht mehr vertragen“ & & $\overline{11}$ \\
\hline „verschlechterte Nierenfunktion“ & & 1 \\
\hline „ersetzt durch AT1-Blocker“ & & 4 \\
\hline & Gesamt (n) & 6 \\
\hline „durch Hausarzt ersatzlos gestrichen ohne Angabe von Gründen“ & & 6 \\
\hline „abgesetzt, da laut Hausarzt nicht mehr nötig“ & & 2 \\
\hline „Patient hat alle Medikamente selbst abgesetzt“ & & 1 \\
\hline & Gesamt (n) & 9 \\
\hline
\end{tabular}

\subsection{Dosierungsbereiche mit Blick auf Zieldosierungen aus Emp- fehlungen der Leitlinien}

In der vorliegenden Studie wurden neben den erwähnten Parametern auch die Einzeldosierungen ausgewählter Evidenz-A-Medikamente (morgens, mittags, abends, zur Nacht) erfragt. Mithilfe der Einzeldosis der Präparate wurden anschließend die kumulativen Tagesdosen berechnet. Für die Auswertung der Tagesdosen standen nicht von allen Patienten Daten zu Verfügung (Abschnitt 6.3.2, S.61). Die Anzahl der Patienten mit vollständiger Datenerhebung ist in Tabelle 14 (S.43) jeweils für die einzelnen Gruppen in Klammern und kursiver Schrift dargestellt.

Untersucht wurden Tagesdosen von ACE-Hemmern und Beta-Blockern. Die Referenzwerte wurden der Hausärztlichen Leitlinie Chronische Herzinsuffizienz (Leitliniengruppe Hessen 2003) entnommen.

Bei der Behandlung mit ACE-Hemmern wurden die Mehrzahl der Patienten im Untersuchungszeitraum nicht im Zieldosisbereich eingestellt. Mit der Entlassungsmedikation wurden noch die Hälfte der Patienten (51\%) im Bereich der empfohlenen Zieldosen und 39\% mit mittleren Dosen behandelt. In der Follow-Up-Erhebung hatten sich die Verordnungen hauptsächlich in den Mitteldosis-Bereich (54\%) verschoben. Nur noch $37 \%$ der Patienten waren im Bereich der Zieldosierungen eingestellt. Dabei schienen zwischen den Substanzen Unterschiede zu bestehen. Während Enalapril 
mit einer mittleren Tagesdosis von 18 mg/d meist im Zieldosisbereich (20mg/d) verordnet wurde, so kam es bei Lisinopril mit durchschnittlich 11 mg/d (35 mg/d) im Mittel zu einer deutlichen Unterschreitung der empfohlenen Zieldosierung.

Beta-Blocker wurden zu beiden Untersuchungszeitpunkten bei einem Fünftel der Patienten im Zieldosisbereich verordnet. Es kam im Untersuchungszeitraum in der Mehrzahl der Fälle nicht zur Dosissteigerung bis in den Zielbereich. Bei Entlassung erhielten gut die Hälfte der Patienten Beta-Blocker im Mitteldosisbereich (55\%), ein Fünftel $(21 \%)$ wurde hochdosiert behandelt. In der Follow-Up-Erhebung waren die Verschreibungen im Mitteldosisbereich leicht angestiegen (62\%), der Anteil an Patienten mit Hochdosistherapie blieb konstant (20\%). Bei den Beta-Blockern bestanden geringfügige Unterschiede zwischen den einzelnen Substanzen. Bei Metoprolol als meistverwendetem Vertreter zeigte sich eine Verschiebung der Verschreibungen aus dem Niedrig- in den Mitteldosisbereich hinein. Während zum Entlassungszeitpunkt noch gut ein Drittel der Patienten (37\%) mit Minimaldosen und knapp die Hälfte (47\%) mit mittleren Dosen eingestellt war, wurden nach einem Jahr weiniger Patienten mit niedrigen Dosen (jetzt 15\%) und mehr Patienten mit mittleren Dosierungen (jetzt 70\%) behandelt. Der mittlere Wert der verordneten Tagesdosis $(91 \mathrm{mg} / \mathrm{d}$ ) erhöhte sich jedoch in der Follow-Up-Erhebung nur wenig $(105 \mathrm{mg} / \mathrm{d})$ und erreichte nicht den Zieldosisbereich von 200mg/d. Bei den übrigen Substanzen ergaben sich in beiden Untersuchungen gleichbleibend in etwa Tagesdosen im Bereich der halben Zieldosis. Alle Werte sind in Tabelle 14 (S.43) dargestellt.

Zusammenfassend kann festgehalten werden, dass ACE-Hemmer in der poststationären Gruppe bei Entlassung in der Hälfte der Fälle hochdosiert verordnet wurden. Eine weitere Dosissteigerung im Untersuchungszeitraum fand eher nicht statt.

Bei der Verordnung von Beta-Blockern ergab sich im Durchschnitt ebenfalls keine bedeutsame Veränderung in den Dosierungen im Untersuchungszeitraum. 20\% der Patienten wurden hochdosiert, zwei Drittel der Patienten beider Gruppen wurden mit mittleren Dosen behandelt. 
Tabelle 14 : Dosierung von ACE-Inhibitoren und Betaadrenozeptor-Antagonisten in Bezug auf empfohlene Zieldosierungen

\begin{tabular}{|c|c|c|c|c|c|}
\hline \multirow[b]{2}{*}{ Wirkstoff } & \multirow[b]{2}{*}{ Zieldosis $^{3}$} & \multicolumn{2}{|c|}{ Baseline-Erhebung } & \multicolumn{2}{|c|}{ Follow-Up-Erhebung } \\
\hline & & $\begin{array}{c}\text { Poststationäre } \\
\text { Patienten }\end{array}$ & $\begin{array}{l}\text { Patienten der } \\
\text { Kontrollgruppe }\end{array}$ & $\begin{array}{c}\text { Poststationäre } \\
\text { Patienten }\end{array}$ & $\begin{array}{l}\text { Patienten der } \\
\text { Kontrollgruppe }\end{array}$ \\
\hline ACEH,gesamt ${ }^{1}$ & & $51: 39: 10(n=59)$ & $46: 39: 15(n=39)$ & $37: 54: 9(n=35)$ & $53: 29: 18(n=38)$ \\
\hline Enalapril $^{1}$ & & $69: 31: 0(n=13)$ & $62: 23: 15(n=13)$ & $75: 25: 0(n=8)$ & $64: 14: 22(n=14)$ \\
\hline $\mathrm{MW} \pm \mathrm{SD}, \mathrm{mg} / \mathrm{d}^{2}$ & 20 & $17,7 \pm 9,7$ & $16,2 \pm 10,4$ & $16,9 \pm 5,9$ & $16,3 \pm 10,3$ \\
\hline Ramipril & & $53: 40: 7(n=30)$ & $56: 33: 11(n=9)$ & $38: 54: 8(n=13)$ & $60: 30: 10(n=10)$ \\
\hline $\mathrm{MW} \pm \mathrm{SD}, \mathrm{mg} / \mathrm{d}^{2}$ & 10 & $6,8 \pm 3,3$ & $6,0 \pm 3,3$ & $8,4 \pm 7,1$ & $7,6 \pm 5,5$ \\
\hline Benazepril & & $100: 0: 0(n=2)$ & $75: 25: 0(n=4)$ & $50: 50: 0(n=2)$ & $75: 25: 0(n=4)$ \\
\hline $\mathrm{MW} \pm \mathrm{SD}, \mathrm{mg} / \mathrm{d}^{2}$ & 10 & $10,0 \pm 0$ & $26,3 \pm 17,0$ & $7,5 \pm 3,5$ & $20,6 \pm 15,3$ \\
\hline Lisinopril $^{1}$ & & $21: 50: 29(n=14)$ & $10: 70: 20(n=10)$ & $8: 75: 17(n=12)$ & $22: 44: 34(n=9)$ \\
\hline $\mathrm{MW} \pm \mathrm{SD}, \mathrm{mg} / \mathrm{d}^{2}$ & 35 & $11,1 \pm 5,6$ & $15,3 \pm 10,7$ & $14,8 \pm 7,3$ & $15,6 \pm 12,4$ \\
\hline Captopril & & $(n=0)$ & $33: 33: 33(n=3)$ & $(n=0)$ & $0: 100: 0(n=1)$ \\
\hline $\mathrm{MW} \pm \mathrm{SD}, \mathrm{mg} / \mathrm{d}^{2}$ & 150 & - & $81,3 \pm 65,7$ & - & 68,0 \\
\hline $\mathrm{BB}$, gesamt $^{1}$ & & $21: 55: 24(n=63)$ & $22: 65: 13(n=31)$ & $20: 62: 18(n=45)$ & $15: 67: 18(n=33)$ \\
\hline Metoprolol $^{1 *}$ & & $16: 47: 37(n=30)$ & $25: 75: 25(n=16)$ & $15: 70: 15(n=27)$ & $19: 44: 37(n=16)$ \\
\hline $\mathrm{MW} \pm \mathrm{SD}, \mathrm{mg} / \mathrm{d}^{2}$ & 200 & $91,4 \pm 51,5$ & $107,6 \pm 66,8$ & $104,9 \pm 45,3$ & $91,8 \pm 60,0$ \\
\hline Bisoprolol $^{1}$ & & $24: 71: 5(n=21)$ & $0: 100: 0(n=8)$ & $36: 45: 19(n=11)$ & $0: 100: 0(n=10)$ \\
\hline $\mathrm{MW} \pm \mathrm{SD}, \mathrm{mg} / \mathrm{d}^{2}$ & 10 & $5,2 \pm 2,8$ & $4,1 \pm 1,3$ & $5,5 \pm 3,1$ & $4,0 \pm 1,3$ \\
\hline Carvedilol $^{1}$ & & $25: 50: 25(n=12)$ & $43: 57: 0(n=7)$ & $14: 57: 29(n=7)$ & $29: 71: 0(n=7)$ \\
\hline $\mathrm{MW} \pm \mathrm{SD}, \mathrm{mg} / \mathrm{d}^{2}$ & 50 & $22,5 \pm 16,1$ & $26,8 \pm 15,2$ & $22,0 \pm 14,7$ & $26,1 \pm 13,6$ \\
\hline
\end{tabular}

ACEH: ACE-Hemmer, BB: Beta-Blocker

1 : $n \geq 75 \%$ der Zieldosis $2: n \geq 25 \%<75 \%$ der Zieldosis $3: n<25 \%$ der Zieldosis, alle Angaben in $\%$ der Gruppe

${ }^{2}$ Mittelwert und Standardabweichung der kumulativen Tagesdosierungen

${ }^{3}$ Zielbereich kumulativer Tagesdosierungen nach Empfehlungen der Leitliniengruppe Hessen (2003) in $\mathrm{mg} / \mathrm{d}$

\subsection{Hospitalisierung und Krankheitsverlauf im Untersuchungs- zeitraum}

Krankenhausaufenthalte der Patienten waren von besonderer Bedeutung, da sich hierbei die Möglichkeit einer Therapieoptimierung bietet. 
In der Follow-Up-Untersuchung wurden rückblickend Krankenhausaufenthalte aufgrund der bestehenden Herzerkrankung, deren Häufigkeit, die Aufenthaltsdauer sowie Gründe für den Aufenthalt bzw. die durchgeführten Maßnahmen in beiden Kohorten erfasst (Tabelle 15, S.45). Es wurden mehr Patienten der poststationären Kohorte stationär behandelt als Patienten der Kontrollgruppe ( $40 \%$ vs. $7 \%$ ). Darüber hinaus wurden Patienten der poststationären Kohorte im Untersuchungszeitraum insgesamt anderthalb mal so häufig stationär behandelt wie Patienten der Kontrollgruppe $(1,6: 1)$. Die durchschnittliche Liegezeit war in der poststationären Kohorte etwa doppelt so hoch (14 Tage vs. 8 Tage).

Grund der Aufnahme waren in beiden Kohorten vornehmlich eine Umstellung der Therapie bzw. eine Akutmedikation bei Zeichen einer zunehmenden Dekompensation ( $n=8$ vs. $n=4)$. In der poststationären Kohorte waren darüber hinaus Herzkatheteruntersuchungen mit anschließender Perkutaner Transluminaler Coronarer Angioplastie (PTCA) $(n=8)$, die Versorgung mit einem implantierbaren CardioverterDefibrillator (ICD) $(n=6)$ sowie in einem Fall die Implantation eines Herzschrittmachers vonnöten. In den übrigen Fällen handelte es sich um kardiologische Interventionen im weitesten Sinne wie Kontrollen des ICD wegen Fehlfunktion oder Batteriewechsel des Herzschrittmachers.

Patienten der poststationären Kohorte befanden sich also zahlreicher, häufiger und länger in stationärer Behandlung als Patienten der Kontrollgruppe. 
Tabelle 15 : Krankenhausaufenthalte der Patienten im Untersuchungszeitraum von zwölf Monaten

\begin{tabular}{lcc}
\hline \hline & $\begin{array}{c}\text { Poststationäre Kohorte } \\
\text { (Follow-Up-Erhebung: } n=73)\end{array}$ & $\begin{array}{c}\text { Kontrollgruppe } \\
\text { (Follow-Up-Erhebung: } n=86)\end{array}$ \\
\hline Hospitalisierungsrate $^{1}, \mathrm{n}(\%)$ & $29(39,7)$ & $6(7,0)$ \\
Klinikaufenthalte $^{2}, \mathrm{n}$ & $1,6( \pm 1,1)$ & $1( \pm 0)$ \\
Liegezeit $^{3}$, Tage & $13,7 \mathrm{~d}( \pm 12,5 \mathrm{~d})$ & $7,7( \pm 6,9)$ \\
Grund des KH-Aufenthaltes, $\mathrm{n}(\%)$ & & $4(66,7)$ \\
$\quad$ nur Medikamente & $8(27,6)$ & $1(16,7)$ \\
Herzkatheter/PTCA & $8(27,6)$ & $1(16,7)$ \\
SM-Implantation & $1(3,4)$ & 0 \\
ICD-Implantation & $6(20,7)$ & 0 \\
Andere & $6(20,7)$ & \\
\hline \hline
\end{tabular}

Angaben in Mittelwert ( \pm Standardabweichung), wenn nicht anders angegeben

PTCA: Perkutane Transluminale Coronare Angioplastie, SM: Herzschrittmacher, ICD: implantierbarer Cardioverter-Defibrillator

${ }^{1}$ Anteil der Patienten mit Klinikaufenthalt im Untersuchungszeitraum von einem Jahr

${ }^{2}$ durchschnittliche Anzahl der Klinikaufenthalte aller im Untersuchungszeitraum hospitalisierten Patienten

${ }^{3}$ durchschnittliche Anzahl der Krankenhaustage während aller Krankenhausaufenthalte im Untersuchungszeitraum in Tagen

${ }^{4}$ Kontrolle des ICD, Batteriewechsel des SM 


\section{Diskussion}

\subsection{Zielsetzung und Hauptergebnisse der Studie}

In der vorliegenden Arbeit wurden zwei Gruppen von Patienten mit Herzinsuffizienz bezüglich der Qualität der medikamentösen Therapie miteinander verglichen. Die poststationäre Gruppe wurde einmal zum Zeitpunkt ihrer Entlassung aus der Klinik und nach zwölf Monaten hausärztlicher Versorgung untersucht. Patienten der Kontrollgruppe befanden sich zu beiden Zeitpunkten in hausärztlicher Behandlung. Ziel der Studie war es, zu überprüfen, ob durch eine Sicherung der Diagnose und die Empfehlung einer Therapie in der Klinik die Qualität der hausärztlichen Pharmakotherapie bei Patienten mit Herzinsuffizienz dauerhaft verbessert werden kann.

Wir konnten nachweisen, dass sowohl mehr prognoseverbessernde Medikamente (z.B. Beta-Blocker), als auch mehr symptomatische Wirkstoffe (z.B. Diuretika) verordnet wurden. Die Verordnungen überdauerten zudem den Untersuchungszeitraum. Die höheren Verschreibungsraten, die mit einer mehr leitliniengerechten Behandlung verknüpft sind, waren statistisch signifikant höher in der poststationären Kohorte als die Vergleichswerte in der Kontrollgruppe. Somit schienen Hausärzte und Patienten in der Tat von der Beseitigung der diagnostischen und therapeutischen Unsicherheiten zu profitierten. Allein die Verschreibungsraten der ACE-Hemmer blieben von dem beschriebenem Trend ausgenommen. Während zum Zeitpunkt der Entlassung noch signifikant mehr ACE-Hemmer in der poststationären Kohorte verschrieben wurden, konnten nach Ablauf von zwölf Monaten nur noch Verschreibungsraten auf dem Niveau der Kontrollgruppe gefunden werden. Ein Vergleich beider Gruppen ergab nun keine signifikanten Unterschiede mehr. Des Weiteren konnte gezeigt werden, dass nach den Empfehlungen der Leitlinien für chronische Herzinsuffizienz in beiden Gruppen keine ausreichend hohen Dosen der prognoseverbessernden Substanzen verordnet wurden. Es fand im Mittel keine bedeutsame Dosissteigerung durch den Hausarzt nach der Entlassung aus dem Krankenhaus statt. 


\subsection{Leitlinienadhärenz der Pharmakotherapie an der stationär - ambulanten Schnittstelle}

\subsubsection{Verordnungsraten kardiovaskulärer Medikation im zeitlichen Verlauf}

In der vorliegenden Studie wurden die poststationären Patienten bei Entlassung aus der Klinik zu 83\% mit Beta-Blockern und 75\% mit ACE-Hemmern behandelt. Dagegen wurden in der hausärztlich betreuten Kontrollgruppe bei 50\% der Patienten BetaBlocker und bei 58\% ACE-Hemmer verordnet (Abschnitt 5.3, S.32). Im Rahmen leitliniengerechter Versorgung schien den poststationären Patienten ein Behandlungsvorteil entstanden zu sein.

Betrachtet man zunächst die Empfehlungen aus den Entlassungsbriefen (poststationäre Kohorte, Baseline-Erhebung), so entsprechen diese dem Behandlungsregime im Krankenhaus, wie es von Komajda et al. (2003) untersucht wurde. In dieser Studie wurde die stationäre leitliniengerechte Behandlung von Patienten mit Herzinsuffizienz in 115 Kliniken aus 24 europäischen Ländern an einer Kohorte von insgesamt 11016 Patienten auf der Basis der Entlassungsbriefe erhoben. Bei 2248 von 11016 Patienten wurde eine EF < 40\% festgestellt. In dieser Gruppe erhielten 79,9\% der Patienten einen ACE-Hemmer und 48,9\% der Patienten einen Beta-Blocker. Die Häufigkeiten der Verschreibungen in den von uns untersuchten Entlassungsmedikationen in der poststationären Kohorte stimmen bei den ACE-Hemmern mit den oben erwähnten in etwa überein. Im Falle der Beta-Blocker zeigen sich große Differenzen zugunsten der von uns untersuchten Patienten, die bei Entlassung insgesamt häufiger von Evidenz-A-Beta-Blockern profitieren konnten (poststationäre Gruppe, Baseline-Erhebung: 83,0\% vs. Komajda et al. (2003): 48,9\%).

Die entscheidendere Frage bezieht sich aber auf die hausärztliche Medikation, in der vorliegenden Studie repräsentiert einerseits durch die Kontrollgruppe, andererseits durch die poststationäre Gruppe in der Follow-Up-Untersuchung. Die Verschreibungshäufigkeiten von ACE-Hemmern und Beta-Blockern im primärärztlichen Bereich untersuchten Cleland et al. (Cleland et al. 2002). Das Patientenkollektiv umfasste 11062 Patienten von 1363 Hausärzten aus 15 europäischen Ländern. Es zeigte sich, dass 6637 Patienten (60\%) mit ACE-Hemmern und 3761 Patienten (34\%) mit Beta-Blockern versorgt wurden, was unsere Zahlen in etwa widerspiegeln (Anhang 
A.1, S.78). Höhere Verordnungsraten von prognoseverbessernden Medikamenten in der Klinik und signifikant geringere Verordnungsraten in der hausärztlichen Versorgung, wie in anderen Studien zur Versorgungsforschung beschrieben, konnten auch durch unsere Daten bestätigt werden.

Ein wichtiger Aspekt der vorliegenden Untersuchung ist die Frage nach Veränderungen der Verschreibungen an der stationär-ambulanten Schnittstelle und damit zwischen Entlassungsmedikation und hausärztlichen Verordnungen nach einem Jahr. In der Kontrollgruppe ergaben sich im Verlauf eines Jahres erwartungsgemäß keine signifikanten Veränderungen in den Verordnungen. Dies scheint nicht verwunderlich, da im Beobachtungszeitraum die Versorgung der Patienten beinahe ausschließlich durch den Hausarzt erfolgte.

Anders stellt sich der Sachverhalt in der poststationären Kohorte dar (Abschnitt 5.3, S.33). Nach zwölf Monaten war insgesamt ein milder Rückgang aller Verschreibungen zu beobachten. Lediglich die Verordnung von Sartanen (Baseline-Erhebung: $11,4 \%$ vs. Follow-Up-Erhebung: 16,4\%) sowie von Ca-Antagonisten (6,8\% vs. 9,6\%) nahm nach der stationären Entlassung im Verlauf leicht zu.

Obwohl die Verordnungen insgesamt zurückgingen, ließen sich aber dennoch für alle entscheidenden Medikamente auch in der Follow-Up-Erhebung weiterhin signifikante Unterschiede im Vergleich zur Kontrollgruppe (hier blieben die Verordnungsraten ja weitestgehend konstant) feststellen. Ganz besondere Bedeutung erhält dieser Umstand bei der Betrachtung der prognosebessernden Medikamente, kritisieren doch Autoren vieler Arbeiten die generell mangelhafte Versorgung mit gerade diesen entscheidenden Stoffklassen. Exemplarisch für die Fülle der Untersuchungen sei hier eine Erhebung von Boyles et al. (2004) an 450 neu stationär aufgenommenen Patienten mit Herzinsuffizienz erwähnt. Die Autoren konnten zeigen, dass unter den bei Klinikaufnahme erhobenen Medikamenten nur 50\% der Patienten mit ACEHemmern, 22\% mit Beta-Blockern und 15\% mit Spironolacton behandelt wurden. Auch für symptomatische Medikamente wurden nur unzureichende Verschreibungsraten beschrieben (Schleifendiuretika 65\%, Digoxin 24\%). Nur bei der Hälfte aller mit einem ACE-Hemmer behandelten Patienten zeigten sich Verordnungen im Zieldosisbereich. In der vorliegenden Untersuchung konnten für die Patienten der poststationären Kohorte nicht nur höhere Verschreibungsraten für prognoseverbessernde Medikamente wie Evidenz-A-Beta-Blocker, sondern auch für symptomatische Therapeutika wie Diuretika gefunden werden (Tabelle 11, S.38). Ob den Patienten der 
poststationären Kohorte im Vergleich zur Kontrollgruppe ein wesentlicher Behandlungsvorteil entstand, kann die vorliegende Studie nicht klären. Deutlich war jedoch zumindest der Benefit bezogen auf die Verschreibungshäufigkeit leitliniengerechter Medikation. Die Verschreibungsraten lagen nicht nur im Vergleich zur eigenen Kontrollgruppe, sondern auch im Vergleich zu den bei Cleland et al. (2002) gefundenen Daten wesentlich höher. Allein im Falle der ACE-Hemmer lässt sich zum zweiten Untersuchungszeitpunkt kein signifikanter Unterschied mehr zur Kontrollgruppe feststellen. Die initial noch höhere Verschreibungsquote in der poststationären Gruppe zu Beginn der Studie konnte in der Follow-Up-Untersuchung nicht mehr nachgewiesen werden. Die Rate hatte sich auf das Behandlungsniveau der ausschließlich hausärztlich betreuten Patienten abgesenkt (Tabelle 11, S.38).

Den AT1-Blockern kommt insofern eine besondere Bedeutung zu, da sie im Falle einer ACE-Inhibitor-Unverträglichkeit als Ersatzmedikamente anzuwenden sind. Pitt et al. (2000) konnten in einer vergleichenden Letalitätsstudie zwischen Losartan und Captopril bei Patienten mit systolischer Herzinsuffizienz im Stadium NYHA II-IV keinen Unterschied hinsichtlich der Gesamtsterblichkeit, der plötzlichen Herztodesrate oder der Reanimationshäufigkeit zeigen. Die Studienergebnisse sprachen also für eine Letalitätssenkung unter AT1-Blockern vergleichbar mit der der ACE-Hemmer bei der Behandlung der chronischen Herzinsuffizienz. Lediglich Therapieabbrüche waren unter AT1-Blockern aufgrund einer niedrigeren Nebenwirkungsrate signifikant seltener als unter ACE-Hemmern. Insgesamt konnte aber keine Überlegenheit der AT1Blocker gegenüber den ACE-Hemmern gezeigt werden. Aus diesem Grund sowie aufgrund der guten Langzeiterfahrungen mit der Behandlung durch ACE-Hemmer werden sie weiterhin als primäre Pharmakotherapie der Herzinsuffizienz empfohlen. Sartane sollen nur bei Unverträglichkeitsreaktionen durch ACE-Hemmer zum Einsatz kommen. In welchen Größenordnungen diese Reaktionen (z.B. trockener Reizhusten, angioneurotisches Ödem) auftreten beschrieben Butler et al. (2004) in einer Studie zur Verschreibung von ACE-Hemmern bei 1041 Patienten mit Herzinsuffizienz. Dabei trat bei 81 von 1041 Patienten (8\%), die einen ACE-Hemmer eingenommen hatten eine Allergie oder Unverträglichkeit gegen ACE-Hemmer auf. In der vorliegenden Studie lagen die Verschreibungsraten von AT1-Blockern in etwa in der Größenordnung der zuvor erwähnten Unverträglichkeitszahlen. Jedoch reichten die Verordnungen nicht aus, um die Unterversorgung mit ACE-Hemmern zu kompensieren. Auch unter Berücksichtigung der Verschreibungshäufigkeit entweder eines ACEHemmers oder alternativ eines AT1-Blockers zeigte sich ein deutlicher Rückgang der 
Verschreibungen in der Follow-Up-Erhebung in der poststationären Kohorte bis auf das Niveau der Kontrollgruppe (poststationäre Gruppe: von $86 \%$ auf $75 \%$; Kontrollgruppe Follow-Up-Erhebung: 74\%).

Zusammenfassend gilt festzuhalten, dass in der Stoffgruppe, deren Nutzen als gesichert gilt und deren frühzeitige Anwendung zur Behandlung auch der asymptomatischen Herzinsuffizienz (NYHA I) von Leitlinienautoren gefordert wird (Leitliniengruppe Hessen, S.8), keine dauerhafte Erhöhung der Verschreibungsrate gezeigt werden konnte. Die poststationäre Gruppe, die bei Entlassung ACE-Hemmer oder alternativ AT1-Blocker in höherem Maße erhielt, hatte sich nach zwölfmonatiger hausärztlicher Betreuung der Kontrollgruppe in den Verschreibungsraten angeglichen. Empfehlungen des Klinikpersonals wurden in diesem Falle offenbar nicht ausreichend umgesetzt. Es konnte gezeigt werden, dass in mehr als der Hälfte der Fälle, in denen die Substanzen in der Follow-Up-Erhebung abgesetzt wurden, dieses ohne nachvollziehbare Gründe erfolgte (Abschnitt 5.4, S.38).

\subsubsection{Mögliche Gründe der mangelnden Leitlinienadhärenz}

Bei der vorliegenden Studie wurde das Hauptaugenmerk auf den Verlauf der medikamentösen Behandlung von herzinsuffizienten, poststationären Patienten gelegt. Faktoren, die das hausärztliche Verschreibungsverhalten beeinflussen könnten, wurden nicht in der vorliegenden, wohl aber in anderen Studien untersucht. So fasst McMurray (1998) die Ergebnisse einer Untersuchung zur zurückhaltenden Verordnung von ACE-Hemmern sowie Gründe der Hausärzte wie folgt zusammen:

1. Chronische Herzinsuffizienz wird als bedeutendes Gesundheitsproblem, das als solches eine konsequente Behandlung verdient hätte, unterschätzt.

2. Die deutlichen klinischen Therapieerfolge werden unterschätzt.

3. Das äußerst günstige Kosten-Nutzen-Verhältnis wird unterschätzt.

4. Es wird befürchtet, dass Raten unerwünschter Wirkungen die klinischen Vorteile übersteigen könnten.

5. Es hält sich der Glaube, dass sich die in klinischen Studien gefundenen Vorteile nicht in die Praxis übertragen lassen. 
Im Folgenden sollen einzelne Punkte als Ursachen für die Untersuchungsergebnisse der vorliegenden Arbeit in Bezug auf die Empfehlungen der Leitlinien zur Behandlung von Herzinsuffizienz aufgegriffen und diskutiert werden.

So kann ein möglicher Grund für die niedrigen Verschreibungsraten von ACEHemmern oder/und für den starken isolierten Rückgang der Verordnungen in den Follow-Up-Untersuchungen in spezifischen Begleiterkrankungen der Patienten gesehen werden (Tabelle 9, S.32). Wenngleich auch einige Erkrankungen, wie beispielsweise die arterielle Hypertonie, durchaus Indikationen für ACE-Hemmer darstellen, könnte das Vorliegen von gerade in diesem Kollektiv häufigen oder sogar ursächlichen Leiden, wie z.B. koronare Herzkrankheit (KHK), Diabetes mellitus oder eine Niereninsuffizienz den Hausarzt eventuell vor therapeutische Probleme stellen.

Die Fachinformation (Bundesverband der Pharmazeutischen Industrie 2006) weist darauf hin, dass bei Patienten mit Niereninsuffizienz ACE-Hemmer mit Vorsicht und unter engmaschiger Laborkontrolle anzuwenden seien, da es zu Veränderungen der Nierenfunktion kommen könne. Zudem träten die anderenorts beschriebenen Neutropenie und Agranulozytose unter ACE-Hemmer-Therapie bei niereninsuffizienten $\mathrm{Pa}$ tienten häufiger auf. Auch bei ischämisch bedingter Herzerkrankung (KHK) oder zerebrovaskulärer Verschlusskrankheit könne es aufgrund von starker Hypotonie zu Myokardinfarkt oder Apoplex kommen. Des Weiteren wird auf die Wechselwirkung mit gerade in der Behandlung der Hypertonie oder gar der Herzinsuffizienz selbst häufig verwendeten und bewährten Medikamenten hingewiesen. So sei z.B. Vorsicht geboten bei der gleichzeitigen Einnahme von Diuretika bzw. von anderen Antihypertensiva. Hier könne es zu erhöhten Kaliumspiegeln und anderen Elektrolytentgleisungen sowie zu ausgeprägter Hypotonie mit Folgen für das kardiovaskuläre System kommen. Im Gespräch mit Hausärzten wurde zudem mehrfach die nicht zu unterschätzende Problematik des trockenen Reizhustens genannt. Diese unerwünschte Arzneimittelwirkung der ACE-Hemmer sei häufig (10-15\% laut KV Hamburg) und unangenehm und ein dringlicher Grund für den Patienten das Medikament nicht länger einzunehmen. Es erscheint nur allzu verständlich, dass dem kardiologisch nicht spezialisierten Allgemeinmediziner hier Zweifel an der richtigen Behandlung seiner Patienten insbesondere mit ACE-Hemmern im Gesamtkonzept aufkommen mögen.

Dass gerade die Furcht vor Nebenwirkungen zu einer Zurückhaltung bei der Verordnung der erwähnten Medikamente führen kann, bestätigen auch Hobbs et al. (2000) in einer europaweiten Studie zum Verschreibungsverhalten von Hausärzten. Obwohl 
82\% der befragten Ärzte von der Symptombesserung und sogar $91 \%$ von der Letalitätsreduktion durch ACE-Hemmer überzeugt waren, befürchteten immerhin $51 \%$ mögliche Risiken und 62\% die Gefahr erheblicher unerwünschter Arzneimittelwirkungen durch diese Stoffgruppe.

Ein weiterer Grund für die medikamentöse Unterversorgung hausärztlich betreuter Patienten könnte die fehlende Krankheitseinsicht und mangelnde Compliance der Patienten selbst sein. Diese wiederum kann einerseits in dem Krankheits- und Therapieverständnis des Patienten, aber andererseits auch in einem ungenügend ausgebildeten Arzt-Patienten-Verhältnis begründet liegen (Buddeberg und Willi 1998, S.437f.). Ohne eine genügende Aufklärung des Patienten über seine Erkrankung und den eingeschlagenen therapeutischen Weg kann es schnell zu einer Ablehnung der Therapie beim Auftreten von geringfügigen Nebenwirkungen kommen. Im schlimmsten Fall kommt es überhaupt nicht zur Auseinandersetzung zwischen Arzt und Patient und der Patient setzt die Medikamente selbst ab. Eine Untersuchung von Barat et al. (2001) zur generellen Einnahme von Medikamenten bei 348 hausärztlich betreuten Patienten zeigte eine "Non-Compliance“ zwischen 20 und 70\%, je nach Messmethode. Das Wissen über die Behandlung sowie die Wirkungen und Nebenwirkungen der verordneten Medikamente, über die weniger als $6 \%$ der untersuchten Patienten aufgeklärt waren, spielten hierbei eine große Rolle. Patienten mit Herzinsuffizienz und die Einnahmetreue bezüglich der verordneten Medikation untersuchten Cline et al. (1999). Die Autoren konnten zeigen, dass $27 \%$ der Patienten die verschriebenen Substanzen nicht einnahmen. Auch hierbei wurden Gründe wie Unkenntnis bezüglich der einzunehmenden Dosis (50\%) oder des Zeitpunktes der Einnahme (64\%) angegeben.

Auch für die Therapie mit (in der vorliegenden Arbeit weitaus häufiger verordneten, aber dennoch in der Follow-Up-Erhebung rückläufigen) Beta-Blockern ergeben sich ähnliche Überlegungen wie für ACE-Hemmer. Hier wird in der Fachinformation (Bundesverband der Pharmazeutischen Industrie 2006) z.B. auf die Problematik in Bezug auf ein geschädigtes bzw. hyperreagibles Bronchialsystem (z.B. COPD) hingewiesen. Eine besonders sorgfältige ärztliche Überwachung sei bei Patienten mit Diabetes mellitus angeraten, da es zu schweren hypoglykämischen Zuständen kommen könne. Die Liste der möglichen Wechselwirkungen mit anderen wichtigen Medikamenten zur Behandlung von kardiovaskulären Erkrankungen ist mindestens ebenso lang. 
Interessanterweise wurden entgegen allen Erwartungen nicht nur die Mehrzahl der untersuchten Patienten der poststationären Kohorte mit einem Evidenz-A-BetaBlocker in der Medikationen entlassen; die Therapie mit Vertretern dieser Stoffgruppe wurde auch in wenig verminderter Zahl fortgesetzt (Tabelle 11, S.38). Gerade im Vergleich zu den als besser verträglich geltenden ACE-Hemmern erscheint dies verwunderlich, konnte doch für Beta-Blocker ein breites Spektrum an potentiell gefährlichen Verläufen beschrieben werden. Ein möglicher Grund könnte in der Krankengeschichte der Patienten verborgen liegen. Als Einweisungsgrund finden sich bei einem großen Teil der untersuchten Patienten pectanginöse Beschwerden oder gar ein behandlungsbedürftiger Myokardinfarkt; gefolgt von einer ACVB-Operation oder einer PTCA mit Stent-Implantation. Auch Herzrhythmusstörungen sind bei einem großen Teil der Patienten zu finden. In der Postinfarktprophylaxe und erst recht in der Behandlung einfacher Herzrhythmusstörungen sind Beta-Blocker seit langem fester Bestandteil der Basistherapie (Alexander et al. 1999, 219ff.). Anders als bei der Therapie der chronischen Herzinsuffizienz könnte für diese Indikationen wohl Einigkeit unter den Ärzten über die vorteilhaften Wirkungen der Substanzen bestehen. Man könnte also mutmaßen, dass die hohe Verschreibungsrate dieser Medikamente vielleicht auch unter der Vorstellung der Behandlung von KHK und Rhythmusstörungen durch Hausärzte zustande kommt.

Dennoch wird außerdem von mehreren Autoren generell die ausgesprochen gute Verträglichkeit von z.B. Carvedilol im klinischen Alltag auch beim älteren und multimorbiden Patienten beschrieben (Krum 2004) und der deswegen breite Einsatz der Präparate postuliert.

Als entscheidender Aspekt bei der Behandlung von Patienten mit Herzinsuffizienz wurden im Gespräch mit Hausärzten auch die Budgetierung und die damit im Zusammenhang stehenden Arzneimittelkosten angesehen. Zum Vergleich der Kosten für die medikamentöse Behandlung eines Patienten im Zieldosisbereich wurde eine Gegenüberstellung von häufig verordneten Präparaten aus der Gruppe der ACEHemmer bzw. der Beta-Blocker durchgeführt (Anhang A.3, S.79). Es stellte sich heraus, dass die Verschreibung einer N3-Packung (100 Tabletten) eines Beta-Blockers in der Stärke der jeweiligen Tageszieldosis des entsprechenden Präparates im Durchschnitt mit um etwa 30\% geringeren Kosten verbunden war als die Verordnung einer äquivalenten Menge eines ACE-Hemmers. So kosten beispielsweise 100 Tabletten Enalapril (20 mg) (ein in der vorliegenden Studie häufig verschriebener Wirk- 
stoff) als insgesamt preisgünstigster Vertreter im besten Falle 29,74 Euro. Der am häufigsten verschriebene Beta-Blocker Metoprolol (200 mg/ 100 Tabletten) hingegen verursacht lediglich Kosten in Höhe von 19,64 Euro (ebenfalls günstigstes Präparat). Auch andere ACE-Hemmer wie Ramipril (100 Tabletten/ 10 mg, 44,20 Euro) oder Benazepril (100 Tabletten/ 10 mg, 32,90 Euro) bleiben von diesem Trend nicht ausgenommen. Im Regelfall ist also eine Behandlung mit Beta-Blockern preiswerter als mit ACE-Inhibitoren. So könnten behandelnde Ärzte neben vielen weiteren möglichen Gründen zuletzt auch aufgrund von Kostenkalkulationen und Budgetierung im Zweifelsfalle eher geneigt sein, ACE-Hemmer nicht oder nicht weiter zu verordnen, obwohl bei der Behandlung der Herzinsuffizienz absolut klare Indikationen für die einzelnen Wirkstoffe bestehen.

Eine ganz andere Problematik auf der Suche nach Gründen für die unzureichende Behandlung ergibt sich bereits sehr viel früher - an der stationär-ambulanten Schnittstelle. Es ist durchaus bekannt, dass dieses empfindliche Kontinuum zahlreichen Störungen unterworfen ist. Da ein wesentlicher Bezug der vorliegenden Arbeit auf den Entlassungsbriefen der Klinik lag, ist davon ausgegangen worden, dass diese den Hausarzt auch (zeitgerecht) erreichten. Ob dies tatsächlich in allen Fällen auch der Realität entsprach, ist nicht überprüft worden. Van Walraven et al. (2002) stellten aber fest, dass im Falle von 792 Patienten nach Entlassung von einer internistischen Station bei im Mittel sechs hausärztlichen Konsultationen in 68\% der Fälle dem Hausarzt im gesamten Beobachtungszeitraum eines halben Jahres noch keine Entlassungsbriefe vorlagen. Bei nur $15 \%$ der Konsultationen lagen überhaupt Arztbriefe vor; Informationen über Diagnose und Therapieempfehlungen schon bei der ersten poststationären Konsultation standen dem Hausarzt nur in 8\% der Fälle zur Verfügung. Als Gründe wurden „noch nicht geschrieben“ (20\%) oder „noch nicht verschickt“ (51\%) angegeben. Diese Problematik und andere Kommunikations- und Abstimmungsschwierigkeiten könnten gerade an der Schnittstelle zwischen primärer und sekundärer Betreuung weitreichende Folgen, beispielsweise für die kurz-, oder längerfristige Verordnung von Medikamenten haben. So konnten Himmel et al. (1996) zeigen, dass sich selbst in der Langzeitmedikation ein „Turnover" von 50\% zwischen den Behandlungsetappen der Klinik und der hausärztlichen Betreuung ergibt. Rund ein Drittel aller Medikamente wurden im Krankenhaus abgesetzt oder ersetzt. Der Hausarzt seinerseits veränderte gleichermaßen die empfohlene Medikation nach Entlassung des Patienten aus dem Krankenhaus in einem Drittel der Fälle. 
Verständlicherweise können solche Verläufe auch in der vorliegenden Arbeit nicht ausgeschlossen werden. Nicht erklären lassen sich allerdings die isolierten schlechten Ergebnisse der ACE-Hemmer (Abschnitt 5.4, S.38). Im Falle von Schnittstellenproblemen hätten sich Einflüsse auf alle untersuchten Substanzen zeigen müssen.

Abschließend konnte trotz Abwägen aller erwähnten Fakten nicht plausibel geklärt werden, warum einzig und allein ACE-Hemmer in so niedrigem Maße von Hausärzten verschrieben wurden.

\subsubsection{Dosierung kardiovaskulärer Medikation im zeitlichen Verlauf}

Bezüglich der Verschreibung von nachweislich letalitätssenkenden Wirkstoffen wurde auch deren Dosierung untersucht (Abschnitt 5.5, S.40). Dies erschien zur Beurteilung der Behandlungsqualität bzw. der leitliniengerechten Behandlung als wichtig, da in nahezu allen klinischen Studien Dosis-Wirkungs-Beziehungen festgestellt werden konnten. Hierbei wurden Patienten, die ein Verum erhielten, meist in Niedrigdosis-, Mitteldosis- und Hochdosisgruppen unterteilt, um den Einfluss der Dosis auf den Grad der Letalitätsreduktion zu untersuchen. Daraus konnten für ACE-Hemmer und Beta-Blocker direkte Dosis-Wirkungs-Beziehungen abgeleitet werden; je höher die Dosis, desto ausgeprägter der Effekt. Exemplarisch seien hier zwei Studien zitiert. Packer et al. (1999) unterteilten im Rahmen einer kontrollierten Doppel-Blind-Studie 3164 Patienten mit Herzinsuffizienz (NYHA II-IV, EF<30\%) entweder in die Lisininopril-Niedrigdosis-Gruppe (bis $5 \mathrm{mg} / \mathrm{d}$ ) oder die Hochdosisgruppe (bis $35 \mathrm{mg} / \mathrm{d}$ ). Ein Vergleich beider Gruppen nach 58 Monaten zeigte eine signifikante Letalitätssenkung um $12 \%$ und eine um $24 \%$ geringere Hospitalisierungsrate zugunsten der Hochdosisgruppe.

Auch bei den Beta-Blockern stellt sich wahrscheinlich ein dosisabhängiger Effekt, besonders auf die Steigerung der Ejektionsfraktion, ein. Bristow et al. (1996) untersuchten in ähnlicher Weise 345 mit Carvedilol behandelte Patienten randomisiert unterteilt in Placebo-, Niedrigdosis- $(6,25 \mathrm{mg} / \mathrm{d})$, Mitteldosis- $(12,5 \mathrm{mg} / \mathrm{d})$ sowie Hochdosisgruppe (25 mg/d). Nach sechsmonatiger Beobachtung zeigte sich eine dosisabhängige Steigerung der Ejektionsfraktion sowie eine verringerte Letalität (Niedrigdosis: $6,0 \%$ vs. Hochdosis: 1,1\%) jeweils zugunsten der Hochdosisgruppe. 
Ergebnisse dieser Studien fanden in den Therapieempfehlungen der Leitlinien Eingang. So empfiehlt beispielsweise die Deutsche Gesellschaft für Kardiologie die aus den Letalitätsstudien abgeleiteten Zieldosierungen längerfristig anzustreben, da tendenziell eine niedrigere Gesamtletalität und eine signifikant geringere kombinierte kardiovaskuläre Letalität und Hospitalisationsrate bei gleicher Nebenwirkungshäufigkeit zu beobachten seien (Deutsche Gesellschaft für Kardiologie 2001, S.5). Hausärzten wird in diesem Zusammenhang von Seiten der Klinik angeraten, die Dosis neuangesetzter Medikamente schrittweise zu erhöhen. Besonders bei Beta-Blockern, aber auch bei ACE-Hemmern wird darauf hingewiesen, dass eine einschleichende Therapie und langsame Dosissteigerung zur Vermeidung von unerwünschten Wirkungen eminent wichtig seien (Karow, Lang-Roth 2005, S. 235, 240). Dieselben Empfehlungen sprechen auch die Leitlinien sowie die Arzneimittel-Fachinformation für diese Wirkstoffe aus.

In der vorliegenden Studie wurden neben den erwähnten Parametern auch die Einzeldosierungen der verschiedenen Medikamente (morgens, mittags, abends, zur Nacht) erfragt. Mithilfe der Einzeldosis der Präparate wurden anschließend die kumulativen Tagesdosen berechnet. Im Telefoninterview konnten Patienten aber mitunter nur lückenhafte Angaben zur derzeitigen Medikation machen, da z.B. aktuelle Medikamentenlisten des Hausarztes nicht auffindbar waren. Folglich standen für die Auswertung der Tagesdosen nicht von allen Patienten Daten zu Verfügung (Abschnitt 6.3.2, S.61). Dennoch sollten sich gemäß den Leitlinienempfehlungen in der poststationären Gruppe die Mehrzahl der Verordnungen von initial niedrigen bzw. mittleren Tagesdosen im Untersuchungszeitraum in den Hochdosisbereich hinein verschieben. Untersucht wurden als Grundpfeiler der evidenzbasierten Therapie Tagesdosen von ACE-Hemmern und Beta-Blockern. Die Referenzwerte wurden der Hausärztlichen Leitlinie Chronische Herzinsuffizienz (Leitliniengruppe Hessen 2003) entnommen.

Nach Auswertung der verschriebenen Tagesdosen (Tabelle 14, S.43) konnte gezeigt werden, dass sich insgesamt keine bedeutsamen Veränderungen in den Dosierungen der Wirkstoffe im Verlauf der Untersuchung ergaben. Schon zu Beginn der Untersuchung (Baseline-Erhebung) wurden ACE-Hemmer nur bei etwa der Hälfte der Patienten (poststationäre Gruppe 51\%, Kontrollgruppe 46\%) hochdosiert verordnet. 
Beta-Blocker wurden zu etwa einem Fünftel in beiden Gruppen hochdosiert verordnet. Die meisten Patienten nahmen Beta-Blocker im Bereich zwischen 25 und 75\% der Zieldosis ein (poststationäre Gruppe 55\%, Kontrollgruppe 65\%).

Nach Ablauf eines Jahres hatte eher eine Dosisreduktion als eine Dosissteigerung stattgefunden. Nur gut ein Drittel der poststationären Patienten (37\%) wurden in der Follow-Up-Erhebung noch hochdosiert mit ACE-Hemmern behandelt. Beta-Blocker wurden nahezu unverändert in etwa einem Fünftel der Fälle (poststationäre Patienten $20 \%$, Patienten der Kontrollgruppe15\%) hochdosiert verordnet.

Abschließend muss also vermutet werden, dass der Forderung nach einer weiteren ambulanten Dosissteigerung nach Entlassung sowie generell nach einer Hochdosistherapie in Anlehnung an die empfohlenen Zieldosierungen aus den Leitlinien seitens der Hausärzte scheinbar nicht nachgekommen wird. Es findet eher eine Dosisreduktion statt. Dieses Ergebnis bestätigt, dass sich die Empfehlungen offenbar international sowohl in der hausärztlichen wie auch in der stationären Betreuung nur schwer umsetzen lassen. Ähnliche Resultate lieferten verschiedene Versorgungsstudien. Beispielsweise sprechen Cleland et al. (2002) in der eingangs erwähnten Studie zur hausärztlichen Behandlung von 11062 herzinsuffizienten Patienten mit ACEHemmern und Beta-Blockern nur in ungefähr der Hälfte der Fälle von Dosierungen im Zielbereich nach den „Guidelines for the diagnosis and treatment of chronic heart failure“ der European Society of Cardiology (2001).

Die Hochdosistherapie selbst, deren Legitimation sich auf Letalitätsstudien für die entsprechenden Substanzen stützt, wird in verschiedenen Artikeln von Hausärzten wie Klinikärzten als größtenteils nicht umsetzbar beschrieben. Verständlich wird dies beispielsweise, wenn die empfohlenen Zieldosen mit den maximal zulässigen Tagesdosen verglichen werden (Karow, Lang-Roth 2005). Dabei fällt auf, dass diese in der Mehrzahl der Fälle identisch sind (Anhang A.2, S.78). Diese Tatsache birgt für den behandelnden Hausarzt einen therapeutischen Konflikt, da zum einen in pharmakologischer Literatur eher zur Kombination zweier Medikamente im Niedrig- oder Mitteldosisbereich geraten wird, um unerwünschte Arzneimittelwirkungen zu minimieren, als eine Substanz in ihrer Dosis auszureizen. Zudem haben die entsprechenden Substanzen eben nicht nur indirekte, prognostisch bedeutsame Wirkungen, sondern auch Wirkungen im direkten, eigentlichen Sinne, wie Blutdrucksenkung und Frequenzbegrenzung. Da es sich häufig bei kardiologisch/internistischen Patienten auch 
noch um eine Polypharmakotherapie handelt, ist bei stetiger Dosissteigerung an dieser Stelle schnell der körperliche und psychische Toleranzbereich des Patienten überschritten. Zudem könnte es als nicht plausibel erscheinen, Medikamente in ihrer Dosis weiter zu steigern, wenn sich bereits optimale, klinisch sichtbare Therapierfolge noch ohne unerwünschte Arzneimittel-Wirkungen eingestellt haben. Dass gerade Hausärzte vor allem symptomorientiert behandeln, zeigte eine Untersuchung von Chin et al. (1997), die einen Vergleich zwischen verschiedenen Fachgruppen hinsichtlich der Verschreibung der entscheidenden Substanzen durchführten. Kardiologen verschrieben dabei im Durchschnitt nicht nur mehr ACE-Hemmer bei asymptomatischer linksventrikulärer Dysfunktion (NYHA I) als Hausärzte (90\% vs. 55\%), sondern sie wählten auch höhere, adäquate Dosen. Aufschlussreiche Ergebnisse lieferte die Frage nach den Kriterien für die Festlegung der „richtigen“ Dosen. Hausärzte gaben hierbei an, ACE-Hemmer vor allem nach der Reduktion subjektiver Beschwerden der Patienten (45\%) oder klinischer Zeichen der Herzinsuffizienz (50\%) zu dosieren, während Kardiologen sich am häufigsten an aus klinischen Studien vorgegebenen Dosierungen (45\%), am Blutdruck (55\%) oder am Auftreten dosislimitierender Nebenwirkungen (38\%) orientierten.

Neben widersprüchlichen Richtlinien und symptomorientierter Therapie könnten auch Unterschiede im Fachwissen um die verschiedenen Erkrankungen und entsprechenden Therapieempfehlungen ursächlich sein. So stellte sich beispielsweise bei einer Befragung von 116 polnischen Hausärzten heraus, dass diese zwar in über der Hälfte der Fälle die korrekte Initialdosis von Enalapril benennen konnten, aber nur 3\% von ihnen kannten die empfohlene Zieldosis der europäischen Leitlinien (Wierzchowiecki et al. 2005).

Abschließend ist anzumerken, dass die Legitimation der Hochdosistherapie derweil selbst schon wieder auf dem Prüfstand steht. Neuere Arbeiten verweisen zunehmend auf Untersuchungen, nach denen höchste Dosierungen nicht nur klinisch nicht praktikabel wären, sondern außerdem keinen zusätzlich positiven Einfluss auf das Überleben der Patienten hätten. So kommt beispielsweise Roffmann (2004) nach Auswertung von Artikeln zu Dosis-Wirkungs-Beziehungen zwischen 1987 und 2002 zu dem Schluss, dass wohl Surrogatparameter wie NYHA-Stadien oder Hospitalisierungsraten durch höhere Dosen reduziert würden, aber eine dosisabhängige Verlängerung des Überlebens nicht überzeugend bewiesen werden könnte. Und schließ- 
lich, so Roffmann, hätten Patienten mit niedrig dosierter Therapie immer noch ein besseres „Outcome“ als Patienten ohne Therapie (Roffmann 2004). 


\subsection{Methodische Diskussion der Ergebnisse}

\subsubsection{Repräsentativität und Vergleichbarkeit der untersuchten Pa- tientengruppen}

Entscheidend für die Übertragbarkeit der Ergebnisse der vorliegenden Studie ist die Repräsentativität der Stichprobe. Die untersuchten Patienten entsprachen in Alter und (ursächlichen) Begleiterkrankungen typischen Patienten mit Herzinsuffizienz (Abschnitt 5.2, S.31). Die große Mehrheit der Teilnehmer gehörten der Altersgruppe mit der höchsten Inzidenz und Prävalenz an, d.h. der 60 bis 80-Jährigen. Die Verteilung der Komorbiditäten ist nicht nur charakteristisch, sondern entspricht auch den häufigsten, einer Herzinsuffizienz zugrunde liegenden Erkrankungen (Alexander et al. 1999, S. 1070ff.).

Laut der Framingham-Studie (Ho et al. 1993) liegen die Ursachen in über der Hälfte der Fälle in einer koronaren Herzkrankheit und/oder in einem arteriellen Hypertonus begründet. Wilhelmsen et al. (2001) fanden bei 937 Patienten mit Herzinsuffizienz als Ursachen sogar in 58,8\% der Fälle eine KHK mit oder ohne Hypertension und in 20,3\% der Fälle einen Hypertonus allein. Erkrankungen wie Diabetes mellitus (4,5\% der Fälle) oder Herzklappenerkrankungen (5,8\% der Fälle) spielten eher eine untergeordnete Rolle. In beiden Kohorten finden sich als häufigste Nebendiagnosen ebenfalls KHK (53\% vs. $39 \%$ ) sowie arterielle Hypertonie (57\% vs. $67 \%$ ); auch ein Diabetes mellitus war bei einem Drittel der Patienten dokumentiert. Erkrankungen wie chronisch obstruktive Lungenerkrankungen oder kompensierte Niereninsuffizienz waren, auch im Hinblick auf etwaige Kontraindikationen für die untersuchten Wirkstoffe, selten.

Für das gewählte Kontrollgruppendesign war die Vergleichbarkeit der beiden Patientengruppen entscheidend. Der Vergleich der Kohorten bezüglich entscheidender soziodemographischer und klinischer Daten zeigte, dass diese Forderungen bezüglich Alter, Begleiterkrankungen und Schwere der Erkrankung erfüllt wurden (Tabelle 9, S.32). Einzig die stationäre Behandlung in der Vorgeschichte sollte die Gruppen unterscheiden. Bei der Untersuchung der Klinikaufenthalte im Untersuchungszeitraum konnte gezeigt werden, dass im Vergleich zur poststationären Kohorte Patienten der Kontrollgruppe im Untersuchungszeitraum fast nie stationär behandelt wurden (40\% vs. 7\%), (Abschnitt 5.6, S.43). Es kann also davon ausgegangen werden, dass die 
ausgewählten Patienten in den einzelnen Gruppen erstens repräsentativ für die untersuchte Erkrankung und zweitens miteinander vergleichbar waren.

\subsubsection{Güte der eingesetzten Erhebungsinstrumente}

Die Durchführung der Befragung mittels eines Fragebogens und insbesondere durch ein Telefoninterview erwies sich teilweise als schwierig. Patienten oder deren Angehörige reagierten mit großer Skepsis auf die Anrufer. Trotz vorgegebener, strukturierter Vorgehensweise mit Nennung des Namens, der Abteilung, des Studiengegenstandes sowie der kurzen Erinnerung an vorausgegangene Telefonate wollten sich einige Teilnehmer gegenüber dem Anrufer nicht äußern (Abschnitt 5.1, S.30). Verständlich erscheint diese Reaktion angesichts der zunehmenden kommerziellen Werbung älterer Kunden am Telefon seitens der Industrie. Teilweise gelang es, die Patienten wenigstens zum Ausfüllen und Zurücksenden des Fragebogens zu überreden, um zumindest einen Teil der Daten für Teilstudien verwenden zu können. Ferner waren zahlreiche Patienten zum Zeitpunkt der Konsultation persönlich verhindert. In diesem Falle wurde ein unverbindlicher Termin für einen erneuten Anruf mit den Patienten vereinbart. Auch hierbei kam es mitunter zu Problemen, da Patienten erneut nicht erreichbar waren.

Auch die inhaltliche Datenerhebung selbst gestaltete sich teilweise problematisch. Da es sich z.B. bei der Erhebung der Medikation der Patienten um eine telefonische Befragung handelte, waren die Angaben teilweise schwer zu erheben bzw. unvollständig. Die Mitarbeit des einzelnen Patienten sowie die Verfügbarkeit des Ehepartners als Informationsquelle oder der aktuellen Medikamentenliste des Hausarztes bestimmten maßgeblich die Fülle der erhebbaren Datenmenge. Folglich standen für die Auswertung der Tagesdosen nicht von allen Patienten Daten zu Verfügung. In einigen Fällen musste von den festgelegten Frageprotokollen unter Inkaufnahme von ungewollten Suggestionen abgewichen werden, um dem Erklärungsbedarf der Patienten zu entsprechen. Weiterhin stellte sich ein Zeitraum von zwölf Monaten in Einzelfällen als zu lang heraus, um sich an spezielle Begebenheiten wie beispielsweise Krankenhausaufenthalte oder die Gründe für Veränderungen in der Medikation erinnern zu können. Insgesamt kam es aufgrund dieser Schwierigkeiten zu einem gewissen Verlust an Informationen, woraus, wie erwähnt, niedrige auswertbare Fall- 
zahlen, wie z.B. im Falle der Erhebung der Dosierungen der Medikamente (Abschnitt 5.5, S.41), resultierten.

Dennoch erwies sich das Telefoninterview als sinnvolles Instrument, da von vornherein Unsicherheiten seitens der Patienten direkt durch den Interviewer beseitigt werden konnten und die Responderquote sehr hoch war (poststationäre Kohorte: 83\%, Kontrollgruppe: 98\%), (Tabelle 8, S.30).

Der im Anschluss an die telefonische Befragung an die Patienten verschickte Fragebogen sollte dem Zweck dienen, durch eine Selbsteinschätzung des Patienten in etwa die Schwere der Erkrankung beurteilen zu können. Hierbei wurden dem Patienten einerseits typische Fragen zur alltäglichen Belastbarkeit im Sinne der NYHA-Stadien gestellt. Zusätzlich wurden Fragen zur Einteilung der Schwere der Erkrankung gestellt, die in Form eines Entscheidungsbaumes zu beantworten waren. In beiden Fällen, aber besonders bei der Bearbeitung der Fragen in Form eines Entscheidungsbaumes traten Probleme auf. Patienten schätzten die Fragen zum Teil falsch ein. Fragen, die auf eine kardial bedingte begrenzte Belastbarkeit abzielten, wurden augenscheinlich auf andere Erkrankungen, wie z.B. Erkrankungen des Bewegungsapparates (z.B. rheumatoide Arthritis) oder auf chronische Lungenerkrankungen (z.B. COPD) übertragen. Darüber hinaus ergaben sich rein technische Schwierigkeiten, wenn entlang des Entscheidungsbaumes seitens der Patienten nicht differenziert vorgegangen wurde, sondern schlichtweg alle Fragen mit Antwortkreuzen versehen wurden. Durch diese Schwierigkeiten bedingt kam es wiederum in Einzelfällen einerseits zu unterschiedlichen Ergebnissen in der Bestimmung der Schwere der Erkrankung nach den unterschiedlichen Methoden und andererseits insgesamt zu einer verminderten auswertbaren Datenmenge.

Im weiteren Verlauf wurde die Versendung eines Verordnungsplans mit dem Fragebogen als sinnvoll erachtet. Die Patienten wurden erneut gebeten, ihre Medikation nach einem vorbeschriebenen Beispiel vollständig anzugeben. Dadurch konnten die Patienten noch einmal in Ruhe ihre aktuelle Medikation ausführen bzw. den Ehepartner oder andere Angehörige befragen, die durchaus häufig mit der Verantwortung für die Medikamenteneinnahme betraut waren. Im Folgenden wurden die Angaben aus dem Telefoninterview mit denen des zurückgesandten Verordnungsplans abgeglichen. So konnten einerseits fehlende Werte (z.B. Dosierungen) ergänzt und andererseits aber auch insgesamt eine größere Zuverlässigkeit dieser entscheidenden, im Telefoninterview erhobenen Daten erreicht werden. 


\subsubsection{Limitationen des Untersuchungsdesigns}

Problematisch erscheint die Beurteilung der Behandlungsgüte vor dem Hintergrund, dass es sich bei den durchgeführten Erhebungen trotz des Längsschnittdesigns letztendlich um Momentaufnahmen handelte. Trotz einer Fülle anamnestischer Daten zum Krankheitsverlauf zwischen zwei Erhebungen konnte die Frage nach den Ereignissen mit Einfluss auf die zum Erhebungszeitpunkt aktuelle Therapie nicht erschöpfend geklärt werden. So bestanden beispielsweise zwar Informationen über Krankenhausaufenthalte und die grobe Einschätzung der Maßnahmen (Abschnitt 5.6, S.43), jedoch fehlten mit den Entlassungsbriefen der betreffenden Kliniken die jeweiligen Therapieempfehlungen. Andererseits sollte es im Ergebnis häufigerer Hospitalisierungen eher zu einer weiter intensivierten Therapie als zum Absetzen der „FirstLine-Medikamente" kommen, da die medikamentöse Versorgung von herzinsuffizienten Patienten in der Klinik doch im Allgemeinen bessere Qualität erreicht als eine rein hausärztliche Betreuung. Daten sowohl der vorliegenden Arbeit (Abschnitt 5.4, S.38), wie auch anderer Studien (Anhang A.1, S.78) zur Untermauerung dieser Aussage wurden bereits beschrieben. So berichteten beispielsweise Komajda et al. (2003) von Verschreibungsraten von $80 \%$ für ACE-Hemmer im stationären Bereich im Vergleich zu den von uns untersuchten Verordnungshäufigkeiten in der Entlassungsmedikation von $75 \%$ der Patienten. Cleland et al. fanden bei einem primärärztlich versorgten Kollektiv bei $60 \%$ der Patienten eine Verschreibung von ACE-Hemmern und bei $34 \%$ von innen eine Behandlung mit Beta-Blockern, was unsere Ergebnisse widerspiegeln (hausärztlich versorgte Kontrollgruppe; ACE-Hemmer: 58\%, Beta-Blocker: $42 \%)$. Somit bieten die hier dargestellten Ergebnisse zu Klinikaufenthalten während des Untersuchungszeitraums keinen weiteren Anhalt für die niedrigen Verschreibungsraten von ACE-Hemmern (Follow-Up-Erhebung der poststationären Kohorte), die als universelles Qualitätskriterium in der Behandlung der chronischen Herzinsuffizienz anzusehen sind.

Diskutiert werden muss auch die Frage, inwiefern die dargestellten Ergebnisse auf verschiedene "Cluster" von Hausarztpraxen zurückzuführen sein könnten. Gibt es also „leitlinienadhärent“ und „nicht leitlinienadhärent behandelnde“ Praxen und gab es in der vorliegenden Untersuchung von der einen Sorte mehr als von der anderen? 
Die Patienten der poststationären Kohorte wurden nicht aus einer Anzahl von ausgewählten Praxen, sondern direkt in Rahmen ihres stationären Aufenthaltes rekrutiert. Somit handelte es sich um Patienten aus dem gesamten Einzugsgebiet der Klinik (Großraum Südniedersachsen, Einzugsradius ca. 80 km). Dies führte im Nachhinein zu einer Relation Patienten/Praxen von annähernd eins zu eins (Tabelle 8, S.30). Cluster-Effekte können demnach ausgeschlossen werden.

\subsection{Bewertung von Leitlinien-Empfehlungen als "Goldstandard“ in der Pharmakotherapie primärärztlich versorgter Patienten}

Als primäre Bezugsquelle sowohl für die Therapie verschiedener Erkrankungen an sich, aber auch zur Qualitätsbeurteilung haben sich im Ausland frühzeitig Leitlinien etabliert. Insbesondere in den USA und in Großbritannien haben Leitlinien eine hohe gesundheitspolitische Bedeutung, da sich mit ihnen die Erwartung verbindet, zu wissenschaftlich begründeten und vor allem ökonomisch angemessenen Entscheidungen zu gelangen. Auch die Qualitätsschwankungen in der primärärztlichen Therapie sollen begrenzt werden (Kiewel 2002, S.33f.). Für die Behandlung der chronischen Herzinsuffizienz bestehen in Deutschland bereits zahlreiche Auflagen von Leitlinien unterschiedlicher Gesellschaften.

In der vorliegenden Untersuchung konnten Missstände in der hausärztlichen Versorgung bzw. eine wenig leitliniengerechte Therapie der chronischen Herzinsuffizienz beschrieben werden. Mögliche Gründe für diese Fakten bezüglich der Verwendung von Leitlinien müssen auf mehreren Ebenen diskutiert werden.

Die Praktikabilität von Leitlinienempfehlungen im hausärztlichen Alltag ist verständlicherweise kritisch zu betrachten. In einer Untersuchung zur Umsetzung von Leitlinien in der primären Versorgung von Patienten wurden 151 Ärzte vor Beginn der Erhebung zu ihrer Einstellung gegenüber Leitlinien als solchen und deren Praktikabilität sowie zu deren Inhalt befragt. Obwohl die Mehrheit der Befragten die Leitlinie als hilfreiches Werkzeug zur Verbesserung der Versorgung ansah (zutreffend: 64\%, absolut zutreffend: $19 \%$ ), empfanden viele Ärzte die Vorschläge als zu sehr vereinfacht (zutreffend: $53 \%$ ) und zu starr für die individuelle Behandlung von Patienten (neutral: 29\% zutreffend: $23 \%$ ) (Tierney et al. 2003). 
Eine gute Möglichkeit, diese Problematik zu minimieren bestünde darin, engagierte, praktizierende Hausärzte selbst mit in die Erarbeitung von Leitlinien einzubeziehen. Durch die Leitliniengruppe Hessen (2003) sind diese Vorschläge teilweise umgesetzt worden. Bei den Mitgliedern der Leitliniengruppe handelt es sich um praktizierende Ärzte der Kassenärztlichen Vereinigung Hessen, die im Rahmen der hausärztlichen Qualitätssicherung langjährig in Pharmakotherapiezirkeln tätig sind. Im Ergebnis dieser Zusammenarbeit konnten Leitlinien vorgelegt werden, die sich beispielsweise nicht mehr an der stadiengemäßen Behandlung nach der NYHA-Einteilung orientieren, sondern vielmehr einen symptomorientierten Entscheidungsbaum präsentieren (Anhang A.4, S.80). Diese Empfehlungen weichen zwar, streng genommen, von den Empfehlungen internationaler Leitlinien und denen der Deutschen Gesellschaft für Kardiologie (2001) geringfügig ab, sie haben jedoch ein größeres Maß an Praktikabilität gewonnen. Hausärzte könnten sich dadurch vermehrt angesprochen fühlen, häufiger Leitlinien zu Rate zu ziehen, was eine steigende Quote leitliniengerechter und damit besser behandelter Patienten zur Folge hätte.

Auch die Deutsche Gesellschaft für Allgemeinmedizin (DEGAM) ist mit der Erarbeitung einer hausärztlich orientierten Leitlinie beschäftigt, die sich gegenwärtig noch im Praxistest befindet. Die Leitlinie erhebt den Autoren zufolge den Anspruch, als einzige Leitlinie direkt an Hausärzte gerichtet und gleichzeitig evidenzbasiert, interdisziplinär konsentiert und in der hausärztlichen Praxis getestet zu sein. Hieraus könnten sich eine noch bessere und breitere Anwendbarkeit durch Hausärzte ergeben.

Die Frage danach, was Leitlinien aber tatsächlich leisten können und wo die Grenzen von Leitlinien zu sehen sind, verändert sich durch all diese Maßnahmen nicht. Sie sind und bleiben nur „[...] Orientierungs- und Entscheidungshilfen [...] für typische Beschwerdebilder und Behandlungssituationen - für den Normalfall." (Leitliniengruppe Hessen 2003, S.3). Die individuelle Konstellation ist letztendlich nur für den behandelnden Arzt einsehbar und somit liegt die Therapieentscheidung in seinen Händen. Aber gerade in der heutigen Zeit, wo niedergelassene Ärzte z.B. leicht in Versuchung geraten, von Pharmafirmen unterstützte Studien als allgemein anerkannten Konsens zu verstehen, können Leitlinien ein effektives, unabhängiges, einfach und kostenlos zu beziehendes Weiterbildungsorgan sein. Die kontinuierliche Überarbeitung sichert zudem die Aktualität der Empfehlungen auf dem neuesten Stand der Erkenntnisse. Regelmäßige Neuauflagen der Leitlinien könnten aber auch Segen und Fluch zugleich sein. Einerseits könnte sich der praktizierende Hausarzt motiviert 
fühlen, seine Patienten stets nach dem neuesten Stand der Forschung zu versorgen. Andererseits zeigen die Revisionen häufig aber auch, dass die Empfehlung einer zuvor als sicher wirksam oder sicher anzuwendenden Therapie wieder zurückgenommen wird. Diese Umstände könnten durchaus den Glauben an eine evidenzbasierte Medizin und die daraus abgeleiteten Therapien weiter schmälern.

\subsection{Schlussfolgerung und Ausblick}

Im Rahmen der vorliegenden Arbeit konnte gezeigt werden, dass es bei Patienten mit Herzinsuffizienz, die nach krankheitsspezifischer stationärer Behandlung durch den Hausarzt weiterbetreut wurden, zu gesteigerten Verschreibungsraten in der Therapie der Herzinsuffizienz entscheidender Medikamente kam. Hierbei handelte es sich sowohl um Wirkstoffe mit Verbesserung der Prognose sowie um rein symptomatisch wirksame Substanzen. Zudem blieben die Verordnungshäufigkeiten über den Beobachtungszeitraum von einem Jahr weitestgehend konstant und unterschieden sich damit deutlich von Patienten einer rein hausärztlich betreuten Kontrollgruppe. Einzige Ausnahme blieben ACE-Hemmer, die nach anfänglich hohen Verschreibungszahlen in der Klinik im Untersuchungszeitraum auf das Niveau der Kontrollgruppe zurückgingen. Die stationäre Sicherung der Diagnose „Herzinsuffizienz“ sowie Empfehlungen zur weiteren therapeutischen Vorgehensweise scheinen demnach einen positiven Einfluss auf die langfristige Verordnung von therapeutisch entscheidenden Substanzen durch den Hausarzt gehabt zu haben. Lediglich ACE-Hemmer blieben von diesem Trend ausgenommen.

Nicht zufriedenstellend geklärt werden konnte die Frage, warum eine Behandlung mit ACE-Hemmern den Hausarzt vor Probleme stellt. Die Daten der vorliegenden Arbeit konnten keine hinreichenden Ergebnisse zu den Gründen des Verschreibungsverhaltens der Hausärzte liefern. Eventuell besteht hier weiter Aufklärungsbedarf. Weiterhin stellt sich die Frage, ob höhere Verordnungsraten der in der Behandlung der Herzinsuffizienz eingesetzten Medikamente tatsächlich zu einer verminderten Letalität eines hausärztlich betreuten Patientenkollektivs führen; auch diese Frage konnte anhand der Datenlage nicht beantwortet werden. 
Im Ergebnis der vorliegenden Arbeit könnte Hausärzten empfohlen werden, die Verantwortung für die optimale Versorgung eines Patienten mit Herzinsuffizienz mit (beispielsweise auch niedergelassenen) Fachkollegen zu teilen. Eine regelmäßige Konsultation mit gelegentlicher Prüfung der Diagnose und ständiger Optimierung der Therapie sowie anschließend eine gute Kommunikation zwischen den Kollegen wären erforderlich, um den Patienten optimal versorgen zu können. Dabei muss aber weiterhin die Koordination der Maßnahmen um das Management des Patienten unter der Leitung des Hausarztes stehen. Zur Verbesserung der Qualität der hausärztlichen Versorgung müssen den Ärzten weitere sinnvolle und praxisorientierte Hilfsmittel zur Verfügung stehen. Ein guter Ansatz wären Leitlinien, die noch stärker mit symptomorientierten Algorithmen arbeiten. Denkbar wären auch Qualitätszirkel bzw. „Peer-Fortbildungen“, in denen „schwierige“ Patienten, in Anlehnung an die BalintGruppen im Bereich der Psychosomatik, v.a. mit hausärztlichen Kollegen aber auch mit Spezialisten besprochen werden könnten.

Studien mit größeren Fallzahlen und vollständigen Daten aus größeren Einzugsbereichen wären zur Prüfung der gefundenen Ergebnisse äußerst wertvoll. 


\section{$7 \quad$ Zusammenfassung}

Ausgangspunkt. Die medikamentöse Versorgung von Patienten mit chronischer Herzinsuffizienz durch den Hausarzt ist nicht zufriedenstellend. Als Gründe werden unter anderem Unsicherheiten bezüglich der Sicherung der Diagnose sowie der Einleitung einer spezifischen Therapie genannt. Die vorliegende Untersuchung sollte klären, ob die hausärztliche Versorgung durch Diagnosesicherung und Therapieempfehlung aus der Klinik dauerhaft verbessert werden kann.

Methode. Hausärztlich betreute Patienten mit Herzinsuffizienz wurden hinsichtlich ihrer krankheitsspezifischen Medikation im Verlauf eines Jahres untersucht. Dabei wurden die Patienten der poststationären Gruppe initial mit gesicherter Diagnose und Therapieempfehlung aus einer Universitätsklinik entlassen, während die Patienten der Kontrollgruppe ausschließlich durch den Hausarzt betreut wurden. Als Datenquelle dienten die Entlassungsbriefe bzw. die hausärztliche Routinedokumentation. Zudem wurden Telefoninterviews durchgeführt und Fragebögen versendet.

Ergebnisse. In beiden Gruppen nahmen jeweils 88 Patienten zum ersten Untersuchungszeitpunkt an der Studie teil. Zur Zwölf-Monats-Follow-Up-Erhebnung lagen die Daten von 73 Patienten (poststationäre Gruppe) bzw. 86 Patienten (Kontrollgruppe) vor. Zum Zeitpunkt der Entlassung aus der Klinik nahmen signifikant mehr Patienten der poststationären Gruppe ACE-Hemmer (75\% vs. $58 \% ; \chi^{2}=6,474, p=0,011$ ), BetaBlocker ( $89 \%$ vs. $\left.50 \% ; \chi^{2}=28,800, p<0,001\right)$ und Diuretika $\left(90 \%\right.$ vs. $68 \% ; \chi^{2}=6,129$, $p<0,001)$ ein als Patienten der Kontrollgruppe. Nach Ablauf eines Jahres konnten bei konstanten Verschreibungszahlen in der Kontrollgruppe weiterhin signifikante Unterschiede zugunsten der poststationären Gruppe für Beta-Blocker (77\% vs. 52\%; $\left.\chi^{2}=10,134, p=0,001\right)$ und Diuretika (86\% vs. $\left.63 \% ; \chi^{2}=6,244, p=0,002\right)$ festgestellt werden. Für ACE-Hemmer konnte dieser Unterschied nach einem Jahr nicht mehr nachgewiesen werden (poststationäre Gruppe: 59\% vs. Kontrollgruppe: 62\%; $\left.\chi^{2}=0,122, p=0,726\right)$. Auch bei der Beachtung der Verschreibung entweder eines ACE-Hemmers oder alternativ eines AT1-Blockers waren die Unterschiede zwischen den Gruppen nach einem Jahr nicht mehr nachweisbar (poststationäre Gruppe: 75\% vs. Kontrollgruppe: $\left.74 \% ; \chi^{2}=0,018, p=0,894\right)$. In $53 \%$ der Fälle von abgesetzten ACE-Hemmern bestanden keine plausiblen Gründe. Insgesamt wurden die progno- 
severbessernden Medikamente nur in ungenügendem Maße im Bereich der empfohlenen Zieldosen verordnet. Eine Steigerung der Dosen im Verlauf der poststationären Weiterbetreuung fand nicht statt; eher konnte eine Dosisreduktion beobachtet werden. Eine Hochdosistherapie mit Beta-Blockern erfolgte in je 21\% der Fälle bei Entlassung und nach einem Jahr, ACE-Hemmer wurden zu 51\% bzw. nur noch zu 37\% nach einem Jahr hochdosiert verordnet.

Schlussfolgerung. Durch Diagnosesicherung und Therapieempfehlungen lassen sich dauerhaft höhere Verschreibungsraten von prognose- und symptombessernden Medikamenten in der hausärztlichen Versorgung von Patienten mit Herzinsuffizienz erzielen. Lediglich die Therapie mit ACE-Hemmern stellt eine Ausnahme dar. Gründe hierfür müssen weitergehende Studien klären. 


\section{Literaturverzeichnis}

ACC/AHA Guidelines for the Evaluation and Management of Chronic Heart Failure in the Adult (2001)

(http://www.acc.org/clinical/guidelines/failure/pdfs/hf_fulltext.pdf; Stand: 27.07.2006)

AHCPR (Agency for Health Care Policy and Research. US Department of Health and Human Services.): Acute pain management: operative and medical procedures and trauma. Clinical practice guideline No. 1 (1993)

(http://www.ahrq.gov/; Stand: 27.07.2006)

Ahmed A, Allman RM, Kiefe CL, Person SD, Shaneyfelt TM, Sims RV, Howard G DeLong JF (2003): Association of consultation between generalists and cardiologists with quality and outcomes of heart failure care. Am Heart J; 145:1086-1093.

Alexander K, Daniel WG, Diener HC, Freund M, Köhler H, Matern S, Maurer HH, Michel BA, Nowak D, Risler T, Schaffner A, Scherbaum WA, Sybrecht GW, Wolfram G, Zeitz M: Thiemes Innere Medizin. Georg Thieme Verlag, Stuttgart 1999

Arzneimittelkommission der deutschen Ärzteschaft: Chronische Herzinsuffizienz (2001)

(http://www.akdae.de/35/10Hefte/88_Herzinsuffizenz_2001_2Auflage.pdf; Stand: 27.07.2006)

Barat I, Andreasen F, Damsgaard EM (2001): Drug therapy in the elderly: what doctors believe and patients actually do. Br J Clin Pharmacol; 51:615-622.

Boyles PJ, Peterson GM, Bleasel MD, Vial JH (2004): Undertreatment of congestive heart failure in an Australian setting. J Clin Pharm Ther; 29:15-22.

Bristow MR, Gilbert EM, Abraham WT, Adams KF, Fowler MB, Hershberger RE, Kubo SH, Narahara KA, Ingersoll H, Krueger S, Young S, Shusterman N (1996): Carvedilol produces dose-related improvements in left ventricular function and sur- 
vival in subjects with chronic heart failure. MOCHA Investigators. Circulation; 94:2807-2816.

Buddeberg C, Willi J: Psychosoziale Medizin. 2.Auflage; Springer-Verlag, Berlin 1998

Bundesverband der Pharmazeutischen Industrie: Service GmbH FachInfo-Service (2006)

(www.fachinfo.de; Stand: 27.07.2006)

Butler J, Arbogast PG, Daugherty J, Jain MK, Ray WA, Griffin MR (2004): Outpatient utilization of angiotensin-converting enzyme inhibitors among heart failure patients after hospital discharge. J Am Coll Cardiol; 43:2036-2043.

Chin MH, Friedmann PD, Cassel CK, Lang RM (1997): Differences in generalist and specialist physicians' knowledge and use of angiotensin-converting enzyme inhibitors for congestive heart failure. J Gen Intern Med; 12:523-530.

CIBIS-II Investigators and Committees (1999): The Cardiac Insufficiency Bisoprolol Study (CIBIS-II): a randomised trial. Lancet; 353:9-13.

Cleland JG, Cohen-Solal A, Aguilar JC, Dietz R, Eastaugh J, Follath F, Freemantle N, Gavazzi A, van Gilst WH, Hobbs FD, Korewicki J, Madeira HC, Preda I, Swedberg K, Widimsky J; IMPROVEMENT of Heart Failure Programme Committees and Investigators. Improvement programme in evaluation and management; Study Group on Diagnosis of the Working Group on Heart Failure of The European Society of Cardiology (2002): Management of heart failure in primary care (the IMPROVEMENT of Heart Failure Programme): an international survey. Lancet; $\underline{360}: 1631-1639$.

Cline CM, Bjorck-Linne AK, Israelsson BY, Willenheimer RB, Erhardt LR (1999): Non-compliance and knowledge of prescribed medication in elderly patients with heart failure. Eur J Heart Fail; 1:145-149.

D'Alto M, Pacileo G, Calabro R (2003): Nonpharmacologic care of heart failure: patient, family, and hospital organization. Am J Cardiol; 91:51-54. 
Deutsche Gesellschaft für Kardiologie: Leitlinien zur Therapie der chronischen Herzinsuffizienz (2001)

(http://www.dgkardio.de/Leitlinien/LeitlinienHerzinsuffizienz.pdf; Stand: 27.07.2006)

European Society of Cardiology: Guidelines for the diagnosis and treatment of chronic heart failure (2001)

(http://www.escardio.org/NR/rdonlyres/83B0E854-D56A-47C1-

988F585F4EBFEAF8/0/CHF_diagnosis.pdf; Stand: 27.07.2006)

Flather MD, Shibata MC, Coats AJ, Van Veldhuisen DJ, Parkhomenko A, Borbola J, Cohen-Solal A, Dumitrascu D, Ferrari R, Lechat P, Soler-Soler J, Tavazzi L, Spinarova L, Toman J, Bohm M, Anker SD, Thompson SG, Poole-Wilson PA; SENIORS Investigators (2005): Randomized trial to determine the effect of nebivolol on mortality and cardiovascular hospital admission in elderly patients with heart failure (SENIORS). Eur Heart J; 26:215-225.

Fricke U, Günther J, Zawinell A: Methodik der ATC-Klassifikation und DDDFestlegung für den deutschen Arzneimittelmarkt. 3. Auflage, Wissenschaftliches Institut der AOK (WIDO), Bonn 2004

Himmel W, Tabache M, Kochen MM (1996): What happens to long-term medication when general practice patients are referred to hospital? Eur J Clin Pharmacol; 50:253-257.

Ho KK, Pinsky JL, Kannel WB, Levy D (1993): The epidemiology of heart failure: the Framingham Study. J Am Coll Cardiol; 22:6-13.

Hobbs FD, Jones MI, Allan TF, Wilson S, Tobias R (2000): European survey of primary care physician perceptions on heart failure diagnosis and management (EuroHF). Eur Heart J; 21:1877-1887.

Karow T, Lang-Roth R: Allgemeine und spezielle Pharmakologie und Toxikologie. 13.Auflage; Selbstverlag, Köln 2005

Kiewel A: Nehmen Sie ihre Medikamente selbst? Juventa Verlag, München 2002 
Komajda M, Bouhour JB, Amouyel P, Delahaye F, Vicaut E, Croce I, Rougemond E, Vuittenez F, Leutenegger E (2001): Ambulatory heart failure management in private practice in France. Eur J Heart Fail; $\underline{3}: 503-507$.

Komajda M, Follath F, Swedberg K, Cleland J, Aguilar JC, Cohen-Solal A, Dietz R, Gavazzi A, Van Gilst WH, Hobbs R, Korewicki J, Madeira HC, Moiseyev VS, Preda I, Widimsky J, Freemantle N, Eastaugh J, Mason J; Study Group on Diagnosis of the Working Group on Heart Failure of the European Society of Cardiology (2003): The EuroHeart Failure Survey Programme - a survey on the quality of care among patients with heart failure in Europe. Part 2: treatment. Eur Heart J; 24:464-474.

Kompetenznetz Herzinsuffizienz: Optimierung der ambulanten Therapie bei Herzinsuffizienz (2006)

(http://www.allgemeinmedizin.uni-frankfurt.de/forschung/herzinsuffizienz.html; Stand: 19.07.2006)

Krum H (2004): Tolerability of carvedilol in heart failure: clinical trials experience. Am J Cardiol; 93:58-63.

Leitliniengruppe Hessen: Hausärztliche Leitlinie Chronische Herzinsuffizienz (2003) (http://www.leitlinien.de/leitlinieanbieter/deutsch/pdf/hessenhireport;

Stand: 27.07 .2006$)$

Lown B: Die verlorene Kunst des Heilens. Schattauer Verlag, Frankfurt am Main 2002

McKelvie RS, Yusuf S, Pericak D, Avezum A, Burns RJ, Probstfield J, Tsuyuki RT, White M, Rouleau J, Latini R, Maggioni A, Young J, Pogue J (1999): Comparison of candesartan, enalapril, and their combination in congestive heart failure: randomized evaluation of strategies for left ventricular dysfunction (RESOLVD) pilot study. The RESOLVD Pilot Study Investigators. Circulation; 100:1056-1064. 
McMurray JJ (1996): Concise Guide to the Management of Heart Failure - World Health Organization/Council on Geriatric Cardiology Task Force on Heart Failure Education. Am J Geriatr Cardiol; $\underline{5}: 13-30$.

McMurray JJ (1998): Failure to practice evidence-based medicine: why do physicians not treat patients with heart failure with angiotensin-converting enzyme inhibitors? Eur Heart J; Suppl L:15-21.

MERIT-HF Study Group (1999): Effect of metoprolol CR/XL in chronic heart failure: Metoprolol CR/XL Randomised Intervention Trial in Congestive Heart Failure (MERIT-HF). Lancet; 353:2001-2007.

Murray-Thomas T, Cowie MR (2003): Epidemiology and clinical aspects of congestive heart failure. J Renin Angiotensin Aldosterone Syst; 4:131-136.

National Institute for Clinical Exellence: Management of chronic heart failure in adults in primary and secondary care (2003) (http://www.nice.org.uk/pdf/CG5NICEguideline.pdf; Stand: 27.07.2006)

Packer M, Poole-Wilson PA, Armstrong PW, Cleland JG, Horowitz JD, Massie BM, Ryden L, Thygesen K, Uretsky BF (1999): Comparative effects of low and high doses of the angiotensin-converting enzyme inhibitor, lisinopril, on morbidity and mortality in chronic heart failure. ATLAS Study Group. Circulation; 100:2312-2318.

Pfeffer MA, Braunwald E, Move LA, Basta L, Brown EJ, Cuddy TE, Davis BR, Geltman EM, Goldman S, Flaker GC (1992): Effect of captopril on mortality in patients with left ventricular dysfunction after myocardial infarction. Results of the survival and ventricular enlargement trial. The SAVE investigators. N Engl J Med; 327:669-677.

Pflugfelder PW, Baird MG, Tonkon MJ, DiBianco R, Pitt B (1993): Clinical consequences of angiotensin-converting enzyme inhibitor withdrawal inchronic heart failure: a double-blind, placebo-controlled study of quinapril. The Quinapril Heart Failure Trial Investigators. J Am Coll Cardiol; 22:1557-1563. 
Pitt B, Segal R, Martinez FA, Meurers G, Cowley AJ, Thomas I, Deedwania PC, Ney DE, Snavely DB, Chang PI (1997): Randomised trial of losartan versus captopril in patients over 65 with heart failure (Evaluation of Losartan in the Elderly Study, ELITE). Lancet; 349:747-752.

Pitt B, Zannad F, Remme WJ, Cody R, Castaigne A, Perez A, Palensky J, Wittes J (1999): The effect of spironolactone on morbidity and mortality in patients with severe heart failure. Randomized Aldactone Evaluation Study Investigators. N Engl J Med; 341:709-717.

Pitt B, Poole-Wilson PA, Segal R (2000): Effect of losartan compared with captopril on mortality in patients with symptomatic heart failure: randomised trial - the Losartan Heart Failure Survival Study ELITE II. Lancet; 355:1582-1587.

Poole-Wilson PA, Swedberg K, Cleland JG, Di Lenarda A, Hanrath P, Komajda M, Lubsen J, Lutiger B, Metra M, Remme WJ, Torp-Pedersen C, Scherhag A, Skene A; Carvedilol Or Metoprolol European Trial Investigators (2003): Comparison of carvedilol and metoprolol on clinical outcomes in patients with chronic heart failure in the Carvedilol Or Metoprolol European Trial (COMET): randomised controlled trial. Lancet; 362:7-13.

Remme WJ, Riegger G, Hildebrandt P, Komajda M, Jaarsma W, Bobbio M, SolerSoler J, Scherhag A, Lutiger B, Ryden L (2004): The benefits of early combination treatment of carvedilol and an ACE-inhibitor in mild heart failure and left ventricular systolic dysfunction. The Carvedilol and ACE-inhibitor Remodelling Mild Heart Failure Evaluation Trial (CARMEN). Cardiovasc Drugs Ther; 18:57-66.

Roffman DS (2004): High-versus low-dose ACE-Inhibitor therapy in chronic heart failure. Ann Pharmacother; 38:831-838.

Rote Liste Service GmbH: Rote Liste. Arzneimittelverzeichnis für Deutschland. Editio Cantor Verlag für Medizin und Naturwissenschaften $\mathrm{GmbH}$, Aulendorf 2006 
Rutten FH, Grobbee DE, Hoes AW (2003): Differences between general practitioners and cardiologists in diagnosis and management of heart failure: a survey in everyday practice. Eur J Heart Fail; $\underline{5}: 337-344$.

Saudubray T, Saudubray C, Viboud C, Jondeau G, Valleron AJ, Flahault A, Hanslik T (2005): Prevalence and management of heart failure in France: national study among general practitioners of the Sentinelles network. Rev Med Interne; 11:845850.

Swedberg K, Cleland J, Dargie H, Drexler H, Follath F, Komajda M, Tavazzi L,Smiseth OA, Gavazzi A, Haverich A, Hoes A, Jaarsma T, Korewicki J, Levy S, Linde C, Lopez-Sendon JL, Nieminen MS, Pierard L, Remme WJ (2005): Guidelines for the diagnosis and treatment of chronic heart failure: executive summary (update 2005). The Task Force for the Diagnosis and Treatment of Chronic Heart Failure of the European Society of Cardiology. Eur Heart J; 26:1115-1140.

The SOLVD Investigators (1991): Effect of enalapril on survival in patients with reduced left ventricular ejection fractions and congestive heart failure. $\mathrm{N}$ Engl J Med; $\underline{325}: 293-302$.

Tierney WM, Overhage JM, Murray MD, Harris LE, Zhou XH, Eckert GJ, Smith FE, Nienaber N, McDonald CJ, Wolinsky FD (2003): Effects of computerized guidelines for managing heart disease in primary care. J Gen Intern Med; 18:967-976.

Toal M, Walker R (2000): Angiotensin converting enzyme (ACE) inhibitors in the treatment of heart failure in general practice in north Cumbria. Eur $\mathrm{J}$ Heart Fail; 2:201-207.

Van Walraven C, Seth R, Laupacis A (2002): Dissemination of discharge summaries. Not reaching follow-up physicians. Can Fam Physician; 48:737-742.

Varela-Roman A, Grigorian L, Barge E, Bassante P, de la Pena MG, GonzalezJuanatey JR (2005): Heart failure in patients with preserved and deteriorated left ventricular ejection fraction. Heart; 91:489-494. 
Weltgesundheitsorganisation (WHO): Internationale statistische Klassifikation der Krankheiten und verwandter Gesundheitsprobleme. 10. Revision. Version 2005 (2005)

(http://www.dimdi.de/dynamic/de/klassi/diagnosen/icd10/htmlamtl2005/fr-icd.htm, Stand: 1.12 .2005$)$

Wetzel D, Scheidt-Nave C, Rogausch A (2003): Medizinische Versorgung in der Praxis - eine Modellstudie zur Verbesserung der hausärztlichen Versorgungsforschung in Deutschland. Z Allgemeinmed; $\underline{79}$ :394-398.

Wierzchowiecki M, Poprawski K, Nowicka A, Kandziora M, Piatkowska A (2005): What primary care physicians know about diagnosis and treatment of chronic heart failure. Kardiol Pol; $\underline{62: 218-228 . ~}$

Wilhelmsen L, Rosengren A, Eriksson H, Lappas G (2001): Heart failure in the general population of men - morbidity, risk factors and prognosis. J Intern Med; 249:253261. 


\section{$9 \quad$ Anhang}

\section{A.1 Vergleich der Verordnungen mit Daten anderer Studien}

\begin{tabular}{|c|c|c|c|c|}
\hline \multirow[t]{2}{*}{ Stoffgruppen } & \multicolumn{2}{|c|}{ Innerklinische Medikation } & \multicolumn{2}{|c|}{ Hausärztliche Medikation } \\
\hline & $\begin{array}{c}\text { Poststationäre } \\
\text { Gruppe, } \\
\text { Baseline- } \\
\text { Erhebung }\end{array}$ & $\begin{array}{c}\text { Komajda et al. } \\
2003\end{array}$ & $\begin{array}{c}\text { Kontrollgruppe, } \\
\text { Baseline- } \\
\text { Erhebung }\end{array}$ & $\begin{array}{c}\text { Cleland et al. } \\
2002\end{array}$ \\
\hline ACE-Hemmer ${ }^{1}$ & $75,0 \%$ & $79,9 \%$ & $58,0 \%$ & $60,0 \%$ \\
\hline Beta-Blocker $^{2}$ & $83,0 \%$ & $48,9 \%$ & $42,0 \%$ & $34,0 \%$ \\
\hline
\end{tabular}

" Angiotensin-Converting-Enzyme-Inihibitoren (Evidenz A, jeder)

${ }^{2}$ Betaadrenozeptor-Antagonisten (nur Metoprolol, Bisoprolol, Carvedilol)

A.2 Vergleich von empfohlenen Zieldosen und maximalen Tagesdosen der verwendeten Medikamente

\begin{tabular}{lcc}
\hline \hline Wirkstoff & Zieldosis/Tag ${ }^{1}(\mathrm{mg} / \mathrm{d})$ & Maximaldosis/Tag $^{2}(\mathrm{mg} / \mathrm{d})$ \\
\hline Enalapril & 20 & 20 \\
Ramipril & 10 & 10 \\
Benazepril & 10 & 20 \\
Lisinopril & 35 & 35 \\
Captopril & 150 & 150 \\
Metoprolol & 200 & 200 \\
Bisoprolol & 10 & 10 \\
Carvedilol & 50 & 100 \\
\hline \hline
\end{tabular}

${ }^{1}$ empfohlene Zieldosierungen nach Leitlinie der Leitliniengruppe Hessen 2003

${ }^{2}$ Angabe für Maximaldosierungen nach Karow, Lang-Roth 2005 
A.3 Vergleich der durchschnittlichen Arzneimittelkosten für ACEHemmer und Beta-Blocker (aus: Rote Liste Service GmbH, Stand: 27.07.2006)

\begin{tabular}{lccc}
\hline \hline Wirkstoff & $\begin{array}{c}\text { Zieldosis/Tag } \\
(\mathrm{mg} / \mathrm{d})\end{array}$ & \multicolumn{2}{c}{ Apothekenverkaufspreise $^{2}$} \\
& 20 & $38,29 \pm 8,55$ & Günstigstes Präparat $^{4}$ \\
\hline Enalapril & 10 & $51,20 \pm 7,00$ & 29,74 \\
Ramipril & 10 & $37,09 \pm 41,27$ & 44,20 \\
Benazepril & 200 & $32,93 \pm 13,29$ & 32,90 \\
Metoprolol & 10 & $26,43 \pm 1,66$ & 19,64 \\
Bisoprolol & & 24,77 \\
\hline \hline
\end{tabular}

'empfohlene Zieldosierungen nach Leitlinie der Leitliniengruppe Hessen 2003

${ }^{2}$ Apothekenverkaufspreise mit Mehrwertsteuer; Kosten für 100 Tabletten (N3) der Stärke gemäß der jeweiligen empfohlenen Tageszieldosis in $\mathrm{mg}$

${ }^{3}$ alle Werte in Euro, Mittelwert \pm Standardabweichung

${ }^{4}$ alle Werte in Euro 


\section{A.4 Flussdiagramm zur Pharmakotherapie nach klinischen Gesichts- punkten (aus: Leitliniengruppe Hessen: Hausärztliche Leitlinie Chro- nische Herzinsuffizienz 2003, S.14)}

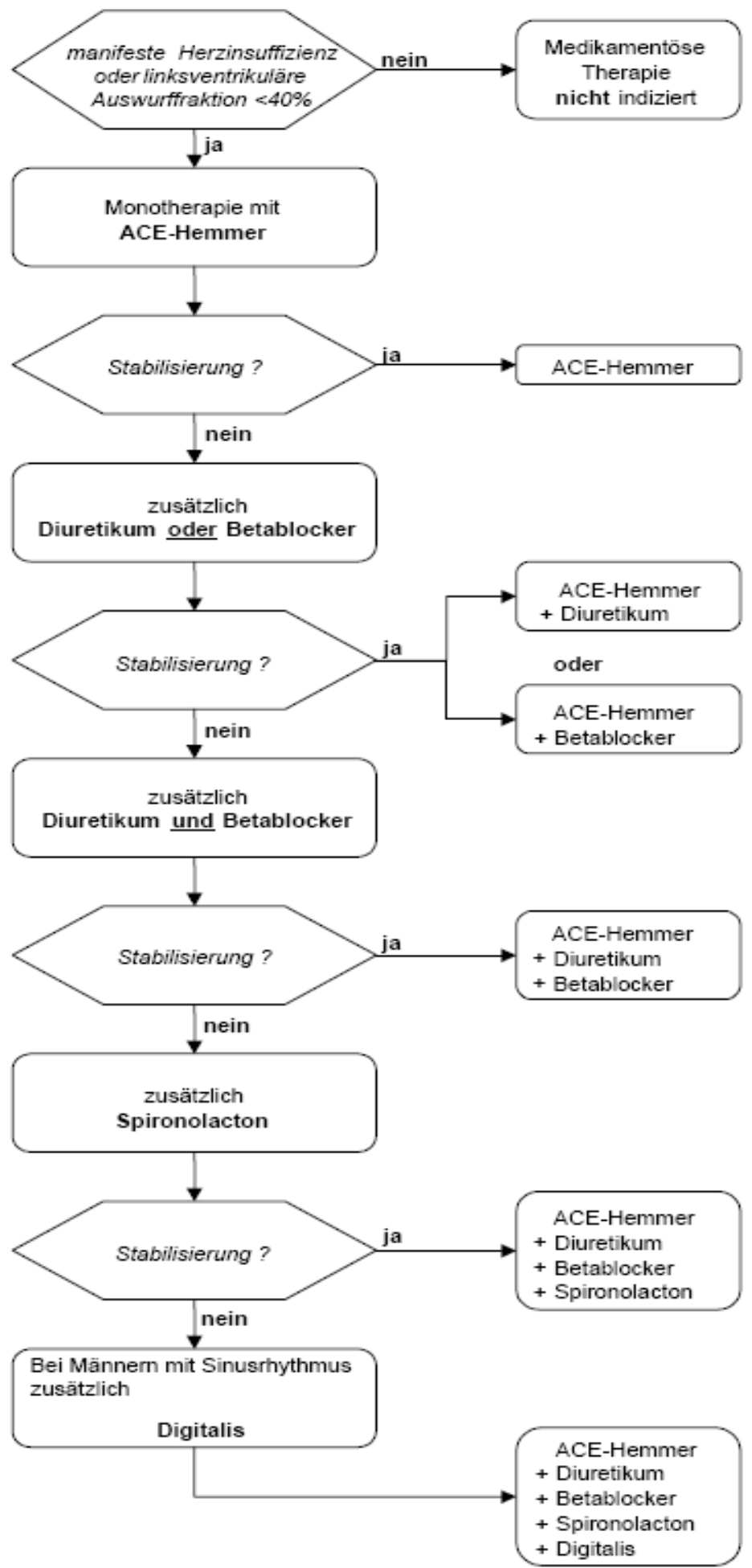




\section{A.5 Aktuelle Empfehlungen zur Therapie der Herzinsuffizienz}

Am 18. Mai 2005 hat die European Society of Cardiology (ESC) die neuste überarbeitete Auflage ihrer Leitlinien zur Behandlung der chronischen Herzinsuffizienz online veröffentlicht (Swedberg et al. 2005). Auch die Leitliniengruppe Hessen hat für September 2005 eine aktualisierte Auflage angekündigt, die jedoch zum gegenwärtigen Zeitpunkt noch aussteht.

Im Wesentlichen haben sich im Vergleich zur letzten Auflage, die unter anderem den Bezug für die Betrachtungen in dieser Arbeit bietet, keine Neuerungen ergeben. Verstärkt wird auf den frühen Einsatz von Beta-Blockern, wie zum Beispiel die Verwendung auch bei asymptomatischen Patienten nach einem kardialen Ereignis, ganz nach amerikanischem Vorbild, gedrängt. Hier kommen Beta-Blocker bereits im AHAStadium B (entsprechend NYHA I), also beim asymptomatischen Patienten, zum Einsatz (Tabelle 7, S.26). Für den frühzeitigen kombinierten Einsatz von ACEHemmern und Beta-Blockern sprachen sich auch Remme et al. (2004) aus. Eine Untersuchung an 572 Patienten mit geringgradiger Herzinsuffizienz zeigte für die Kombinationstherapie von Enalapril und Carvedilol einen signifikant stärkeren Rückgang von systolischer Dysfunktion und Remodelling nach 18 Monaten im Vergleich zu den Gruppen mit Monatherapie (nur Enalapril, nur Carvedilol).

Im Bereich der sicher prognoseverbessernden Medikamente (Evidenz A) hat jetzt Nebivolol, ein weiterer Beta-Blocker, eine Zulassung für die Behandlung der chronischen Herzinsuffizienz erlangt. In einer placebokontrollierten Doppelblindstudie konnten Flather et al. (2005) an 2128 Patienten mit chronischer Herzinsuffizienz eine signifikant geringere Gesamtmortalität und eine niedrigere Rehospitalisierungsrate in der Nebivolol-Gruppe zeigen. Die aus dieser Studie abgeleitete Zieldosis wird in den neuen ESC-Leitlinien mit $10 \mathrm{mg} / \mathrm{Tag}$ angegeben. Für den Hausarzt könnte dies insofern bedeutsam sein, als dass nun eine Substanz als wirksam befunden wurde, die ohnehin schon lange verschrieben wird.

Mit Blick auf die Problematik des umstrittenen Nutzens der Hochdosistherapie muss gesagt werden, dass diese Diskussion bisher keinen Eingang in die Empfehlungen der Leitlinien genommen hat. Die angegebenen Zieldosen entsprechen denen der letzten Ausgabe mit Verweis auf die bekannten Studien (Abschnitt 5.5, S.41). 


\section{A.6 Patientenaufklärung}

\section{Patientenaufklärung zur Studie}

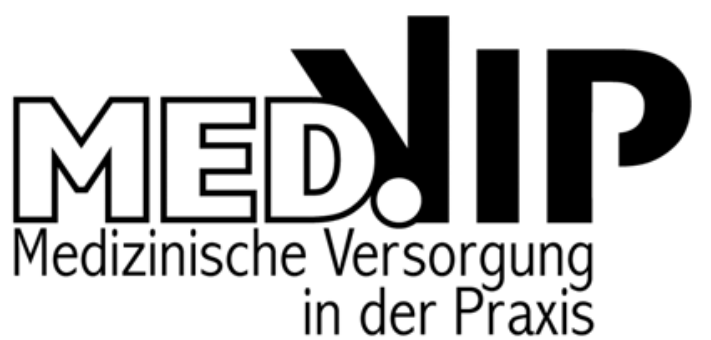

Sehr geehrte Patientin, sehr geehrter Patient,

Sie sind hier im Universitätsklinikum stationär aufgenommen worden, weil Sie unter anderem an einer Herzerkrankung leiden. Bevor Sie nun nach Hause gehen, bitten wir Sie um Ihre Mitwirkung bei einer Studie, die der Verbesserung der Versorgung von Patienten dienen soll, die an einer Herzinsuffizienz („Herzschwäche“, Schwäche des Herzmuskels) leiden. Hierzu möchten wir persönlich und telefonisch mit Ihnen in Kontakt treten, um den Schweregrad Ihrer Erkrankung, Ihr Befinden und Ihre aktuelle Behandlung zu erfragen.

Wir erhoffen uns von dieser Untersuchung wertvolle Hinweise für eine noch bessere Behandlung der Herzschwäche in Klinik und Praxis. Daher bitten wir um Ihr Einverständnis, Sie in unsere Studie aufnehmen zu dürfen.

Eine Änderung Ihrer Behandlung ist nicht vorgesehen, aus der Teilnahme erwächst Ihnen kein gesundheitliches Risiko. Es handelt sich lediglich um eine Befragung, Sie müssen nach Ihrer Entlassung nicht zur Beantwortung der Fragen ins Universitätsklinikum kommen, dies geschieht per Post (mit einem Fragebogen) oder telefonisch.

Es soll zunächst (nach Vorliegen Ihrer Einverständniserklärung) eine Befragung vor der Entlassung durchgeführt werden. Etwa 7 Tage nach Ihrer Entlassung würden wir Sie zuhause anrufen und Ihnen einige kurze Fragen zur aktuellen Behandlung stellen. Anschließend werden im Abstand von sechs Monaten insgesamt drei Befragungen durchgeführt, um den Verlauf Ihrer Erkrankung zu erfassen. Danach wird ein kurzes Gespräch mit Ihrem Hausarzt über Ihre Erkrankung und deren Behandlung geführt werden, außerdem möchten wir die Behandlungsdaten, die bei Ihrem Hausarzt über Sie vorliegen, auswerten.

Ihre Angaben unterliegen dem Datenschutz. Alle Mitarbeiter sind an die Schweigepflicht gebunden, eine Weitergabe Ihrer Daten an Dritte erfolgt unter keinen Umständen.

Selbstverständlich ist die Teilnahme an der Studie absolut freiwillig, Sie haben auch während der Studie jederzeit die Möglichkeit, ohne Angabe von Gründen Ihre Einwilligung zu widerrufen, ohne dass Ihnen ein Nachteil entsteht.

Sollten Sie Fragen haben, wenden Sie sich bitte an

- Dr. Dirk Wetzel, Facharzt für Allgemeinmedizin, Abteilung Allgemeinmedizin der Georg-August-Universität, Humboldtallee 38, 37073 Göttingen, Tel. 0551/39-14227 
Behandlung der Herzschwäche in Klinik und Praxis

(Pharmakotherapie der Herzinsuffizienz

an der ambulant-stationären Schnittstelle

-poststationäre Verlaufsbeobachtung-)

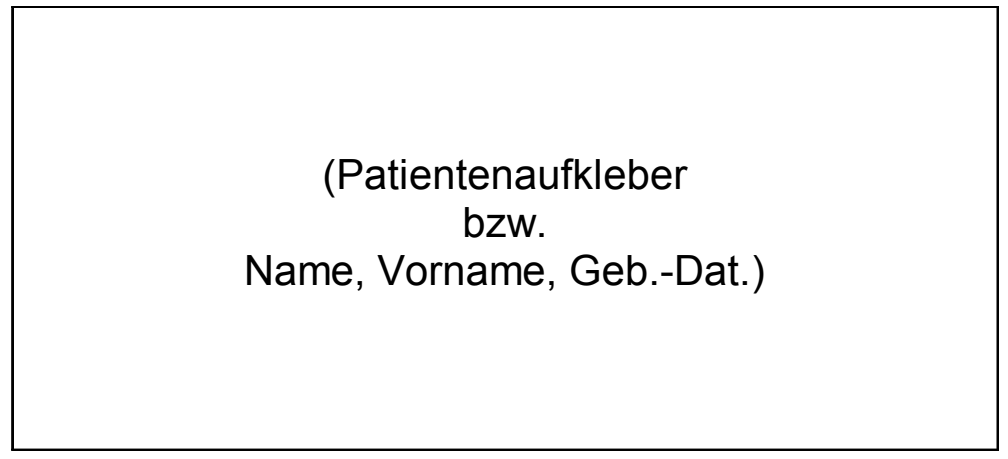

Hiermit erkläre ich mein Einverständnis, an der oben genannten Studie teilzunehmen. Ich wurde über den Inhalt der Studie und die geplanten Maßnahmen informiert und hatte Gelegenheit, den mir ausgehändigten Aufklärungsbogen sorgfältig zu lesen. Alle meine Fragen im Zusammenhang mit der Studie wurden hinreichend beantwortet. Der Speicherung meiner Daten in anonymisierter Form stimme ich zu.

Ich bin damit einverstanden, dass das behandelnde Krankenhaus und mein Hausarzt im Rahmen dieser Studie Auskunft über meine Erkrankungen und deren Behandlungen geben dürfen, eine Kopie des Arztbriefes darf an die Abteilung Allgemeinmedizin gesandt werden.

Diese Entbindung von der Schweigepflicht gilt nur für diese Studie. Meine Angaben werden streng vertraulich behandelt und keinesfalls an Dritte weitergegeben.

Mir ist bekannt, dass ich meine Einwilligung jederzeit ohne Angabe von Gründen widerrufen kann, ohne dass mir hieraus irgendwelche Nachteile entstehen.

, den

Patientennummer

(Unterschrift des Patienten)

Station

(Unterschrift der aufklärenden Person)

Ich möchte nicht an der Studie teilnehmen, bin aber mit der anonymen Erfassung meiner Initialen, meines Geburtsdatums und der Schwere meiner Erkrankung anhand der Patientenakte einverstanden. den 


\section{A.7 Fragebogen Baseline-Erhebung}

1. Patientencode: Station:

Hz.:

2. Geschlecht $\square$ männlich $\square$ weiblich

3. Geburtsjahr

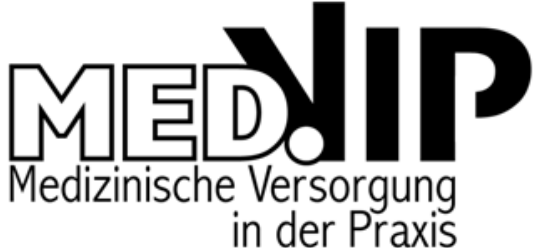

4. Name/Adresse des Hausarztes:

\section{FRAGEN AN DEN PATIENTEN:}

NYHA IV Leiden Sie in Ruhe unter Luftnot?

NYHA III Haben Sie Luftnot bei leichter Belastung: Gang zur Toilette/Gehen in der Ebene?

NYHA II Haben Sie Luftnot bei stärkerer Belastung: Treppensteigen/Bergauflaufen?

NYHA I

\section{NYHA-Klassifikation \\ $\square$ I $\quad \square$ II $\quad \square$ III $\quad \square$ IV}

6. Wissen Sie an welcher Erkrankung/welchen Erkrankungen Sie leiden? ja $\square \quad$ nein $\square$ Wenn ja, welche?

7. Welcher Arzt betreut Sie ambulant mit Ihrer Herzerkrankung vorwiegend?

(Name, Anschrift, Fachrichtung - fehlendes ggf. aus Ärzteverzeichnis o. Tel.-Buch ergänzen)

8. Wie regelmäßig sind Sie bei diesem Arzt in Betreuung?

regelmäßige, feste Termine (z.B. alle 2 oder 4 Wochen) unregelmäßige Termine nur bei Bedarf (Termine nur auf Initiative des Pat.)

9. Ernähren Sie sich kochsalzarm?

ja $\square \quad$ nein $\square$

10. Kontrollieren Sie Ihre Trinkmenge?

Wenn ja, wie viel trinken Sie etwa täglich?

ja $\square \quad$ nein $\square$

11. Wie häufig wiegen Sie sich? nie/seltener als 1 mal pro Monat $\square$

11. Wie häufig wiegen Sie sich? nie/seltener als 1 mal pro Monat $\square$

Liter

ca. 1 mal pro Monat ca. 1 mal pro Woche mehrmals pro Woche/täglich

12. Wenn Sie innerhalb von zwei Tagen 2-3 Kilo zunehmen würden, was würden Sie tun? (Nennt der Patient:

a) „Arzt kontaktieren/aufsuchen“ ja $\square \quad$ nein $\square$ b) „Wassertabletten/Diuretika erhöhen“ ja $\square \quad$ nein $\square$ )

13. Lassen Sie sich jährlich gegen Grippe (Influenza) impfen? ja $\square \quad$ nein

14. Lassen Sie sich regelmäßig gegen Pneumokokken (Lungenentzündung) impfen? ja $\square \quad$ nein $\square$

15. Leiden Sie unter einer Unverträglichkeit oder Allergie gegenüber einem bestimmten Medikament? Wenn ja, welche?

ja $\square \quad$ nein $\square$

16. Dürfen Sie aus anderen Gründen bestimmte Medikamente nicht nehmen?

ja $\square \quad$ nein $\square$ Wenn ja, welche? 
17. Welche Herz-Medikamente haben Sie vor dem Aufenthalt eingenommen?

Bitte geben Sie auch die Dosis an.

weiß nicht $\square$

Medikamente/Dosis:

\begin{tabular}{|l|l|l|l|l|}
\hline Medikament & morgens & mittags & abends & zur Nacht \\
\hline & & & & \\
\hline & & & & \\
\hline & & & & \\
\hline & & & & \\
\hline & & & & \\
\hline & & & & \\
\hline & & & & \\
\hline & & & & \\
\hline
\end{tabular}

sind diese hier geändert worden?

ja $\square \quad$ nein $\square$

weiß nicht $\square$

18. Wurde mit Ihnen über die Änderung gesprochen? $\quad$ ja $\square \quad$ nein $\square$

\section{EINWEISUNGSSCHEIN/VERLEGUNGSBRIEF/KLINIKAKTE}

19. Einweisung über

$$
\begin{aligned}
& \square \text { Hausarzt } \\
& \square \text { ärztlichen Notdienst } \\
& \square \text { privat gekommen }
\end{aligned}
$$

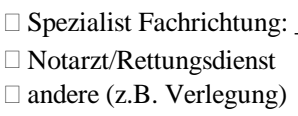

20. Einweisungsdiagnose(n):

(lt. Einweisungsschein/Verl.-Brief)

21. Wurde die Herzinsuffizienz bei Einweisung aufgeführt? $\quad$ ja $\square \quad$ nein $\square$

22. Symptome der Herzinsuffizienz:

(bei Einweisung/lt. Aufnahmebefund)

23. Kontraindikationen/Unverträglichkeiten gegen:

(lt. Einweisungsschein/Verl.-Brief)

24. Medikation bei Einweisung:

-Vormedikation-

(mögl. genauer Präparatename, Dosis und generischer Name)

\begin{tabular}{|l|l|l|l|l|}
\hline Medikament & morgens & mittags & abends & zur Nacht \\
\hline & & & & \\
\hline & & & & \\
\hline & & & & \\
\hline & & & & \\
\hline & & & & \\
\hline & & & & \\
\hline & & & & \\
\hline & & & & \\
\hline
\end{tabular}


AKTE

25. NYHA-Klassifikation

$\square$ I $\quad \square$ II $\quad \square$ III $\square$ IV $\square$ nicht dokumentiert (lt. Aufnahmebefund)

26. In der Klinik durchgeführte kardiologische Diagnostik: $\square$ Ergometrie (lt. Akte)

$\square$ Herzkatheter

$\square$ andere:

27. Echokardiografie (bei mehreren den letzten Befund) $\quad \square$ ja $\quad \square$ nein

Wenn ja, Kurzbefund:

28. Ejektionsfraktion: $\%$

29. Aktuelle Medikation bei Erhebung (Med. aus der Planette) :

\begin{tabular}{|l|l|l|l|l|}
\hline Medikament & morgens & mittags & abends & zur Nacht \\
\hline & & & & \\
\hline & & & & \\
\hline & & & & \\
\hline & & & & \\
\hline & & & & \\
\hline & & & & \\
\hline & & & & \\
\hline & & & & \\
\hline & & & & \\
\hline
\end{tabular}




\section{A.8 Telefoninterview (exemplarisch aus der Follow-Up-Untersuchung)}

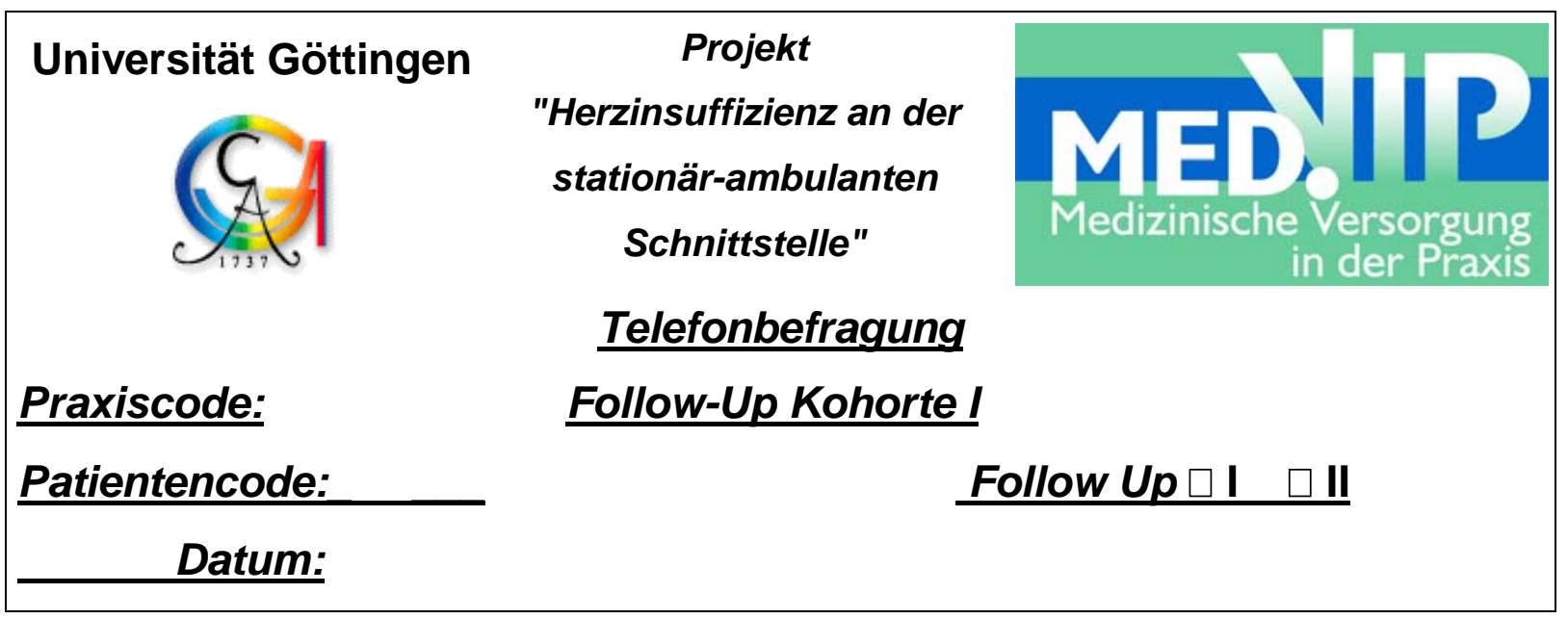

\section{Guten Tag Frau ... \\ Guten Tag Herr ...}

...hier ist...(Name der Study Nurse) von der Abteilung Allgemeinmedizin der Universität Göttingen.

Es geht um die Befragung zur Herzschwäche, an der Sie teilnehmen. Wir hatten Ihnen schon einmal vor einigen Monaten einen Fragebogen zugeschickt. Ich würde Ihnen nun nochmals gerne einige Fragen stellen - haben Sie dafür jetzt Zeit? Die Befragung wird ungefähr 10-15 Minuten dauern.

(wenn jetzt Zeit - weiter !)

(wenn zur Zeit ungünstig - möglichst Termin für Gespräch vereinbaren)

Haben Sie jetzt noch Fragen, bevor ich mit dem Interview beginne?...

Gut, dann fange ich jetzt an...

Fragen zur Behandlung der Herzschwäche

Nun folgen einige Fragen zu der Behandlung Ihrer Herzschwäche.

Versuchen Sie sich bitte an die Behandlungen zu erinnern, die Sie

innerhalb der letzten 9 Monate erhalten haben (seit letzter Befragung am....). Dabei geht es nur um Arztbesuche, Untersuchungen und Behandlungen, die Sie wegen Ihrer

Herzkrankheit erhalten haben. 
- persönliche Arztkontakte

1) Welche Ärzte haben Sie in den letzten 9 Monaten wegen Ihrer Herzerkrankung aufgesucht? Ich lese Ihnen einige vor. Falls Sie dort waren, versuchen Sie bitte abzuschätzen, wie oft sie bei dem jeweiligen Arzt waren.

\begin{tabular}{|c|c|c|c|}
\hline $\begin{array}{l}\text { Waren Sie in den letzten } 9 \\
\text { Monaten beim... }\end{array}$ & & & $\begin{array}{l}\text { Wie oft waren Sie dort? } \\
\text { (bitte eintragen) }\end{array}$ \\
\hline Hausarzt/hausärztl. Internist & $\mathrm{ja} \square$ & nein $\square$ & Anzahl Besuche: \\
\hline Kardiologe & $\mathrm{ja} \square$ & nein $\square$ & Anzahl Besuche: \\
\hline Radiologe (Röntgenarzt) & $\mathrm{ja} \square$ & nein $\square$ & Anzahl Besuche: \\
\hline Psychotherapeut & $\mathrm{ja} \square$ & nein $\square$ & Anzahl Besuche: \\
\hline andere Ärzte & $\mathrm{ja} \square$ & nein $\square$ & $\begin{array}{l}\text { Anzahl Besuche: } \\
\text { Welche Ärzte: }\end{array}$ \\
\hline
\end{tabular}

2) Welcher Arzt betreut Sie wg. Ihrer Herzschwäche hauptsächlich?

$\square$ Hausarzt (auch hausärztl. Internist)

$\square$ Spezialist (Kardiologe)

$\square$ anderer Arzt welcher?

3) Wurden Sie innerhalb der letzten neun Monate wegen Ihrer Herzerkrankung ins Krankenhaus eingewiesen?

ja $\square$ nein $\square$ (weiter zur nächsten Frage!)

In welches Krankenhaus/welche Krankenhäuser? 
- persönliche Arztkontakte

1) Welche Ärzte haben Sie in den letzten 9 Monaten wegen Ihrer Herzerkrankung aufgesucht? Ich lese Ihnen einige vor. Falls Sie dort waren, versuchen Sie bitte abzuschätzen, wie oft sie bei dem jeweiligen Arzt waren.

\begin{tabular}{|c|c|c|c|}
\hline $\begin{array}{l}\text { Waren Sie in den letzten } 9 \\
\text { Monaten beim... }\end{array}$ & & & $\begin{array}{l}\text { Wie oft waren Sie dort? } \\
\text { (bitte eintragen) }\end{array}$ \\
\hline Hausarzt/hausärztl. Internist & $\mathrm{ja} \square$ & nein $\square$ & Anzahl Besuche: \\
\hline Kardiologe & $\mathrm{ja} \square$ & nein $\square$ & Anzahl Besuche: \\
\hline Radiologe (Röntgenarzt) & $\mathrm{ja} \square$ & nein $\square$ & Anzahl Besuche: \\
\hline Psychotherapeut & $\mathrm{ja} \square$ & nein $\square$ & Anzahl Besuche: \\
\hline andere Ärzte & $\mathrm{ja} \square$ & nein $\square$ & $\begin{array}{l}\text { Anzahl Besuche: } \\
\text { Welche Ärzte: }\end{array}$ \\
\hline
\end{tabular}

2) Welcher Arzt betreut Sie wg. Ihrer Herzschwäche hauptsächlich?

$\square$ Hausarzt (auch hausärztl. Internist)

$\checkmark$ Spezialist (Kardiologe)

$\square$ anderer Arzt welcher?

3) Wurden Sie innerhalb der letzten neun Monate wegen Ihrer Herzerkrankung ins Krankenhaus eingewiesen?

ja $\square$ nein $\square$ (weiter zur nächsten Frage!)

In welches Krankenhaus/welche Krankenhäuser? 


\begin{tabular}{|c|c|}
\hline $\begin{array}{l}\text { Wie häufig waren Sie im } \\
\text { Krankenhaus? }\end{array}$ & $\begin{array}{l}\text { Welche Therapie haben Sie im } \\
\text { Krankenhausbekommen?: }\end{array}$ \\
\hline -mal & $\square$ nur Medikamente \\
\hline \multirow{6}{*}{$\begin{array}{l}\text { Wie viele Tage waren } \\
\text { Sie insgesamt im } \\
\text { Krankenhaus? }\end{array}$} & $\begin{array}{l}\square \text { Herzkatheter mit Aufdehnung von } \\
\text { Gefäßen (PTCA) }\end{array}$ \\
\hline & $\square$ Bypass-Operation (ACVB) \\
\hline & $\begin{array}{l}\square \text { Einpflanzung eines } \\
\text { Herzschrittmachers }\end{array}$ \\
\hline & $\square$ Einpflanzung eines Defibrillators \\
\hline & $\square$ Einpflanzung eines Kunstherzens \\
\hline & $\begin{array}{l}\square \text { Herztransplantation } \\
\text { Andere: }\end{array}$ \\
\hline
\end{tabular}

4) Ich lese Ihnen jetzt vor, welche Medikamente Sie nach unseren Unterlagen vor 9 Monaten eingenommen haben. Gibt es darunter Medikamente, die Sie jetzt NICHT MEHR einnehmen?( Medikamente It. Baseline bzw. letztem Follow-Up vorlesen)

ja $\square$ nein $\square$ falls nein: bitte weiter bei Frage 3!

\begin{tabular}{|l|l|l|l|l|}
\hline Medikament & morgens & mittags & abends & zur Nacht \\
\hline & & & & \\
\hline & & & & \\
\hline & & & & \\
\hline & & & & \\
\hline & & & & \\
\hline & & & & \\
\hline & & & & \\
\hline & & & & \\
\hline
\end{tabular}

\begin{tabular}{|c|c|c|}
\hline $\begin{array}{l}\text { Wie ist der Name des } \\
\text { Medikaments? }\end{array}$ & $\begin{array}{l}\text { Wann wurde es } \\
\text { abgesetzt? }\end{array}$ & Warum? \\
\hline \multirow[t]{3}{*}{ 1) } & & $\square$ nicht mehr vertragen \\
\hline & & $\begin{array}{l}\square \text { nicht mehr ausreichend gewirkt (neues } \\
\text { „stärkeres“ Medikament bekommen) }\end{array}$ \\
\hline & & $\square$ andere Gründe: \\
\hline \multirow[t]{3}{*}{ 2) } & & $\square$ nicht mehr vertragen \\
\hline & & $\begin{array}{l}\square \text { nicht mehr ausreichend gewirkt (neues } \\
\text { „stärkeres“ Medikament bekommen) }\end{array}$ \\
\hline & & $\square$ andere Gründe: \\
\hline \multirow[t]{3}{*}{ 3) } & & $\square$ nicht mehr vertragen \\
\hline & & $\begin{array}{l}\square \text { nicht mehr ausreichend gewirkt (neues } \\
\text { „stärkeres“ Medikament bekommen) }\end{array}$ \\
\hline & & $\square$ andere Gründe: \\
\hline
\end{tabular}


5) Nehmen Sie derzeit Medikamente ein, die nicht in der von mir vorgelesenen Liste enthalten waren, also Medikamente, die im letzten halben Jahr NEU verordnet wurden?

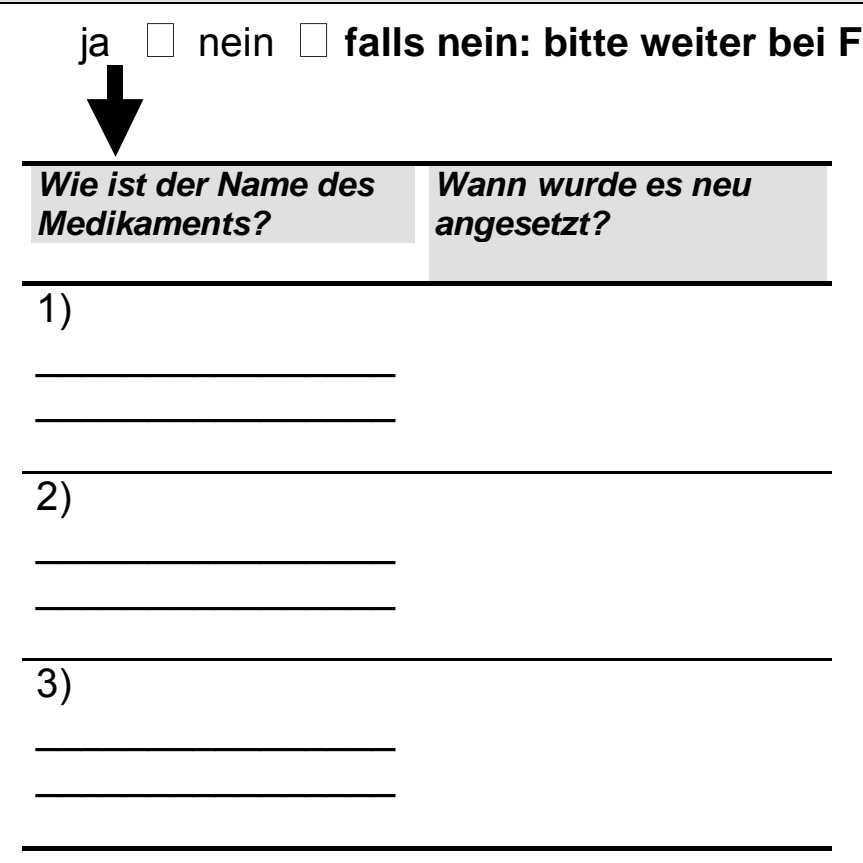

6) Um ganz sicher zu gehen, dass ich die von Ihnen derzeit eingenommenen Medikamente richtig notiert habe, lese ich Ihnen jetzt alle Medikamente, die Sie nach unseren Unterlagen derzeit einnehmen, vor....... Ist diese Aufzählung korrekt?

ja $\square$ (weiter mit nächster Frage) nein

Medikamentenliste:

korrekte

\begin{tabular}{|l|l|l|l|l|}
\hline Medikament & morgens & mittags & abends & zur Nacht \\
\hline & & & & \\
\hline & & & & \\
\hline & & & & \\
\hline & & & & \\
\hline & & & & \\
\hline & & & & \\
\hline & & & & \\
\hline & & & & \\
\hline
\end{tabular}


7) Wurden bei Ihrem Hausarzt oder beim Spezialisten noch weitere Untersuchungen durchgeführt?

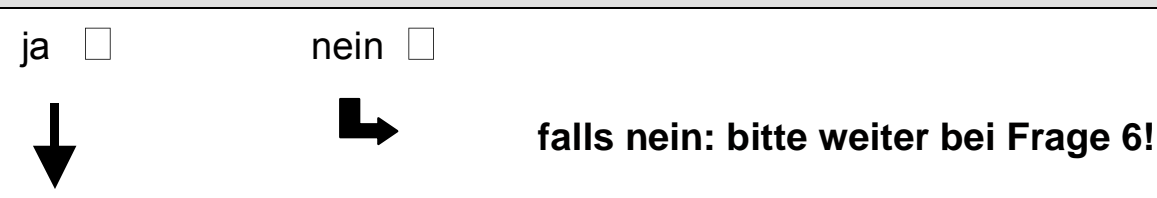

\begin{tabular}{|c|c|c|}
\hline $\begin{array}{l}\text { Welche Art der Untersuchung wurde bei Ihnen } \\
\text { durchgeführt? } \\
\text { Ich lese Ihnen einige vor. Sagen Sie mir bitte immer, ob } \\
\text { dies gemacht wurde und wie oft es in den letzten } 9 \\
\text { Monaten getan wurde }\end{array}$ & & $\begin{array}{l}\text { Wie oft wurde } \\
\text { dies in den } \\
\text { letzten } 9 \\
\text { Monaten } \\
\text { gemacht? } \\
\text { (bitte eintragen) } \\
\end{array}$ \\
\hline Elektrokardiogramm (EKG) & $\begin{array}{l}\square \text { ja } \rightarrow \rightarrow \\
\square \text { nein }\end{array}$ & $\begin{array}{r}\text { Anzahl } \\
\text { mal }\end{array}$ \\
\hline Belastungs-EKG (Fahrrad + EKG) & $\begin{array}{l}\square \text { ja } \rightarrow \rightarrow \\
\square \text { nein }\end{array}$ & $\begin{array}{r}\text { Anzahl } \\
-\quad \mathrm{mal}\end{array}$ \\
\hline Echokardiographie (Herzultraschall) & $\begin{array}{l}\square \text { ja } \rightarrow \rightarrow \\
\square \text { nein }\end{array}$ & $\begin{array}{c}\text { Anzahl } \\
\text { mal } \\
\end{array}$ \\
\hline $\begin{array}{l}\text { Stressechokardiographie (Herzultraschall nach einer } \\
\text { Spritze in den Arm) }\end{array}$ & $\begin{array}{l}\square \text { ja } \rightarrow \rightarrow \\
\square \text { nein }\end{array}$ & $\begin{array}{r}\text { Anzahl } \\
\quad \text { mal } \\
\end{array}$ \\
\hline Herzkatheter & $\begin{array}{l}\square \text { ja } \rightarrow \rightarrow \\
\square \text { nein }\end{array}$ & $\begin{array}{c}\text { Anzahl } \\
\quad \text { mal } \\
\end{array}$ \\
\hline $\begin{array}{l}\text { Myokardszintigraphie (Aufnahmen in der } \\
\text { Nuklearmedizin nach einer Spritze in den Arm) }\end{array}$ & $\begin{array}{l}\square \text { ja } \rightarrow \rightarrow \\
\square \text { nein }\end{array}$ & $\begin{array}{r}\text { Anzahl } \\
-\quad \text { mal } \\
\end{array}$ \\
\hline Langzeit-EKG & $\begin{array}{l}\square \text { ja } \rightarrow \rightarrow \\
\square \text { nein }\end{array}$ & $\begin{array}{c}\text { Anzahl } \\
\quad \text { mal } \\
\end{array}$ \\
\hline $\begin{array}{l}\text { Wurde eine andere Untersuchung gemacht? } \\
\text { (welche?-bitte hier eintragen) }\end{array}$ & $\begin{array}{l}\square \text { ja } \rightarrow \rightarrow \\
\square \text { nein }\end{array}$ & $\overline{\mathrm{mal}}$ \\
\hline
\end{tabular}

8) Wurden Sie innerhalb der letzten 9 Monaten wegen Ihrer Herzschwäche krankgeschrieben?

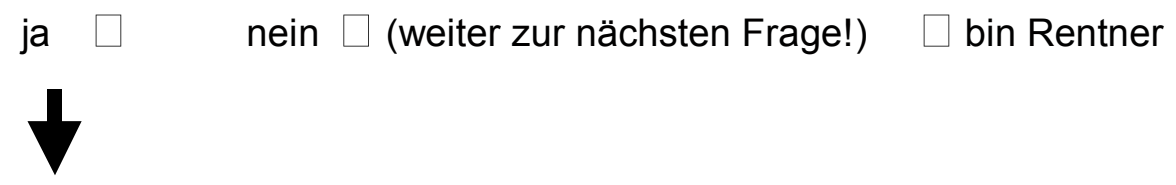

Für wie viele Tage wurden Sie krankgeschrieben?: 
9) Haben Sie in den letzten 9 Monaten noch weitere Behandlungen wegen Ihrer Herzerkrankung erhalten?

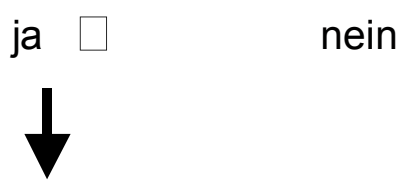

Welche?:

Ich stelle Ihnen nun noch einige Fragen zu Ihrer Erkrankung und zur allgemeinen Lebensführung.

10) Welche Symptome und körperliche Beschwerden Ihrer Herzschwäche/Herzinsuffizienz kennen Sie?

(nicht vorlesen, nur ankreuzen, wenn Pat. es nennt)

$\square$ Atemnot

dicke Beine/Wasseransammlungen/Schwellung/Ödeme

Müdigkeit/Leistungsschwäche

andere:

weiß nicht

11) Kennen Sie Maßnahmen der allgemeinen Lebensführung (also z.B. Ernährung, Vorsichtsmassnahmen etc.), die bei Ihrer Herzschwäche wichtig sind? Welche kennen Sie?

Wichtig sind:

(nicht vorlesen!, nur ankreuzen, wenn Pat. es nennt)

$\square$ salzarme Ernährung

Beschränkung der Trinkmenge

Häufiges Wiegen

Nicht Rauchen

Wenig/Keinen Alkohol trinken

Regelmäßiges, leichtes körperliches Training

Regelmäßige Medikamenteinnahme

Regelmäßig zum Arzt gehen

Bei Verschlechterung zum Arzt gehen/Arzt kontaktieren

Sonstiges: 
11a) Was tun Sie selbst, um einer Verschlechterung Ihrer Herzerkrankung vorzubeugen?

11b) Bei welchen Anzeichen der Verschlechterung würden Sie einen Arzt aufsuchen?

12) Wenn Sie Informationen über Ihre Krankheit brauchen, wie wichtig sind die folgenden Informationsquellen für Sie? (erst alle vorlesen, dann einzeln mit Einteilung 1=unwichtige Informationsquelle bis $5=$ sehr wichtige Informationsquelle)

Fernsehen

Zeitschriften/Zeitungen

Broschüren/Informationsschriften

Internet

Hausarzt/Hausärztin

Herzspezialist (Internist/Kardiologe)

Apotheker/-in

Krankenschwester/-pfleger

Arzthelferin

Ernährungsberater/-in

Freunde/Familie unwichtig neutral sehr wichtig

$\square 1 \quad \square 2 \quad \square 3 \quad \square 4 \quad \square 5$

$\square 1 \quad \square 2 \quad \square 3 \quad \square 4 \quad \square 5$

$\square 1 \quad \square 2 \quad \square 3 \quad \square 4 \quad \square 5$

$\square 1 \quad \square 2 \quad \square 3 \quad \square 4 \quad \square 5$

$\square 1 \quad \square 2 \quad \square 3 \quad \square 4 \quad \square 5$

$\square 1 \quad \square 2 \quad \square 3 \quad \square 4 \quad \square 5$

$\square 1 \quad \square 2 \quad \square 3 \quad \square 4 \quad \square 5$

$\square 1 \quad \square 2 \quad \square 3 \quad \square 4 \quad \square 5$

$\square 1 \quad \square 2 \quad \square 3 \quad \square 4 \quad \square 5$

$\square 1 \quad \square 2 \quad \square 3 \quad \square 4 \quad \square 5$

$\square 1 \quad \square 2 \quad \square 3 \quad \square 4 \quad \square 5$ 
13) a) Hat ein Arzt schon einmal mit Ihnen über kochsalzarme Ernährung gesprochen? Facharzt

Wenn ja, welcher Arzt? $\square$ Hausarzt

Klinikarzt $\quad \square$ niedergelassener

b) Ernähren Sie sich kochsalzarm?

ja $\square \quad$ nein

c) wenn ja, wie viel Kochsalz verwenden Sie? (Mehrfachantworten möglich)

sowenig wie möglich

ich salze nicht zusätzlich nach

weniger als

Gramm

weiß nicht

d) Weshalb könnte kochsalzarme Ernährung bei Ihrer Herzschwäche wichtig sein?

(nicht vorlesen, nur ankreuzen, wenn Pat. es nennt)

Symptome der Herschwäche werden sonst schlimmer

Wassereinlagerung/Ödeme/dicke Beine/Schwellung

weiß nicht

andere Antworten:

14) a) Hat ein Arzt schon einmal mit Ihnen über die Beschränkung der täglichen Trinkmenge gesprochen?

Facharzt

$\begin{array}{rlr}\text { Wa } \square \quad & \text { nein } \square \\ \text { Wenn ja, welcher Arzt? } \square \text { Hausarzt } \quad \square \text { Klinikarzt } \quad \square \text { niedergelassener }\end{array}$

b) Beschränken Sie Ihre Trinkmenge?

Wenn ja, wie viel trinken Sie etwa täglich?

ja $\square \quad$ nein $\square$ Liter

c) Weshalb könnte die Beschränkung Ihrer Trinkmenge bei Ihrer Erkrankung wichtig sein?

(nicht vorlesen, nur ankreuzen, wenn Pat es sagt)

$\square$ Symptome der Herschwäche werden sonst schlimmer

$\square$ Wassereinlagerung/Ödeme/dicke Beine/Schwellung

$\square$ weiß nicht

andere Antworten:

15) a) Hat ein Arzt schon einmal mit Ihnen über häufige Bestimmung Ihres

Körpergewichts (Wiegen) gesprochen? ja $\square \quad$ nein $\square$

Facharzt

Wenn ja, welcher Arzt? $\square$ Hausarzt $\quad \square$ Klinikarzt $\square$ niedergelassener

b) Wie häufig wiegen Sie sich? nie/seltener als 1 mal pro Monat

ca. 1 mal pro Monat

ca. 1 mal pro Woche

mehrmals pro Woche/täglich 
Kontrolle der Symptome der Herschwäche

Kontrolle von Wassereinlagerung/Ödeme/dicke Beine/Schwellung

weiß nicht

andere Antworten:

16) Wenn Sie innerhalb von zwei Tagen 2-3 Kilo zunehmen würden, was würden Sie tun?

$\begin{array}{rlll}\text { (Nennt der Patient: a) } \quad \text { "Arzt kontaktieren/aufsuchen“ } & \text { ja } \square \quad \text { nein } \square \\ \text { b) } & \text { "Wassertabletten/Diuretika erhöhen“ } & \text { ja } & \text { nein }\end{array}$

17. Rauchen Sie?

ja $\square \quad$ nein

Wenn ja: Hat ein Arzt mit Ihnen schon einmal darüber gesprochen, dass Sie das Rauchen aufgeben sollten?

ja $\square$ nein $\square$

Wenn ja, welcher Arzt?: $\square$ Hausarzt $\quad \square$ Klinikarzt $\square$ niedergelassener Facharzt

18.

a) Haben Sie sich im letzten Jahr gegen Grippe impfen lassen? $\quad$ ja $\square \quad$ nein

b) Sind Sie schon einmal gegen Pneumokokken (Lungenentzündung) geimpft worden?

ja $\square \quad$ nein

Vielen Dank für das Gespräch. Wir senden Ihnen nun noch einen Fragebogen zu Ihrem seelischen Befinden zu. Senden Sie uns bitte diesen Bogen ausgefüllt zurück. 


\section{A.9 Fragebogen (Auszug aus der Follow-Up-Untersuchung)}

\section{Fragebogen zu Ihrer derzeitigen Belastbarkeit und Ihren Beschwerden}

Mit der ersten Frage soll eingeschätzt werden, ob und wie stark Sie derzeit unter Luftnot leiden.

FRAGE 1

Leiden Sie derzeit unter LUFTNOT?

$\square_{0}$ NEIN, überhaupt nicht $\rightarrow$ weiter mit FRAGE 2

JA, ich leide unter Luftnot und zwar

$\square_{1}$ in Ruhe $\rightarrow$ weiter mit FRAGE 2

$\square_{2}$ bei geringster körperlicher Belastung (z.B. Gang zur Toilette) $\rightarrow$ weiter mit FRAGE 2

$\square_{3}$ bei leichter körperlicher Belastung (z.B. eine Etage Treppensteigen) $\rightarrow$ weiter mit FRAGE 2

$\square_{4}$ bei starker körperlicher Belastung (z.B. rasches Bergaufgehen) $\rightarrow$ weiter mit FRAGE 2

Mit den folgenden Fragen möchten wir abschätzen, wie stark Sie sich derzeit (in den letzten zwei bis drei Tagen) körperlich belasten können. Die Fragen beziehen sich auf Aktivitäten des täglichen Lebens, von denen Ihnen die meisten geläufig sein dürften.

Bitte beantworten Sie die Fragen auf der Grundlage Ihrer aktuellen Leistungsfähigkeit, also so wie Sie sich derzeit fühlen (das, was Sie sich derzeit zutrauen)!

FRAGE 2

Können Sie ein Stockwerk Treppen abwärts gehen, ohne anzuhalten?

$\square_{1} \mathrm{JA} \rightarrow$ weiter mit FRAGE $3 \quad \square_{0}$ NEIN $\rightarrow$ weiter mit FRAGE 5 (Fragen 3 und 4 entfallen!)

FRAGE 3

Können Sie WENIGSTENS EINE der folgenden Tätigkeiten ausführen?

- etwas mindestens 8 Treppenstufen hinauf tragen, ohne anzuhalten?

- leichte Gartenarbeiten verrichten (harken, Unkraut jäten)?

- ca. 6 Kilometer pro Stunde in der Ebene zügig spazierengehen?

- Tanzen?

- mit Ihrem Partner/Ihrer Partnerin intimen Verkehr haben, ohne dabei aufgrund körperlicher Beschwerden eine Pause zu machen?

$\square_{1}$ JA, ich kann eine oder mehrere dieser Tätigkeiten aus Frage 2 ausführen $\rightarrow$ weiter mit FRAGE 4

口 NEIN, keine dieser Tätigkeiten aus Frage 3 ist mir möglich $\rightarrow$ Ende dieses Bogens, weiter auf übernächster Seite (die Fragen 4-6 entfallen!) 
FRAGE 4

Können Sie WENIGSTENS EINE der folgenden Tätigkeiten ausführen?

- $10-12$ Kilogramm mindestens 8 Stufen hinauf tragen?

- einen Gegenstand heben und auf ebener Erde tragen, der ca. 35 Kilogramm wiegt?

- Schnee schaufeln bzw. Erde umgraben?

- Sport treiben (Ski fahren, Ballsportarten)?

- ca. $8 \mathrm{~km}$ pro Stunde sehr rasch gehen oder joggen?

$\square_{1}$ JA, ich kann eine oder mehrere dieser Tätigkeiten aus Frage 4 ausführen

$\rightarrow$ Ende dieses Bogens, weiter auf nächster Seite (Fragen 5 und 6 entfallen!) (I)

$\square_{0}$ NEIN, keine dieser Tätigkeiten aus Frage 4 ist mir möglich

$\rightarrow$ Ende dieses Bogens, weiter auf nächster Seite (Fragen 5 und 6 entfallen!) (II)

FRAGE 5

Können Sie WENIGSTENS EINE der folgenden Tätigkeiten ausführen?

- im Stehen duschen ohne Unterbrechung?

- sich entkleiden und das Bett machen?

- den Boden wischen?

- Wäsche aufhängen?

- Fenster putzen?

- 4 Kilometer pro Stunde langsam gehen)?

- Kegeln?

- Rasen mähen (mit Elektromäher)?

$\square_{1}$ JA, ich kann eine oder mehrere dieser Tätigkeiten aus Frage 5 ausführen

$\rightarrow$ Ende dieses Bogens, weiter auf nächster Seite (Frage 6 entfällt!)

$\square_{0}$ NEIN, keine dieser Tätigkeiten aus Frage 5 ist mir möglich $\rightarrow$ weiter mit FRAGE 6

\section{FRAGE 6}

Können Sie sich anziehen, ohne aufgrund von Luftnot oder Ermattung dabei eine Pause zu machen?

$\square_{1}$ JA $\rightarrow$ Ende dieses Bogens, weiter auf nächster Seite

$\square_{0}$ NEIN $\rightarrow$ Ende dieses Bogens, weiter auf nächster Seite 


\section{A.10 Verordnungsplan}

Derzeit nehme ich folgende Medikamente ein:

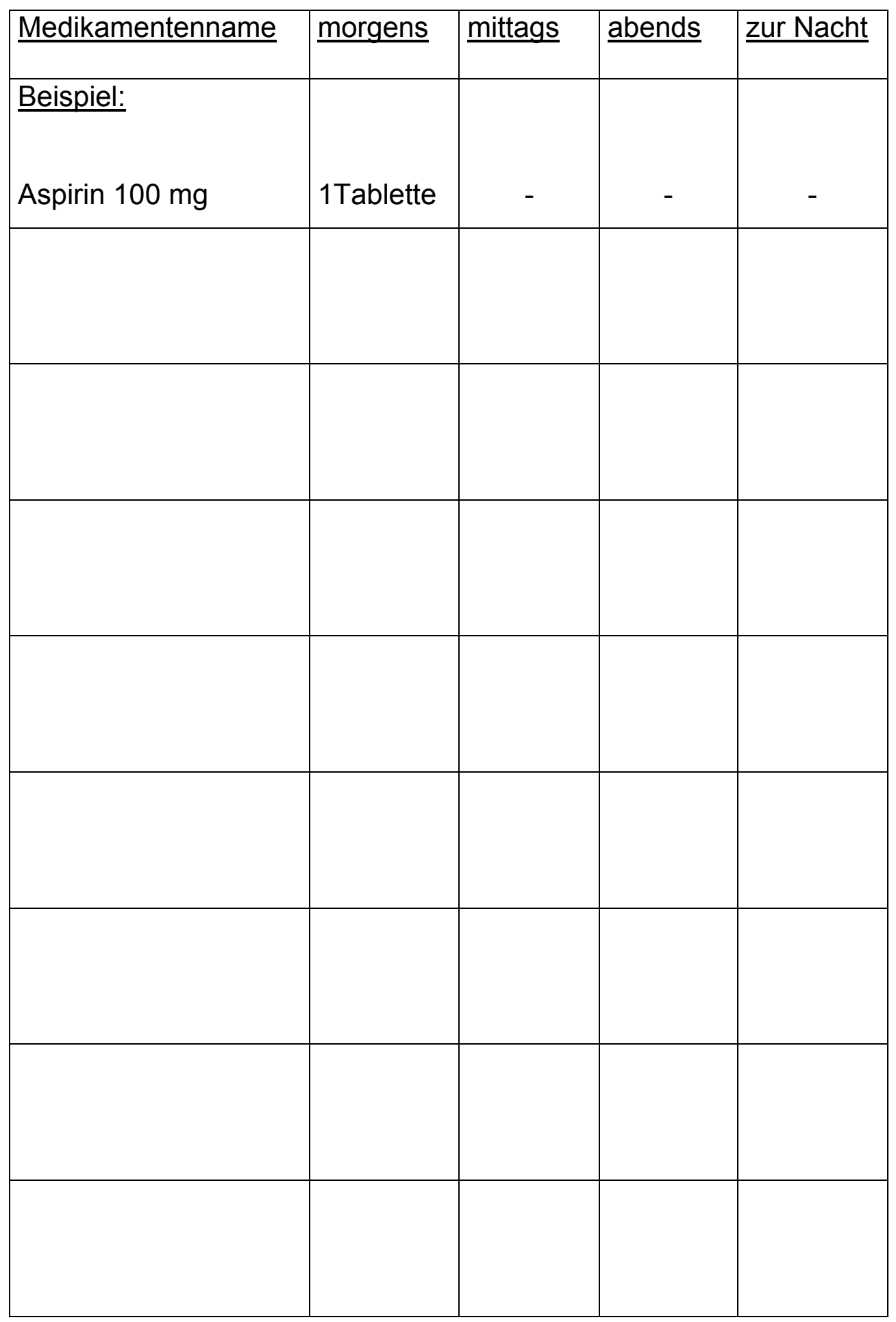




\section{Danksagung}

Ich danke Herrn Prof. Dr. Kochen, MPH, FRCGP für die freundliche Überlassung des Themas der Dissertation.

Mein besonderer Dank gilt Frau Dr. Janka Koschack für das überdurchschnittliche Engagement bei der Betreuung der Arbeit. Die stets motivierende und herzliche Atmosphäre sorgten für größten Arbeitseifer und weckten Interesse an weiterführenden Fragestellungen. Durch die Zusammenarbeit ist mir eine intellektuelle und große menschliche Bereicherung zuteil geworden.

Für die zahlreichen Anregungen und die konstruktive Kritik bei der Bearbeitung des Manuskripts danke ich Herrn Dr. med. Dieter Müller sowie meinen engen Freundinnen Lena Conradi und Jenny Weißflog.

Herrn Tobias Gohla danke ich für seinen Enthusiasmus und seine Geduld bei der Überwindung aller Hürden der Textverarbeitung auf dem Weg zur OnlineVeröffentlichung der Arbeit. 


\section{Lebenslauf}

Ich wurde am 27. Dezember 1978 als erster Sohn von Ulrike und Walter Pfeil in Rostock geboren. Am 7. Februar 1983 kam mein Bruder Johannes Pfeil zur Welt. Im September 1985 wurde ich eingeschult und besuchte bis zum Jahre 1992 zunächst die Grundschule und anschließend die Polytechnische Oberschule in Rostock. Anschließend wechselte ich bedingt durch die deutsche Wiedervereinigung an das Ahorn-Gymnasium, um nach einem erneuten Wechsel im Jahre 1995 an das JohannHeinrich-von-Thünen-Gymnasium mein Abitur zu absolvieren. Im Juli 1997 erlangte ich die Hochschulreife mit einem Notendurchschnitt von 2,4.

Im Anschluss daran absolvierte ich vom August 1997 an zunächst meinen zehnmonatigen Grundwehrdienst in Lüneburg, Torgelow und Schwerin, dem im Juni 1998 eine dreimonatige Ausbildung zum Unteroffizier der Reserve folgte.

Aufgrund meiner langjährigen Tätigkeiten als Jugendtrainer in Schwimm- und Basketballvereinen entschied ich mich im Oktober 1998, das Studium für das Lehramt am Gymnasium in den Fächern Sport und Englisch an der Universität Rostock zu beginnen.

Durch meine Erfahrungen und Erlebnisse im Rahmen einer Anstellung als studentische Hilfskraft im Fachbereich Sportmedizin sowie durch regelmäßige saisonale Einsätze im Wasserrettungsdienst am Strand von Rostock/Warnemünde wuchs der Wunsch, Medizin zu studieren. Im September 2000 wurde mir von der ZVS schließlich ein Studienplatz zugesprochen und somit begann ich mein Medizinstudium an der Georg-August-Universität in Göttingen zum Wintersemester 2000.

Nach der ärztlichen Vorprüfung 04/2002, die ich mit der Note 2,6 absolvierte, folgte ein Jahr später das erste Staatsexamen im September 2003 mit der Abschlussnote 3,0. Nach fünf weiteren Semestern konnte ich mich schließlich für das zweite Staatsexamen anmelden, welches ich ebenfalls mit der Note 2,6 abschloss. Anschließend verließ ich Göttingen, um mein praktisches Jahr am Pius-Hospital in Oldenburg und in Wanganui, Neuseeland zu bestreiten. Die Prüfungen des dritten Staatsexamens sind für den Zeitraum April bis Juni 2007 geplant.

Vor Beginn meines Medizinstudiums war ich ehrenamtlich als Jugendtrainer im Schwimmen und Basketball tätig. Bis zum Sommer 2004 arbeitete ich regelmäßig beim Wasserrettungsdienst des Deutschen Roten Kreuz in Rostock/Warnemünde 
und gab Kurse in erster und erweiterter erster Hilfe für Rettungsschwimmer, Führerscheinanwärter, Schüler sowie Sport- und Medizinstudenten. Im Rahmen des Lehramtstudiums war ich als wissenschaftliche Hilfskraft am Lehrstuhl für Biomechanik, Sportmotorik und Rehabilitation damit betraut, Versuche aufzubauen und zu leiten sowie Messungen durchzuführen und auszuwerten. Während des Medizinstudiums gab ich als Mitglied des studentischen Arbeitskreises Notfallmedizin (SAN) Medizinstudenten Kurse in Notfall-Basismaßnahmen (BLS) sowie in erweiterten ärztlichen Maßnahmen (ACLS). Darüber hinaus unterrichtete ich für die Johanniter-Unfallhilfe Kassel im Rahmen der Pflichtfortbildungen für Rettungsassistenten verschiedene Themen.

Seit Oktober 2004 arbeite ich als studentische Aushilfe auf der Intensivstation/Aufwachraum des evangelischen Krankenhauses Neu Bethlehem in Göttingen, wo ich viele praktische Erfahrungen sammeln konnte.

Durch eine zweimonatige Famulatur in Port Shepstone, Südafrika konnte ich erste Erfahrungen im nichteuropäischen Ausland sammeln, die mich sowohl fachlich wie auch menschlich sehr bereicherten.

Seit Mai 2004 bin ich als Doktorand in der Abteilung Allgemeinmedizin der Universität Göttingen unter Herrn Prof. Dr. Kochen mit einer Arbeit zur Qualität der medikamentösen Versorgung von Patienten mit Herzinsuffizienz durch den Hausarzt beschäftigt. 